\title{
Quality of service routing and mechanisms for improving video streaming over mobile wireless ad hoc networks
}

WILDER EDUARDO CASTELLANOS HERNÁNDEZ 



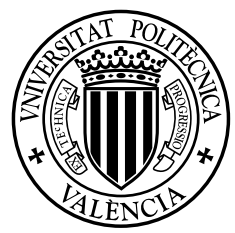

UNIVERSITAT POLITÈCNICA DE VALÈNCIA

DEPARTAMENTO DE COMUNICACIONES

\title{
Quality of Service Routing and Mechanisms for Improving Video Streaming over Mobile Wireless Ad hoc Networks
}

\author{
DOCTORAL THESIS \\ Wílder Eduardo Castellanos Hernández
}

Supervisor:

Dr. Juan Carlos Guerri Cebollada

Valencia, Spain

June - 2015 
Collection doctoral thesis

(c) Wilder Eduardo Castellanos Hernández

(c) 2015, of the present edition: Editorial Universitat Politècnica de València Telf.: 963877012 / www.lalibreria.upv.es

ISBN: 978-84-9048-428-9 (printed version)

Any unauthorized copying, distribution, marketing, editing, and in general any other exploitation, for whatever reason, of this piece of work or any part thereof, is strictly prohibited without the authors' expressed and written permission. 


\section{Abstract}

This thesis dissertation tackles the problem concerning provision of video streaming services over mobile wireless ad hoc networks. Such networks are characterized by their versatility and flexibility, features that make them particularly suited to be used in many scenarios. However, some limitations inherited of the wireless channel and the mobility of the nodes make difficult to guarantee certain degree of quality of service, which is a required condition to the multimedia applications. Furthermore, with the massive demand of video content, it has become very necessary for mobile ad hoc networks to have an efficient routing and quality of services mechanisms to support this traffic. This is because video streaming services require network to provide sufficient bandwidth and an upper bound in delay, jitter and loss rate. Consequently, traditional best-effort protocols are not adequate. The main contribution of this thesis is the development of a comprehensive routing protocol that has a feedback scheme in order to provide information to the application about the network conditions. This protocol has a crosslayer architecture and it incorporates three important enhancements. Firstly, a new route recovery strategy, which provides a mechanism to detect the link failures in a route and re-establish the connections taking into account the conditions of quality of service that have been established during the previous route discovery phase. Secondly, an algorithm for the estimation of the available bandwidth along the route, information that is sent to application layer in order to apply an adaptation procedure that adjusts the bit rate of the video source. This rate-adaptive strategy is performed exploiting the layered scheme of the scalable video coding. In particular, the adaptive method removes, from the scalable video stream, those layers that could not be efficiently supported by network since their bitrates exceed the available bandwidth. The third main feature of the proposed routing protocol is a gateway discovery algorithm to improve the interconnectivity between mobile ad hoc networks and infrastructurebased networks. This algorithm incorporates available bandwidth as a metric during the gateway selection and a dynamic adaptation of some operational parameters such as the size of the proactive area and the frequency of the advertisement messages.

Additionally, in order to solve the lack of a software tool to simulate rate-adaptive transmission of scalable video, a new simulation framework had be implemented. This simulation tool is an open source software freely available and, thus, it represents other contribution of this thesis. 
The results reveal performance improvements in terms of packet delay, dropped packets and the number of link failures while a more efficient use of the available bandwidth is obtained. In terms of video transmission, the results prove that the combined use of the proposed protocol and the scalable video coding provides an efficient platform for supporting rate-adaptive video streaming over mobile ad hoc networks. 


\section{Resumen}

Esta tesis aborda los problemas relacionados con los servicios de video en modo streaming sobre las redes móviles ad hoc. Este tipo de redes se caracterizan por su versatilidad y flexibilidad, lo cual las hace especialmente adecuadas para ser utilizadas en diversos escenarios. Sin embargo, algunas limitaciones inherentes a los enlaces inalámbricos y a la movilidad de los nodos, hace difícil garantizar cierto nivel de calidad de servicio, lo cual es una condición necesaria para el transporte de flujos multimedia. Además, con la masiva demanda de videos desde los dispositivos móviles, hace aún más necesario asegurar un encaminamiento eficiente y un cierto nivel de calidad de servicio en las redes móviles ad hoc. Por lo tanto, los tradicionales protocolos que funcionan bajo el modelo del "mejor esfuerzo" no son adecuados. Esto se debe principalmente a que las aplicaciones multimedia necesitan que la red asegure suficiente ancho de banda y unos valores máximos de retardo, jitter, y tasa de pérdidas.

La principal contribución de esta tesis es el desarrollo de un protocolo de encaminamiento que contiene un esquema de realimentación que le permite informar a la aplicación sobre las condiciones de la red. Este protocolo tiene una arquitectura cross-layer e incorpora tres importantes mejoras. Primero, una nueva estrategia de mantenimiento y recuperación de rutas que provee mecanismos para detectar los fallos de conectividad y el posterior re-establecimiento de las conexiones, teniendo en cuenta las condiciones de calidad de servicio que fueron establecidas durante la etapa inicial del descubrimiento de las rutas. Segundo, un algoritmo para la estimación del ancho de banda disponible a lo largo de la ruta, información que es enviada a la capa de aplicación para aplicar un proceso de adaptación que ajusta la tasa de envío de datos de la fuente. Esta estrategia adaptativa de la tasa aprovecha el esquema por capas de la codificación escalable de video. En particular, el método adaptativo elimina del flujo de video escalable, aquellas capas que no pueden ser transmitidas por la red ya que su tasa de bits supera el ancho de banda disponible. La tercera mejora incluida en el protocolo propuesto es un algoritmo de descubrimiento de gateways para mejorar la interconectividad entre las redes móviles ad hoc y las redes basadas en infraestructura. Dicho algoritmo utiliza el ancho de banda disponible para seleccionar el mejor gateway, así mismo, realiza una adaptación dinámica de algunos parámetros operacionales como el alcance y la frecuencia de los mensajes anuncio.

Adicionalmente, ha sido desarrollada una herramienta software para simular la transmisión adaptativa de video escalable sobre redes móviles ad hoc. Esta herramienta 
de simulación es un software de código abierto y constituye otra contribución más de esta tesis.

Los resultados muestran mejoras en el funcionamiento de las redes relacionadas con el retardo, la tasa de pérdidas de paquetes y el número de fallos en la conectividad. Simultáneamente, se obtiene un uso más eficiente del ancho de banda. En relación a la calidad del video transmitido, los resultados demuestran que la utilización del protocolo propuesto junto con la codificación de video escalable, provee un eficiente sistema para la transmisión adaptativa de video escalable sobre redes móviles ad hoc. 


\section{Resum}

Aquesta tesi aborda els problemes relacionats amb els serveis de vídeo en mode streaming sobre les xarxes mòbils ad hoc. Aquest tipus de xarxes es caracteritzen per la seva versatilitat i flexibilitat, la qual cosa les fa especialment adequades per a ser utilitzades en diversos escenaris. No obstant això, algunes limitacions inherents als enllaços sense fils i a la mobilitat dels nodes, fa difícil garantir cert nivell de qualitat de servei, cosa que és una condició necessària per al transport de fluxos multimèdia. A més, amb la massiva demanda de vídeos des dels dispositius mòbils, fa encara més necessari assegurar un encaminament eficient i un cert nivell de qualitat de servei en les xarxes mòbils ad hoc. Per tant, els tradicionals protocols que funcionen sota el model del "millor esforç" no són adequats. Això es deu principalment al fet que les aplicacions multimèdia necessiten que la xarxa asseguri suficient ample de banda i uns valors màxims de retard, jitter, i taxa de pèrdues.

La principal contribució d'aquesta tesi és el desenvolupament d'un protocol d'encaminament que conté un esquema de realimentació que li permet informar l'aplicació sobre les condicions de la xarxa. Aquest protocol té una arquitectura crosslayer i incorpora tres importants millores. Primer, una nova estratègia de manteniment i recuperació de rutes que proveeix mecanismes per detectar les fallades de connectivitat i el posterior re-establiment de les connexions, tenint en compte les condicions de qualitat de servei que van ser establertes durant l'etapa inicial del descobriment de les rutes. Segon, un algoritme per a l'estimació de l'ample de banda disponible al llarg de la ruta, informació que és enviada a la capa d'aplicació per aplicar un procés d'adaptació que ajusta la taxa d'enviament de dades de la font. Aquesta estratègia adaptativa de la taxa aprofita l'esquema per capes de la codificació escalable de vídeo. En particular, el mètode adaptatiu elimina del flux de vídeo escalable aquelles capes que no poden ser transmeses per la xarxa ja que la seva taxa de bits supera l'ample de banda disponible. La tercera millora inclosa en el protocol proposat és un algoritme de descobriment de gateways per millorar la interconnectivitat entre les xarxes mòbils ad hoc i les xarxes basades en infraestructura. Aquest algoritme utilitza l'ample de banda disponible per seleccionar el millor gateway, així mateix, realitza una adaptació dinàmica d'alguns paràmetres operacionals com l'abast i la freqüència dels missatges anunci. 
Addicionalment, ha estat desenvolupada una eina programari per a simular la transmissió adaptativa de vídeo escalable sobre xarxes mòbils ad hoc. Aquesta eina de simulació és un programari de codi obert i constitueix una altra contribució més d'aquesta tesi.

Els resultats mostren millores en el funcionament de les xarxes relacionades amb el retard, la taxa de pèrdues de paquets i el nombre de fallades en la connectivitat. Simultàniament, se n'obté un ús més eficient de l'ample de banda. En relació a la qualitat del vídeo transmès, els resultats demostren que la utilització del protocol proposat juntament amb la codificació de vídeo escalable, proveeix un eficient sistema per a la transmissió adaptativa de vídeo escalable sobre xarxes mòbils ad hoc. 


\section{Acknowledgments}

I would like express my deepest gratitude to my supervisor Dr. Juan Carlos Guerri for his excellent guidance, continuous support and encouragement throughout my Ph.D. study. This thesis dissertation would have not been possible without his help.

I owe my thanks to all my colleagues in the Multimedia Communications Group for their help. Specially, I would like to thank Dr. Pau Arce for reading my papers and providing me insightful comments and valuable ideas.

It also has been a great pleasure to work with my co-workers of the Biomechanical Institute of Valencia. They gave me an essential background and many useful tools for my research career.

I am also very grateful for all those friends who helped me during difficult moments and with whom I shared many happy moments. Without their generous help, I would not have adapted so well to my life in Spain. I would like to thank Andreea for helping me during the writing of the papers. I am sure she will never forget the new and wonderful words she discovered.

My special thanks go to Mónica. Without her support and love, I could not accomplish this work. She has given me much more than I could imagine.

Finally, I would like to thank my parents, as well as my sisters, for everything they have given me; they have always been supportive for everything I have done. 

The most profound technologies are those that disappear. They weave themselves into the fabric of everyday life until they are indistinguishable from it

$\sim$ Mark Weiser 

To my parents and Monica... 



\section{Contents}

Chapter 1-Introduction ............................................................................................................ 1

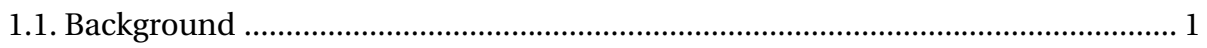

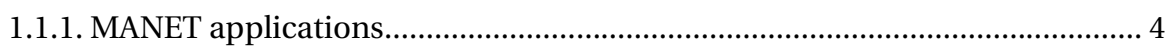

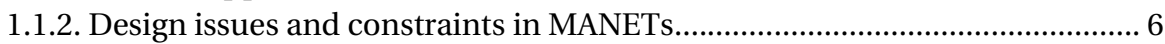

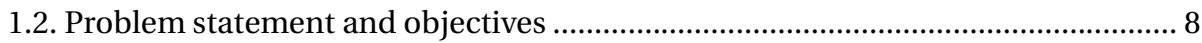

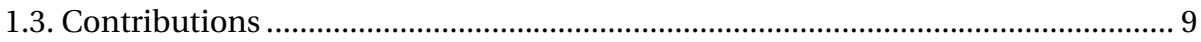

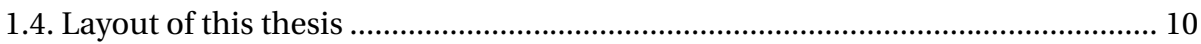

Chapter 2-Introduction to Video Streaming over MANETs......................................... 13

2.1. Achievable transmission rate in wireless ad hoc networks.................................. 13

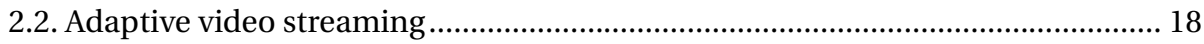

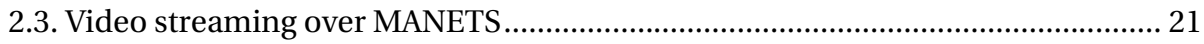

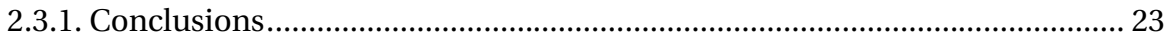

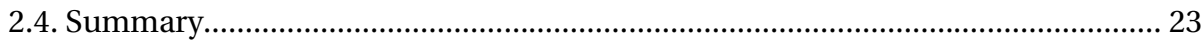

Chapter 3-AQA-AODV: A cross-layer routing Protocol for MANETs.........................25

3.1. An overview on routing protocols in mobile ad hoc networks: ............................ 25

3.2. QoS routing protocols for mobile ad hoc networks............................................ 27

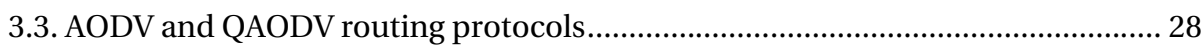

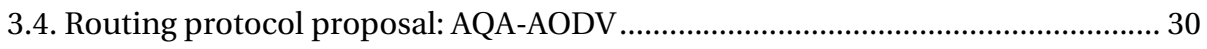

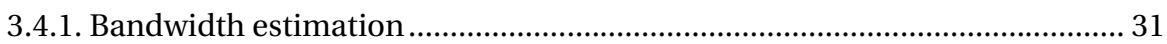

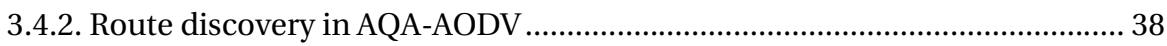

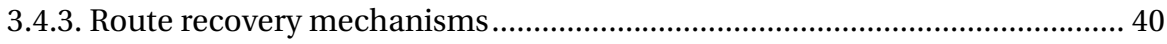

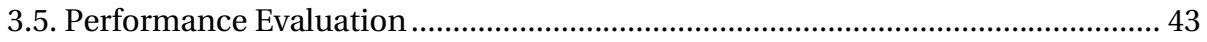

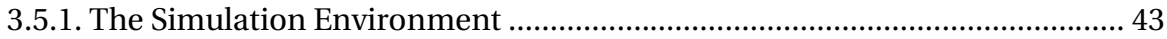

3.5.2. Sample Network: static linear topology with 7 nodes .................................... 44

3.5.3. Scenario 2: Static linear topology with variable length .................................... 46

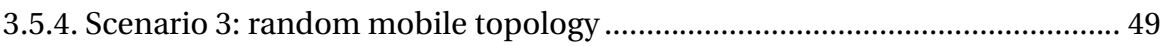

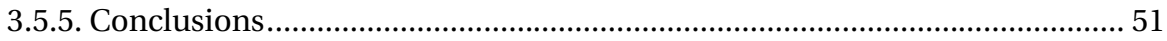




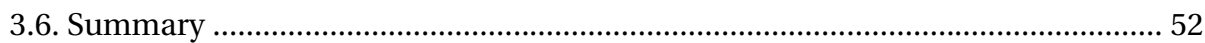

Chapter 4-Adaptive Video Streaming over MANETs using AQA-AODV.................... 53

4.1. Adaptive scalable video streaming ....................................................................... 53

4.1.1. Scalable Video Coding .................................................................................... 54

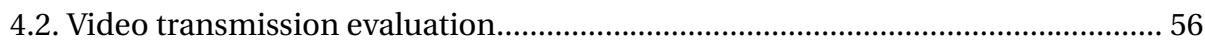

4.3. Proposed evaluation framework for scalable video streaming............................. 58

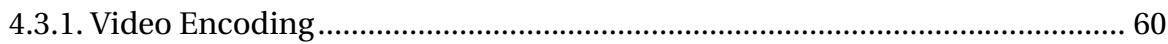

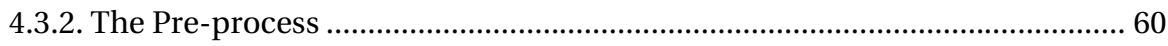

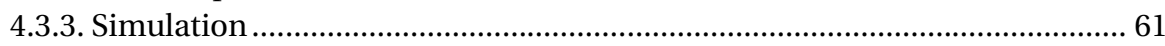

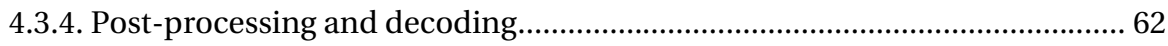

4.4. Performance analysis of the SVCEval-RA framework.......................................... 63

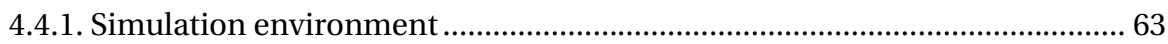

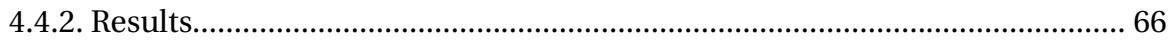

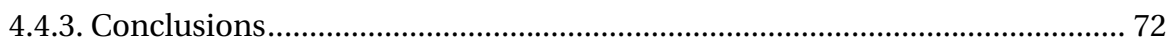

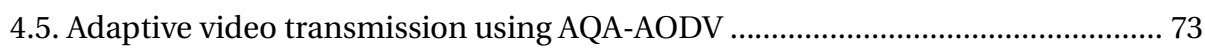

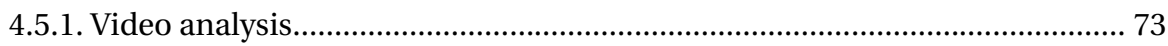

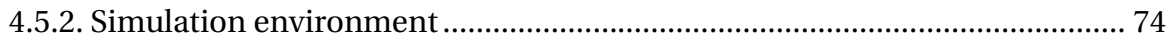

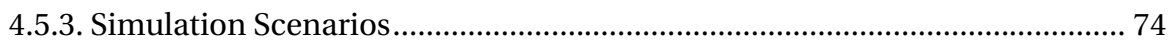

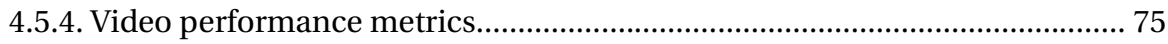

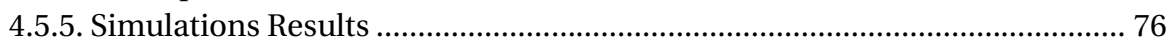

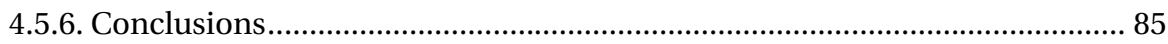

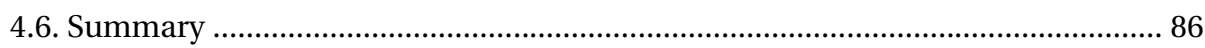

Chapter 5-QoS-aware gateway discovery extension for AQA-AODV ........................ 87

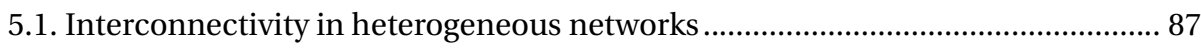

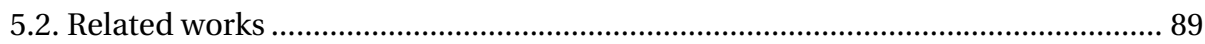

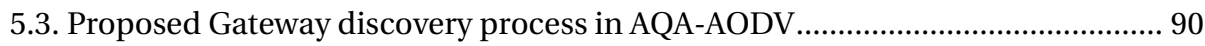

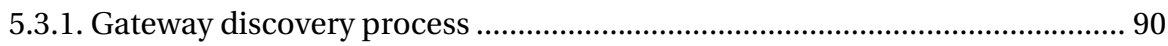

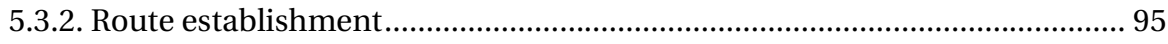

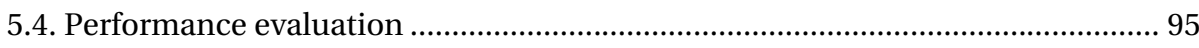

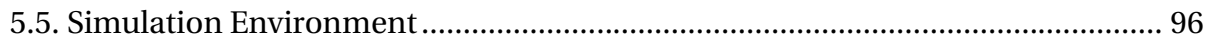

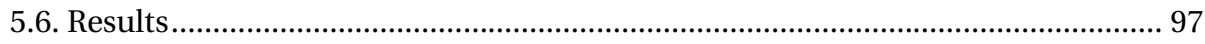

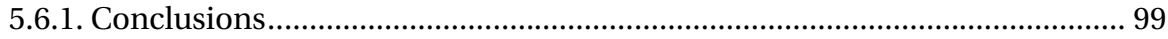

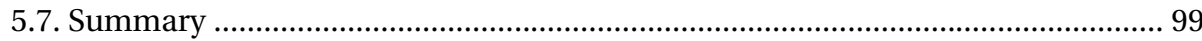

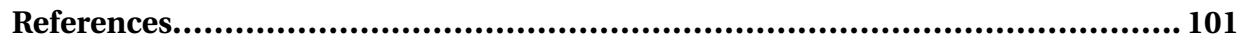

Appendix A-List of Publications ............................................... 109 


\section{List of Tables}

Table 3.1. QoS routing solutions - Capability and prerequisites....................................................29

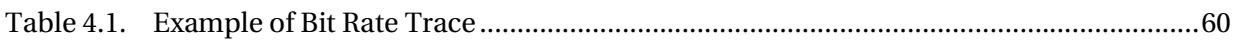

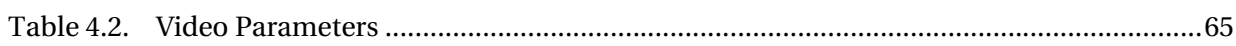

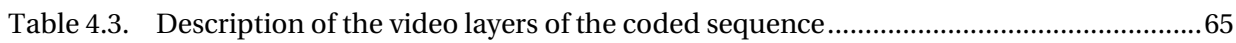

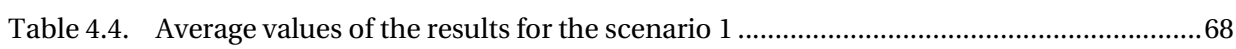

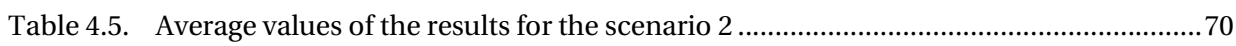

Table 4.6. Average values of the results for the scenario 3........................................................... 72

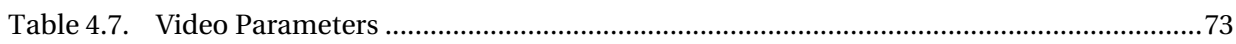

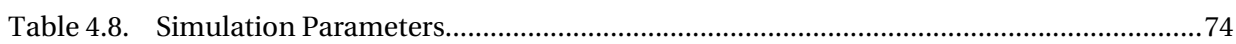

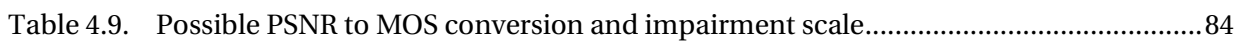

Table 5.1. Information for the calculation of the network stability factor ......................................94

Table 5.2. Values for the estimation of the advertisement interval .............................................95

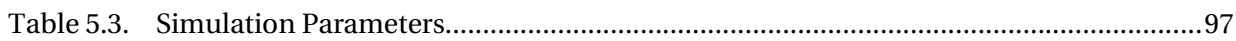





\section{List of Figures}

Figure 1.1. Conceptual representation of a mobile ad hoc network................................................

Figure 1.2. MANET extending Internet services to a non-infrastructure area ...................................

Figure 1.3. MANET layered stack proposed by IETF MANET working group [1] ...............................

Figure 2.1. Intra-flow contention and MAC interference in a chain of nodes...................................14

Figure 2.2. Main simulation parameters and network scenario ..................................................... 15

Figure 2.3. Average throughput achieved in the chain, as a function of the chain length in wireless ad hoc networks for 802.11b@2Mbps (a), 802.11b@11Mbps (b), 802.11g@54Mbps (c)

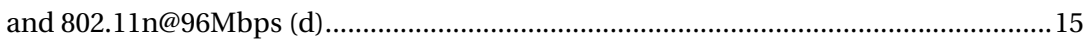

Figure 2.4. Percentage of lost packet as a function of number of nodes ........................................... 16

Figure 2.5. Average throughput vs. offered load in a 7-node chain network for 802.11b@2Mbps (a), 802.11b@11Mbps (b), 802.11g@54Mbps (c), 802.11n@96Mbps (d)...............................17

Figure 2.6. Percentage of Lost Packets for 802.11 mode................................................................... 18

Figure 2.7. Sending rate per node in a chain of 7 nodes ............................................................... 18

Figure 2.8. Traditional video streaming.................................................................................... 19

Figure 2.9. Stream switching technique ...................................................................................... 19

Figure 2.10. Comparison of the scalable video streaming (a) and the adaptive scalable video streaming (b) ......................................................................................................2

Figure 2.11.Video quality degradation in multi-hop wireless networks ...........................................21

Figure 2.12. Packet Losses (a), chain throughput (b), packet delay (c) and PSNR (d), as a function of

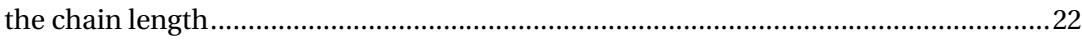

Figure 3.1. Routing protocols for mobile ad hoc networks: approaches and classification ............26

Figure 3.2. Functional block diagram of AQA-AODV......................................................................

Figure 3.3. Example of wireless ad hoc network with the available bandwidth of each link...........32

Figure 3.4. Flowchart of the tasks involved in the bandwidth estimation phase ..............................33

Figure 3.5. Transmission of a single packet in IEEE 802.11 DCF MAC protocol ...............................34

Figure 3.6. Using HELLOs packets in the bandwidth estimation .....................................................

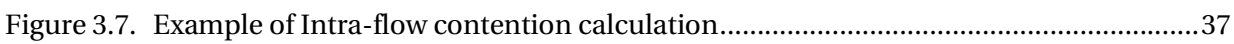

Figure 3.8. Example of the route discovery phase in AQA-AODV .....................................................39

Figure 3.9. Example of route recovery mechanism. Case 1 ............................................................. 41

Figure 3.10. Example of route recovery mechanism. Case 2 .......................................................... 42 


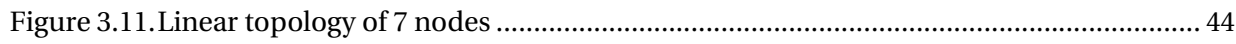

Figure 3.12.Throughput delivered by a 7 -node chain with different send rate ................................ 44

Figure 3.13.Packet loss in 7-node chain with different transmission rate ......................................... 45

Figure 3.14.Average end-to-end delay in 7-node chain with different transmission rate................ 46

Figure 3.15. Number of link failures (a) and Connection Setup Latency (b) in linear scenario of 7

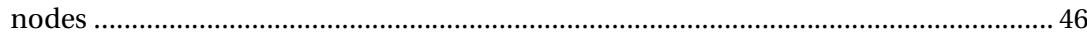

Figure 3.16. Scenario 2: static linear topology with variable length. The transmission and

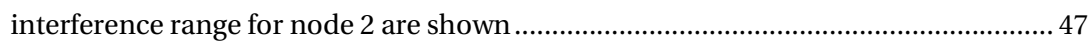

Figure 3.17. Throughput achieved along the path as a function of the chain length ......................... 47

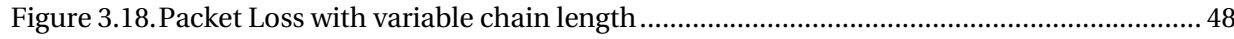

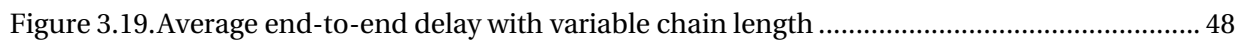

Figure 3.20. Number of link failures and Connection Setup Latency (CLS) vs. number of nodes... 49

Figure 3.21.Packet Loss with variable requested rate..................................................................... 50

Figure 3.22.Average end-to-end delay with variable requested rate............................................... 50

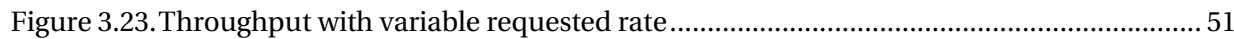

Figure 3.24.Evaluation results of the route recovery mechanism: (a) Number of link failures and (b) CSL (Connection Setup Latency) as a function of the requested rate............................ 51

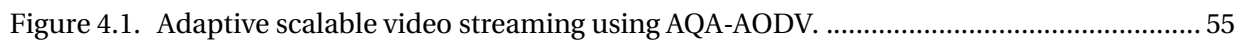

Figure 4.2. Example of coding structure of a SVC stream with temporal and quality scalability .. 56

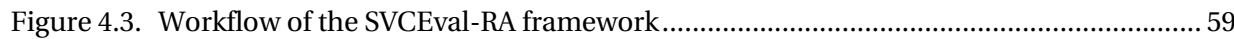

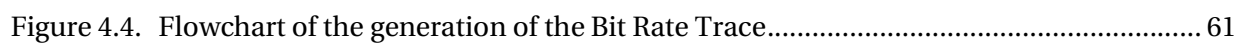

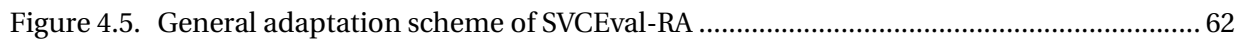

Figure 4.6. Network scenarios: Scenario 1: Persistent variations of the available bandwidth (a), Scenario 2: "Positive" peaks in the available bandwidth (b) and Scenario 3: "Negative" peaks in the available bandwidth (c) . .64

Figure 4.7. Description of the video layers (a) and Video properties used in the simulation study (b) 66

Figure 4.8. Throughput of the video flows (a), Lost Packet Rate (b), and Y-PSNR (c) for Scenario1 67

Figure 4.9. SAD estimation of the test sequence. 69

Figure 4.10. Throughput of the video flows (a), Lost Packet Rate (b), and Y-PSNR (c) for Scenario2 ("Positive" peaks in the available bandwidth).

Figure 4.11.Throughput of the video flows (a), Lost Packet Rate (b), and Y-PSNR(c) for Scenario 3 ("Negative" peaks in the available bandwidth) 71

Figure 4.12.Description of the SVC layers contained in the video stream (a) and Rate-distortion analysis of the SVC video stream (b) 74

Figure 4.13.Scenario 1: "roaming node". Video Source is node 0 and the destination is node 1 ..... 75

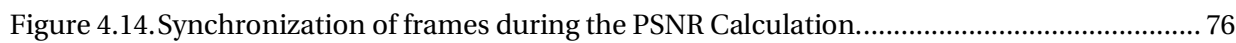

Figure 4.15.Available Bandwidth and Throughput achieved during simulation as a function of the

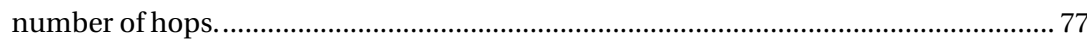

Figure 4.16. Packet Loss as a function of the route length .................................................................. 77 xviii 
Figure 4.17.Average end-to-end delay during the network simulation ..............................................78

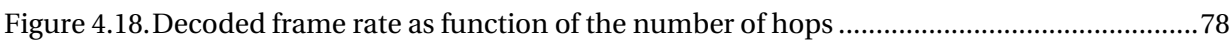

Figure 4.19.Average Y-PSNR as function of the number of hops ........................................................79

Figure 4.20. Packet Loss for a mobile scenario with different pause times ......................................... 81

Figure 4.21.Average end-to-end delay as function of pause time....................................................

Figure 4.22.Decoded Frame Rate as a function of the pause time..................................................... 82

Figure 4.23.Average Y-PSNR as a function of the pause time......................................................... 82

Figure 4.24. Y-PSNR and Bandwidth Available as a function of the frame index (mobile topology, pause time $=80 \mathrm{~s}$ ). Results from frame 0 to 2250 (a) and from frame 2251 to 2506 (b) 83

Figure 4.25.Cumulative Distribution Function (CDF) of PSNR per frame (mobile topology, pause

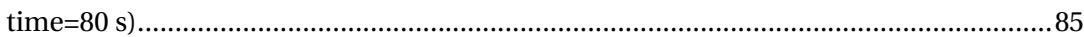

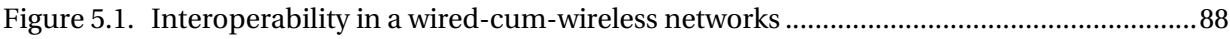

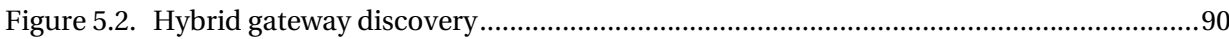

Figure 5.3. Message sequence in gateway selection procedure .......................................................

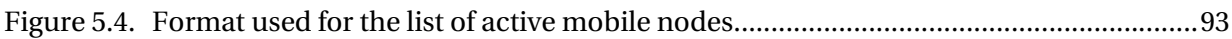

Figure 5.5. Relationship between NSF and the advertisement time..............................................

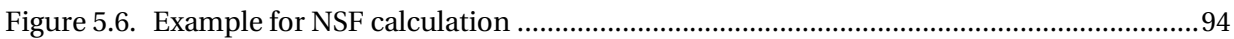

Figure 5.7. Variation of the Network Stability Factor....................................................................95

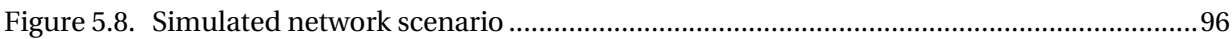

Figure 5.9. Packet Loss with variable node speed in both scenarios: (a) 10 sources and (b) 15

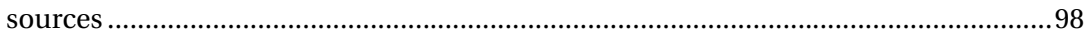

Figure 5.10. Delay as a function of the node speed in both scenarios: (a) 10 sources and (b) 15

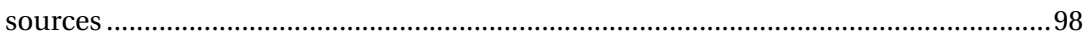

Figure 5.11. Routing overhead with variable node speed in both scenarios: (a) 10 sources and (b) 15 sources 99 



\section{Acronyms}

$\begin{array}{ll}\text { 4G } & \text { 4th Generation } \\ \text { ACK } & \text { Acknowledgement } \\ \text { AODV } & \text { Ad hoc On-demand Distance Vector } \\ \text { AQA-AODV } & \text { Adaptive QoS-Aware AODV protocol } \\ \text { ARQ } & \text { Automatic Repeat Request } \\ \text { BWav } & \text { Available Bandwidth } \\ \text { BWconsumed } & \text { Consumed Bandwidth } \\ \text { CBR } & \text { Constant Bit Rate } \\ \text { CCmax } & \text { Maximum Contention Count } \\ \text { CIF } & \text { Common Intermediate Format } \\ \text { CSL } & \text { Connection Setup Latency } \\ \text { CSMA/CA } & \text { Carrier Sense Multiple Access with Collision Avoidance } \\ \text { DCCP } & \text { Datagram Congestion Control Protocol } \\ \text { DSR } & \text { Dynamic Source Routing } \\ \text { FPS } & \text { Frames Per Second } \\ \text { GoP } & \text { Group of Pictures } \\ \text { GWADV } & \text { Gateway Advertisement message } \\ \text { GWREQ } & \text { Gateway Request message } \\ \text { HTTP } & \text { Hypertext Transfer Protocol } \\ \text { IEEE } & \text { International Electrical and Electronic Engineers } \\ \text { IETF } & \text { Internet Engineering Task Force } \\ \text { IP } & \text { Internet Protocol } \\ \text { IR } & \text { Interference Range } \\ \text { ITU } & \text { International Telecommunication Union } \\ \text { MAC } & \text { Media Access Control } \\ \text { MANET } & \text { Mobile Ad hoc Network } \\ \text { MOS } & \text { Mean Opinion Score } \\ \text { NSF } & \text { Network Stability Factor } \\ \text { NS2 } & \text { Network Simulator 2 } \\ \text { PAN } & \text { Personal Area Network } \\ \text { PoB } & \text { Play-out Buffer } \\ \text { PSNR } & \text { Qeak Noise to Signal Ratio } \\ \text { QAODV } & \text { Quality of Experience } \\ \text { QoE } & \end{array}$




$\begin{array}{ll}\text { QoS } & \text { Quality of Service } \\ \text { reqBW } & \text { Requested Bandwidth } \\ \text { RREP } & \text { Route Reply Packet } \\ \text { RREQ } & \text { Route Request Packet } \\ \text { RRER } & \text { Route Error Packet } \\ \text { RTS/CTS } & \text { Request to Send/Clear to Send } \\ \text { sid } & \text { Session Identifier } \\ \text { SNR } & \text { Signal to Noise Ratio } \\ \text { SVC } & \text { Scalable Video Coding } \\ \text { TCP } & \text { Transmission Control Protocol } \\ \text { Thpacket } & \text { Packet throughput } \\ \text { TTL } & \text { Time To Live } \\ \text { UDP } & \text { User Datagram Protocol } \\ \text { VANET } & \text { Vehicular Ad hoc Network } \\ \text { VoD } & \text { Video on Demand } \\ \text { WMN } & \text { Wireless Mesh Networks } \\ \text { WSN } & \text { Wireless Sensor Networks }\end{array}$




\section{Chapter 1}

\section{Introduction}

This chapter offers an introduction to the background of this thesis and gives an overview of the fundamental concepts of wireless ad hoc networks, their main features, possible application scenarios and limitations. In addition, the objectives and the organization of this thesis are also described, as well as the most relevant contributions addressing adaptive video streaming over mobile ad hoc networks.

\subsection{Background}

At the end of the 1990s, The IEEE 802.11 subcommittee adopted the term "ad hoc model" to denote a new communication paradigm in which nearby nodes can communicate directly by exploiting wireless networks technologies as Zigbee (IEEE 802.15.4), Bluetooth (IEEE 802.15.1), 802.11(WI-FI) or 802.16 (WiMAX). The first commercial applications of the ad hoc technology allow network devices to establishment a singlehop ad hoc network, which are the simplest form of infrastructureless networks or selforganizing networks, by interconnecting devices that are within the same transmission range. However, soon emerged the multihop network paradigm, often referred to as Mobile Ad hoc Networks (MANETs), which was conceived to extend the possibility to information exchange with any wireless node, without the need to develop any network infrastructure. In MANETs, nearby users directly communicate (setting their wireless network interfaces of their devices in ad hoc mode) not only to exchange their data but also to relay the traffic of other network nodes that cannot directly communicate. For this reason, in a MANET, wireless devices must cooperatively provide the functionality that is usually provided by the network infrastructure (e.g., routers, switches, servers). Figure 1.1 provides a conceptual representation of a MANET. In many situations such as catastrophes or emergencies in which there are no infrastructures or they cannot be installed because of geographical or temporal reasons, this kind of network may be an ideal solution.

In addition, a mobile ad hoc network not only works in isolated mode but also it can be connected to a larger network scenario like the one shown in Figure 1.2 [1]. 


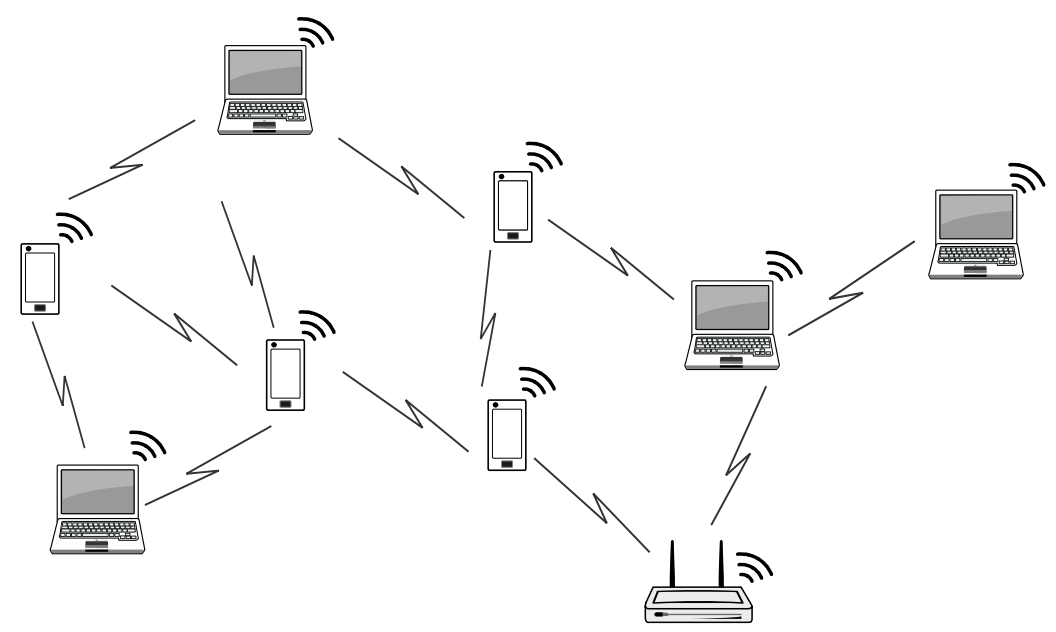

Figure 1.1. Conceptual representation of a mobile ad hoc network

With the advent of newer network architectures, new scenarios are emerging as applications of MANETs such as Smart cities and IoT (Internet of Things). In this sense, mobile ad hoc networks are becoming an integral part of next-generation networks (4G) because of its flexibility, autoconfiguration capability, lack of infrastructure, ease of maintenance, self-administration capabilities and cost-effectiveness.

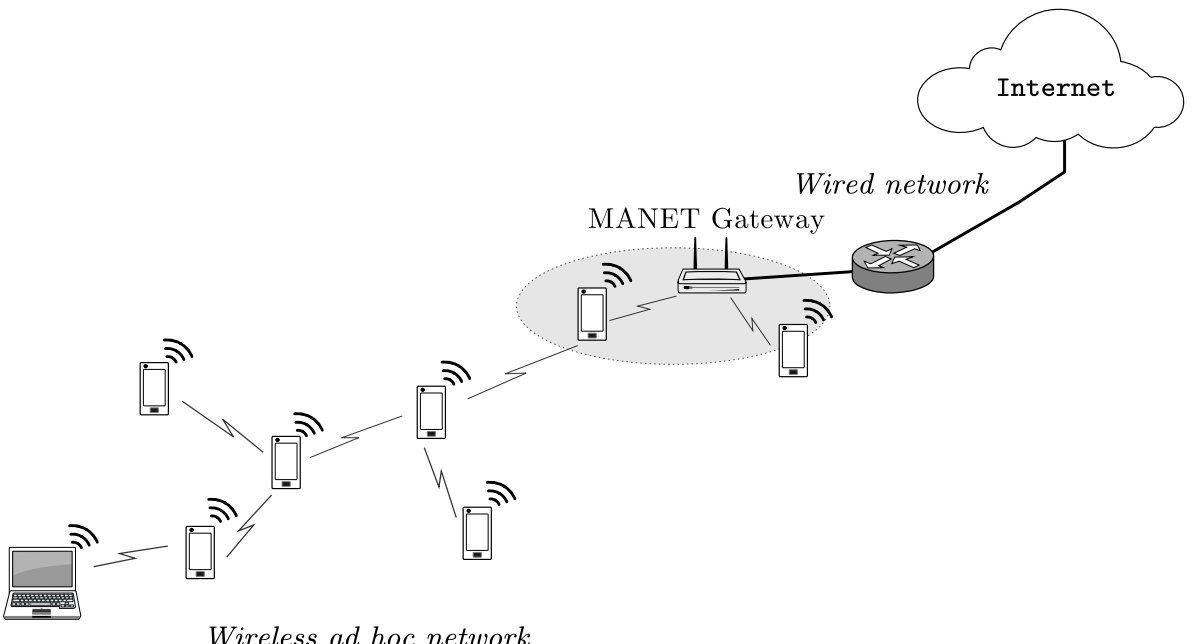

Wireless ad hoc network

Figure 1.2. MANET extending Internet services to a non-infrastructure area

In this context, the mobile ad hoc networks have been an intense research theme in the academic community during the last two decades. This intense research activity is reflected in the fact that approximately 6278 articles have been published in scientific 
journals in the area of MANETs since $1995^{1}$. Approximately $32 \%$ of these publications (1982 articles) were published in the last three years (about 660 articles per year), corresponding to the years with the highest number of published articles. This fact illustrates that MANETs is still a topic of great interest for scientific research.

Taking as reference the MANET protocol stack of the MANET IETF working group (see Figure 1.3), all layers have been subject of research activities. Some enhancements at the physical layer included the use of directional antennas, improved signal processing schemes, software defined radio, and mesh technologies; while at the MAC layer there have been several proposals for collision control and efficient energy consumption. However, most research works have been mainly focused on the network layer [2]-[7] while higher layers are the areas in MANETs theme where less have been performed research activities [1].

One of the most important force that have driven the research activities in MANETs are the multimedia applications. At recent years, video streaming over MANETs is a very fascinating research topic, mainly due to the high popularity of mobile devices, such as notebooks, smartphones, and tablets. Additionally, the computing capabilities of these devices are growing quickly and, at the same time, the demand of multimedia content from mobile devices is becoming increasingly widespread.

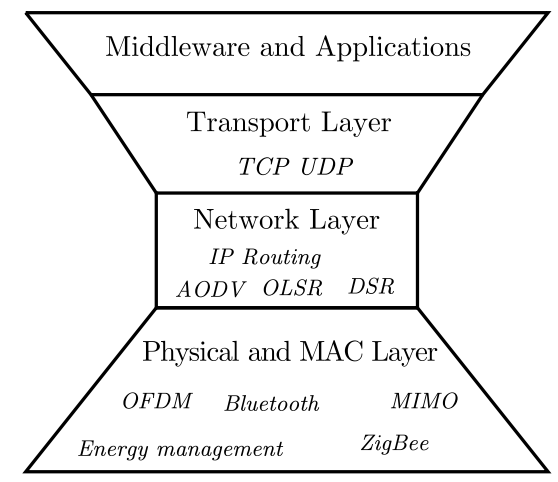

Figure 1.3. MANET layered stack proposed by IETF MANET working group [1]

Supporting video streaming services in mobile ad hoc networks presents many challenges due to the difficulty of providing certain quality in the shared wireless medium and the dynamic topology of MANETs [8]. These issues impose the necessity of developing new routing protocols with the aim of achieving efficient routes between the nodes where information is available in destination nodes reliably and within boundary time. Therefore, routing protocols should have a good performance in terms of overhead, a route convergence and bandwidth consumption, even when there are

\footnotetext{
1 Data obtained from the Scopus database by searching for keywords in article titles and abstracts related to specific topic of MANETs and discarding terms as wireless ad hoc sensor networks, cellular networks, vehicular ad hoc networks and cognitive networks.
} 
changes in traffic load or the number of nodes. Besides routing protocols, video delivery can be improved through cross-layer techniques since some functions cannot be assigned to a single layer (e.g. energy control and cooperation). Practical cross-layer solutions may try to improve communications gathering information from several layers about routing or network state in order to allow multimedia applications to adapt bitrates or even request recent lost packets [9]. This kind of technique could be very versatile to handle situations with network congestion or important video degradation caused by node mobility. Therefore, despite the complexity of providing hard Quality of Service (QoS) for multimedia applications over MANETs, there are still many options to improve video streaming quality, through holistic approaches that involve routing, transport and application layers.

\subsubsection{MANET applications}

Although initially mobile ad hoc networks were conceived as a general-purpose network, in terms of real-world deployment and industrial adoption, MANET applications are envisaged as specialized networks that are managed by a single authority and tailored to solve specific problems. For example in military networks, vehicular networks or sensor networks. Additionally, MANETs are expected to become a key component in the $4 \mathrm{G}$ architecture. Similarly, ad hoc networking technique is expected to become an important functionality of overall next-generation wireless network technologies.

In this context, typical applications and some of the most illustrative use cases nowadays are described below:

- $\quad$ Tactical networks

MANETs are appropriate to support communication and coordination needs between soldiers, military vehicles and information headquarters. The advantages that offer MANETs in military applications are manifold. Firstly, their distributed architecture decrease the risk of communication failure since do not exist a fixed infrastructure can be attacked. Secondly, tactical networks based on MANETs can be rapidly deployable even in areas where no infrastructure is available.

- $\quad$ Extended network connectivity

MANETs also can be used to provide communication between devices or with the Internet in areas with limited infrastructure or intermittent access. Two examples are the Interplanetary Internet and the Rural Internet system. The first one, is a multihop communication system between devices as satellites, shuttles, and surface stations with the aim of provide a solution to the extreme distances when a single-hop communication is used. The Rural Internet is a networking system that extend the Internet connectivity to remote areas through electronics devices, both personal (e.g. mobile phones) and community-owned. Some examples of this application are the Kiosknet system [10], the Sustainable Access in Rural India (SARI) [11] and the Saami Network Connectivity in the artic Sweden [12] 


\section{- Emergency Services}

Ad hoc networks have traditionally been used in disaster situations after disasters or catastrophes, such as flood, earthquake or fire. Ad hoc networks not only provide communication between the emergency teams, but also they can be used to rapidly deploy self-organized devices such as unmanned aerial vehicles (UAVs) or autonomous robots, so as to minimize response time and maximize the efficiency of available resources. An example of an emergency ad hoc network is STIGMERGY [13], which is a network of stationary sensor nodes (also referred to as tags) that can be established directly on the emergency field.

\section{- Vehicular Networks (VANETs)}

The novel Intelligent Transportation Systems involve a particular case of ad hoc networks known as Vehicular Networks (VANETs). In these networks, vehicles are equipped with wireless interfaces that enable them to communicate with each other (V2V) or with road-side fixed infrastructure (V2I). On the one hand, V2V communications allow vehicles to participate in vehicle coordination platforms as well as the routing of other communications and, on the other hand, V2I connectivity allow vehicles to obtain information about road conditions, traffic congestion or accident warnings, and at the same time, control platforms can update traffic lights in real time in order to optimize traffic flow in case of congestion. Actually, IEEE is working in the family of standards IEEE 1609 [14] to define an architecture and a complementary, standardized set of services and interfaces that collectively enable secure V2V and V2I wireless communications.

- Wireless Personal Area Networks (PANs)

This kind of ad hoc networks allows the proximal electronic devices with specific purposes, such as cameras, storage devices, televisions, mobile phones, or laptops, to dynamically share information through an autonomous home networks.

\section{- $\quad$ Body Area Networks (BANs)}

There are some situations where carrying and holding a computer or laptop are not practical, for example, in an assembly line or working at height. In these cases, a wearable computer can provide a solution for ubiquitous computing by distributing computer components (e.g. head-mounted, processors, mass storage, displays, earphones and microphones) on the body [15], [16]. Similarly, a system of wireless medical sensors can be located in or around a human body operating as a health monitoring system, which can be used in a wide range of applications [17], such as remote health/fitness monitoring, rehabilitation, disease prevention or for sports training. Some examples of BANs formed by medical sensors used in telemedicine systems can be found in [18], [19].

- Wireless Sensor Networks (WSNs)

Besides BANs, PANs, VANETs and military networks, MANETS also can be used to interconnect a set of low-cost and low-power sensor devices deployed in the 
environment or, alternatively, carried by animals. These devices usually are embedded, for instance, in buildings, bridges, streets, on animals, or mountains and they are used to environmental or industrial monitoring and, more generally, to monitor events and phenomena. Bennett et al. [20] Stajano et al. and [21] give some examples of the application of WSNs in civil engineering monitoring.

\section{- $\quad$ Smart Cities}

The recently proposed model of an intelligent management of the available resources in a city, known as "Smart Cities", emerges as other application of the wireless ad hoc networking [22]. Smart Cities features the utilization of a wireless communication system in order to interconnect the smart devices deployed in the city and facilitate the content dissemination of between users. New services and applications can be provided from wireless sensors and actuators for control and coordination of physical services (such as adaptive traffic lighting, waste management and smart signalling) [23]-[27] as well as to learn the behaviours and needs of the citizens and adapt the services of the city.

- Internet of Things (IoT)

IoT is a novel communication paradigm that outlines a new scenario of the modern wireless communication where all the devices communicate via a common platform and thus can easily be controlled remotely [28], [29]. The main goal is to construct a virtual platform where the objects or things (such as a sensor network, vehicle, appliance, mobile phones, etc.) are able to interact with each other and they can freely participate and offer/receive any type of service.

\subsubsection{Design issues and constraints in MANETs}

Although a wireless ad hoc network have many advantages, such as versatility, selforganization, self-administration, ease of deployment, and so on; these benefits have a price. Wireless ad hoc networks get the conventional problems of wireless communications, such as bandwidth limitation, energy constrains and limited quality. In addition, the mobility of the networks nodes, multihop nature, and the lack of fixed infrastructure create a number of difficulties and design constraints that are new to mobile ad hoc networks, as discussed below:

- Infrastructureless

The lack of fixed infrastructure of mobile ad hoc networks generates new design issues compared with fixed networks. In addition, lack of a centralized administrator means network management has to be distributed across different nodes, which brings added difficulty in fault detection and management.

- Dynamic network topology

In mobile ad hoc networks, network topology may change frequently and unpredictably since nodes can move arbitrarily. This fact causes route changes, frequent connectivity failures and packet losses. 
The network topology in a wireless ad hoc network is highly dynamic due to the mobility of nodes. Since nodes may move in and out of coverage of each other, topology may change rapidly and unpredictably, and connectivity among devices may vary with time.

- $\quad$ Resource constrained operation

Generally, mobile nodes in a MANET, such as smartphones, sensors or portable devices, have low or moderate processing capabilities, memory size and limited power. This resource-constrained situation causes a low-performance operation with a limited storage. Even though new cutting-edge mobile devices are emerging nowadays more powerful and efficient, energy efficiency still seems insufficient because computing functions and routing services become more complex. In this context, transmission capabilities and the applications that can be supported by each mobile node are also reduced.

- $\quad$ Limited link capacity and quality

Because wireless physical medium is shared among nodes, only a transmitting node within range can use wireless channel. This fact leads to low efficient in the bandwidth use and a variable capacity of the links due to mutual interferences.

- Network connectivity and reliability

Network connectivity in MANETs is obtained by routing and forwarding among multiple nodes. This fact introduces two main problems. Firstly, the route discovery process is assumed by resource-limited nodes, which must execute a routing protocol to find suitable routes. Secondly, when a node fails finding a route or forwarding a packet the lack of centralized monitoring and a management entity leads to these types of misbehaviours cannot be detected quickly, adding substantial complexity to protocol design.

- Quality of Service

Certain level of quality of service (QoS) is essential for successful delivery of multimedia content. Achieving certain QoS requirements (such as throughput, delay, packet loss, jitter, error rate) are very difficult in MANETs because of the network characteristics and constraints described above, such as dynamically network topology, limited link capacity and node capabilities.

- Network Security

Mobile wireless networks are generally more vulnerable to information and physical security threats than fixed-wireline networks. The use of open and shared broadcast wireless channels makes the nodes in a mobile ad hoc network more vulnerable to security threats. Moreover, without a centralized infrastructure the security control must be distributed to each node, which is harder to implement. 


\subsection{Problem statement and objectives}

Despite MANETs are very versatile and appropriate to be used in many scenarios due to the infrastructure-less and self-organized characteristics, this kind of networks have important limitations, such as bandwidth-constrained, variable capacity links and energy-constrained operation. These limitations are imposed by the shared nature of the wireless channel, the mutual interference between nearby nodes and the resourceconstrained devices. Furthermore, the dynamic topology in this kind of networks causes frequent link failures and high error rates, which makes even more difficult to maintain a desired degree of Quality of Service (QoS). With the massive demand of video content from mobile devices, it has become very necessary for MANETs to have an efficient routing and QoS mechanisms to support the transmission of multimedia content. An optimal strategy to support applications with real-time requirements could be the use of additional control mechanisms to adapt the processes of encoding, transmitting or decoding, depending on network status. However, conventional transport and routing protocols in mobile ad hoc networks do not provide a suitable connectivity service to support higher bandwidth and quality requirements. Most of the standardized routing protocols for MANETs have been designed to find a feasible route from a source to a destination without taking into account the available resources in the network or specific requirements of an application. Therefore, the established routes using these routing protocols cannot interact with the source node to adjust the transmission rate. Consequently, the application must send its data using a fixed sending rate and cannot take advantage of the adaptation feature inherent in some coding techniques such as scalable video coding (H.264/SVC) and multiple description coding. In addition, without knowing the bottleneck throughput, the source may send much more data than the bottleneck node on the route can transmit. The overwhelmed node must drop data, which wastes a considerable amount of energy and needlessly consumes bandwidth.

Video transmission may be improved by means of additional mechanisms and crosslayer techniques in the routing protocols in order to solve or mitigate the limitations of MANETs.

In this context, the main objective of this thesis work is to:

Improve Quality of Service and Quality of Experience in video streaming service over mobile wireless ad hoc networks by means of new routing protocol improvements, which increase routes stability and allow video application adapts its transmission rate according to the network conditions to avoid video degradation.

In order to achieve the main objective, the specific objectives considered are to:

01. Evaluate the video quality degradation regarding objective (QoS) parameters in video streaming services over mobile ad hoc wireless networks. Different scenarios and conditions will be assessed to obtain results.

02. Propose a QoS routing protocol based on adaptive feedback scheme, which improves the reliability of packet transmission and the maintenance of routes. This routing protocol will be based on the well-known reactive protocol AODV, which is 
commonly used in wireless ad hoc networks. In particular, the improvements will be based in two mechanisms: one for the estimation of the available bandwidth in each node and the other for the prediction of the consumed bandwidth for a route of multihop. Resulting routing protocol will be evaluated as regard performance parameters and will be compared with other similar QoS routing protocols.

03. Improve transmission throughput despite node mobility and route breakages, which are two of the main reasons of packet losses in mobile ad hoc wireless networks. This will be carried out by designing a new route recovery mechanism based on efficient algorithm to improve the rate of link failures and the connection setup latency during a video transmission. This algorithm will consist of an adaptive technique that uses a session cache table where routing information is stored to detect link failures in a route and re-establish the connections taking into account the QoS conditions established during the route discovery phase.

04. Propose a dynamic adaptive video streaming service over ad hoc networks with traffic congestion. This algorithm will count on client feedback for adapting video bitrate at source taking into account the available bandwidth. Hence, video streaming server will be able to adapt the encoding bitrate to the network status to avoid packet losses and video interruptions. In order to assert the Quality of Services improvements in video transmissions, coding techniques such as H.264/SVC will be evaluated using the routing protocol proposed through the assessment objective metrics, such as PSNR.

\subsection{Contributions}

The main contributions of this thesis can be summarized as follows:

1. A new comprehensive QoS routing protocol, named AQA-AODV (Adaptive QoSAware for Ad hoc On-demand Distance Vector) have been developed. This protocol provide some QoS mechanisms during the discovery and maintenance of routes as well as a novel adaptive feedback scheme in order to provide information to the application about the network conditions.

2. Implementation of an algorithm that allows nodes along the path from source to destination node to know their available resources (in terms of bandwidth).

3. Development of a route recovery mechanism, which tries to re-establish connection to destination after a link failure, with the QoS conditions that had been negotiated during the initial route discovery phase

4. An adaptation method for exploiting the layered scheme of the scalable video coding (SVC) and the QoS mechanisms of AQA-AODV in order to adjust the bit rate of the video source adding or removing SVC layers from the original video stream based on the estimation of the available bandwidth. The integration of AQA-AODV and SVC provides a suitable system for supporting a networkadaptive strategy where video stream can be adapted avoiding network 
congestion and achieving a significantly improvement in the quality of the transmitted video.

5. Development of a new simulation framework, called SVCEval-RA, which supports simulation experiments that involve rate-adaptive video transmission of using the H.264/SVC standard.

6. Implementation of a gateway discovery algorithm to improve the interoperability of MANETs and infrastructure-based networks. With the proposed discovery mechanism, AQA-AODV is able not only to establish routes according to the QoS requirements of the requesting node in a MANET, but also is able to establish communications with a host in a wired network through gateways. The proposed algorithm incorporates the available bandwidth as a metric during the gateway selection and a dynamic adaptation of some operational parameters such as the size of the proactive area and the frequency of the advertisement messages.

Moreover, this thesis has led to the publication of scientific journal papers, conference presentations and book chapters. A detailed list of publications derived from this work is presented in Appendix A.

\subsection{Layout of this thesis}

This thesis is organized into the following chapters:

- $\quad$ Chapter 1 - Introduction

In this chapter an overview of some basic concepts of wireless ad hoc networks is given. In addition, it is provided a background information about their applications, features and open issues, which motivated this work. This conceptual framework provides the basis for defining the research problem and establishing the objectives of the thesis.

- $\quad$ Chapter 2 - Video streaming over MANETs

This chapter discusses an experimental study the key issues and limitations for the provision of video streaming services over wireless ad hoc network. In particular, the problem of the route capacity is quantitative characterized. The analysis performed in this chapter lays the basis to understand the reasons of the quality degradation incurred during the video delivery over wireless ad hoc networks. The Chapter 2 is aligned with objective $\mathrm{O} 1$.

- $\quad$ Chapter 3 - QoS routing protocols in MANETs

This chapter proposes a new routing protocol named AQA-AODV, which creates routes according to QoS requirements of the application layer. Components of the architecture of AQA-AODV are described in this chapter as well as the results obtained from the performance evaluation of the proposed protocol. Chapter 3 is aligned with objectives $\mathrm{O} 2$ and $\mathrm{O} 3$. 
- $\quad$ Chapter 4 - Adaptive Video Streaming over MANETs using AQA-AODV

This chapter analyses the use of AQA-AODV in the provision of adaptive video streaming over MANETs in combination with the Scalable Video Coding (H.264/SVC). In addition, an evaluation framework for adaptive scalable video streaming is also presented. Chapter 4 is aligned with objective $\mathrm{O} 4$.

- Chapter 5 - Gateway discovery extension for AQA-AODV

In this chapter, it is described a gateway discovery algorithm implemented as an extension of the AQA-AODV routing protocol in order to improve the interconnectivity between MANETs and the infrastructure-based networks, such as Internet and cellular networks. 



\section{Chapter 2 \\ Introduction to Video Streaming over MANETs}

Video transmission over wireless ad hoc network presents many issues as a result of the particular characteristics of MANETs, such as dynamic network topology, variable link capacity, nodes mobility, energy constraints and lack of centralized infrastructure. These features makes the QoS provision over these networks a really challenging target.

This chapter analyses the key issues and limitations for the provision of video streaming services over wireless ad hoc network. In particular, the problem of the route capacity is studied through simulation experiments, which allows us to understand the reasons of the quality degradation incurred during the video delivery over wireless ad hoc networks. In addition, a briefly revision of the video streaming techniques is carried out, summarizing their advantages and technical requirements.

\subsection{Achievable transmission rate in wireless ad hoc networks}

One of the limitations of wireless ad hoc networks is the achievable capacity due to the fact that nodes cannot simultaneously access the shared medium. More specifically, when a node is transmitting a packet, neighbour nodes within its Interference Range (IR), have to keep silent. This fact degrades the wireless data rate. Even more, when a transmission is established, the nodes must cooperate to forward the packets through the network, which means that the available throughput on each host is limited not only by the access channel, but also by the forwarding load. In addition, the RTS/CTS mechanism that avoid packet collisions adds a significant amount of protocol overhead that also results in a decrease in network throughput. As a result, an overall degradation in data rate is produced. This effect has been already studied by Bianchi [30] and Gupta and Kumar [31].

An additional issue is presented when the hop count of a route increases since the maximum throughput of one flow decreases substantially and falls down, not only 
because of the overhead of MAC layer, but also because of the mutual interference between packets of the same flow. This effect is called Intra-flow Contention [32] and represents the contention between packets from the same flow being forwarded at different hops along a multi-hop path, causing the actual bandwidth consumption. Figure 2.1 illustrates this issue in a chain of static nodes. Note that interference range is usually higher than transmission range. Therefore, it may interfere with further nodes. In this example, transmission from node 4 to node 5 interferes in other transmissions along the node chain. On the one hand, it will block any other transmission originated at nodes 2, 3, 4, 5 and 6 . On the other hand, it might corrupt data transmission from node 1 to 2 , since it interferes in the reception at node 2 .

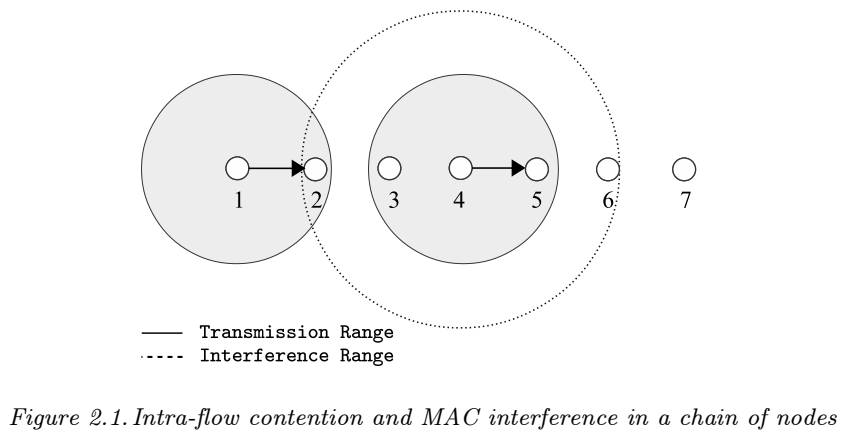

Usually, multiple-hop flows have to contend for the medium at each hop on its way to the destination node. Consequently, flows that extend over more hops will spend more time on competing with more nodes in the network. Then, packets forwarded on a longer path are more likely to be dropped than those on a shorter path. In order to show the effect of the path length caused by the intra-flow contention, a chain scenario with different chain lengths has been analysed. The network topology and the simulation parameters are described in Figure 2.2. Simulated scenarios are comprised of stationary nodes located a certain distance away one another enough to have only next hop in range.

Simulations have been performed in order to assess the aforementioned decrease of throughput along a single linear network topology of nodes where the source of traffic is the first node, destination is the last node and the packets are being forwarded through the intermediate nodes. The source node sends data as fast as its MAC allows it. In this scenario, only adjacent nodes are in transmission range of each other. The simulation results were obtained with a confidence level of $95 \%$. Figure 2.3 depicts this decrease of the overall throughput depending on the total amount of nodes that are taking part in the path to destination. Note that although there is only a single transmission, as the chain length grows the maximum throughput achieved at the receiving node decreases, as stated before, due to the contention of the packets of the same flow trying to access the shared medium. The fact of transmitting at the maximum channel rate may cause a situation of maximum intra-flow contention and, therefore, it could not be the optimal transmission rate. 


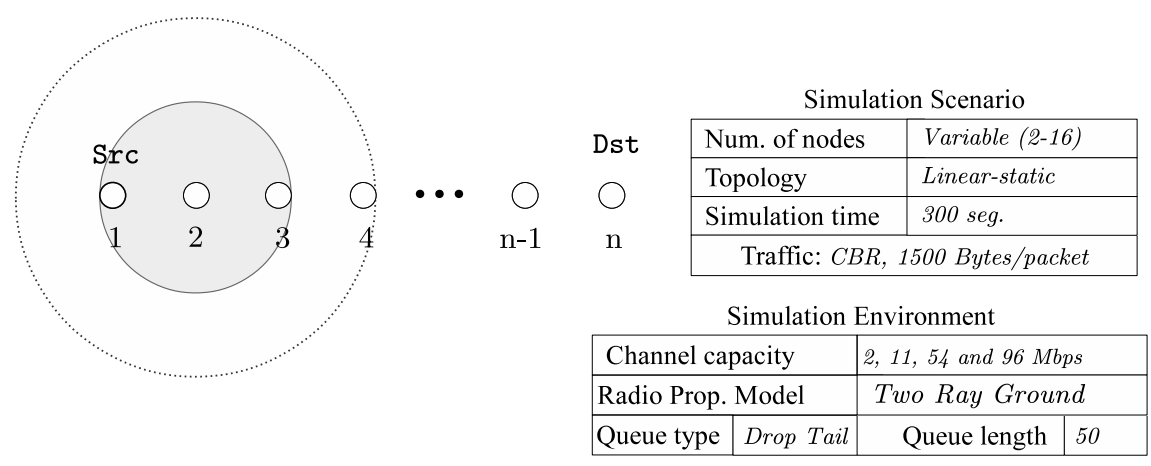

Figure 2.2. Main simulation parameters and network scenario

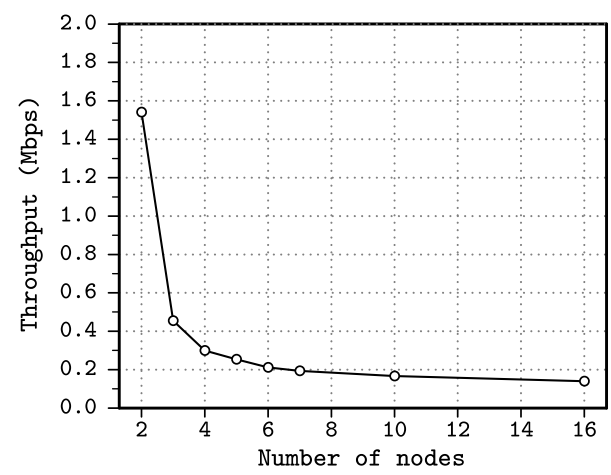

(a)

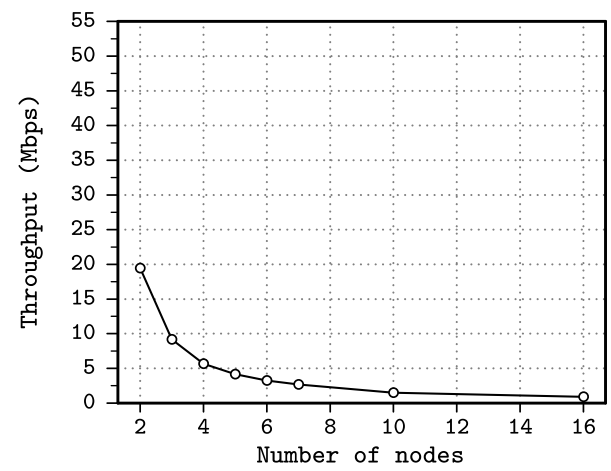

(c)

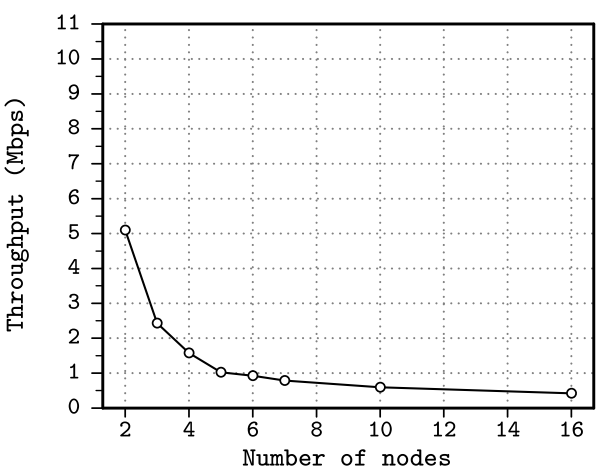

(b)

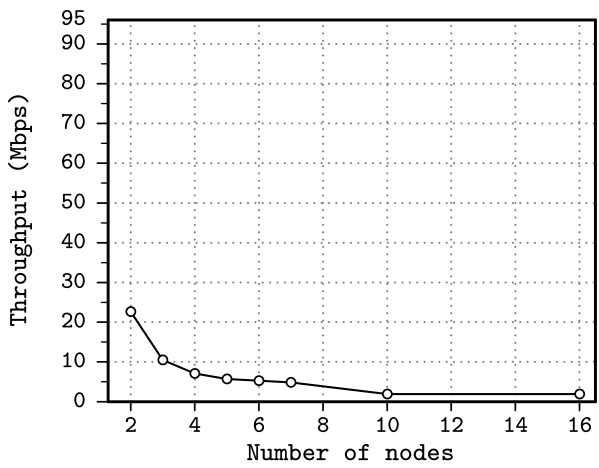

(d)

Figure 2.3. Average throughput achieved in the chain, as a function of the chain length in wireless ad hoc networks for 802.11b@2Mbps (a), 802.11b@11Mbps (b), 802.11g@54Mbps (c) and 802.11n@96Mbps (d)

Moreover, by comparing relative values from the Figure 2.3a, Figure 2.3b, Figure 2.3c and Figure 2.3d it can be noted that there are significant differences in the chain throughput achieved for the different channel capacities mainly due to the radio link 
and the particularities of the different modulation schemes. For instance, in 802.11b@2Mbps when the chain has only two nodes, the maximum throughput is 1.53 Mbps of effective network capacity, instead of the maximum capacity ( $2 \mathrm{Mbps}$ ), which is only the physical layer data rate. In fact, effective throughput will never be as much as this upper limit due to several factors, such as the overhead of headers, ACK packets, additional inter-frame timings and, if it would be the case, RTS/CTS packets. Even in the best case, throughput would never overtake the Theoretical Maximum Throughput (TMT) [33], [34]. Hence, this throughput represents the 77\% compared with the standard maximum capacity (2 Mbps). In the case of 802.11b@11Mbps, 802.11g@54Mbps and 802.11n@96Mbps, the throughput achieved when the chain has only two nodes is 5.09 Mbps, 19.4 Mbps and 22.6 Mbps respectively, which corresponds to about 46\%, 35\% and $23.5 \%$, respectively.

These results are not unexpected at all, but they have to be taken into account when analysing wireless networks and specifically when assessing services that consume an important amount of bandwidth, such video streaming services. Medium contention and transmission interferences do worsen the average network throughput when several data sources are competing for the medium access, but this contention exists even when only a single transmission is carried out through the network (or it is locally isolated in the network).

As explained above, there is a mutual interference that is unequally experienced by nodes, which lead to the delivery packets by node 1 with a data rate greater than the forwarding rate of the subsequent nodes. This fact causes a significant increment of the lost packets (see Figure 2.4).

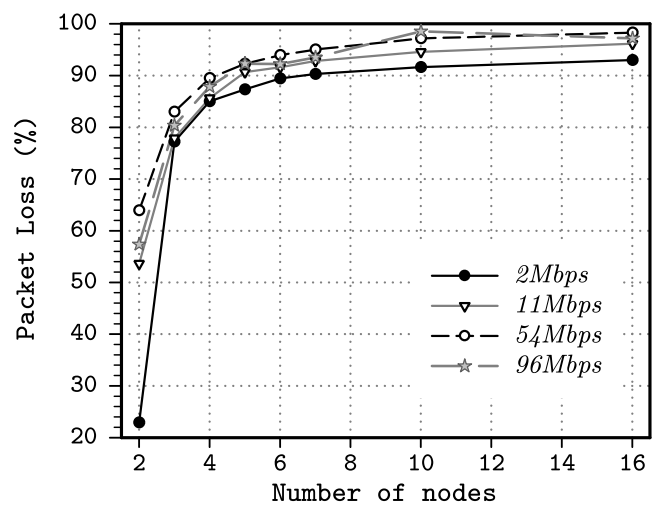

Figure 2.4. Percentage of lost packet as a function of number of nodes

In order to evaluate the channel response for different transmission rates, a scenario consisting of a chain of 7 nodes has been simulated varying the source data rate. Figure 2.5 shows the network response while increasing the offered load. The average throughput achieved varies practically linear until a certain value of offered load. A maximum value for the throughput curve is obtained at this data rate $(0.4 \mathrm{Mbps}$ in 802.11b@2Mbps, 1.2 Mbps in 802.11b@11Mbps, 5.1 Mbps in 802.11g@54Mbps and 9.1 
Mbps in 802.11n@96Mbps). From this offered load, throughput decreases and is maintained steady.

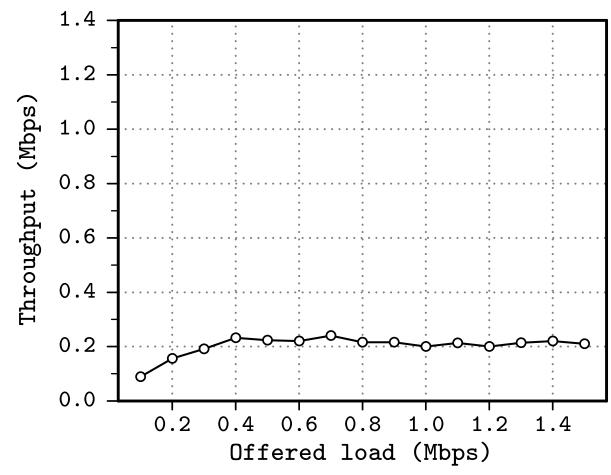

(a)

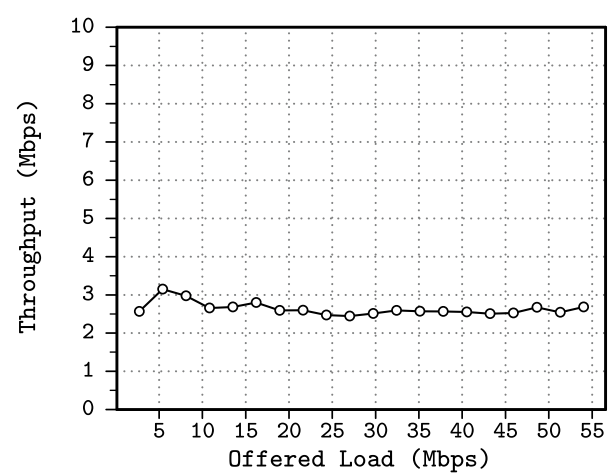

(c)

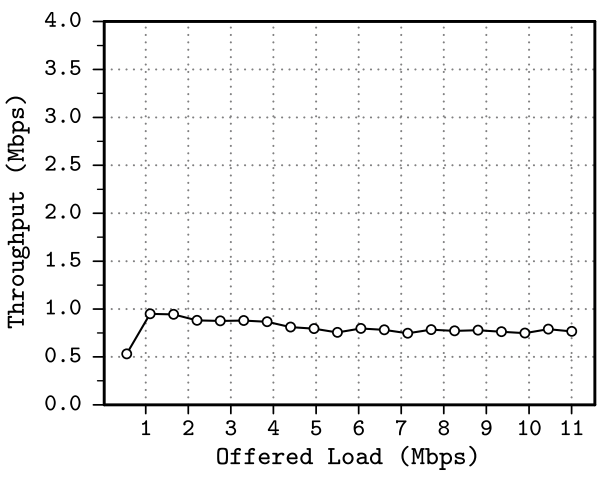

(b)

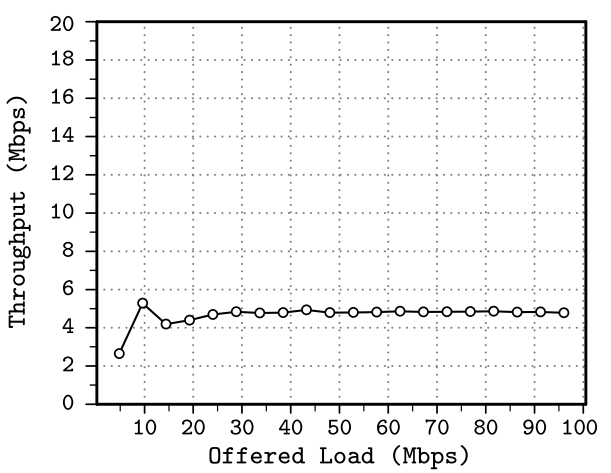

(d)

Figure 2.5. Average throughput vs. offered load in a 7-node chain network for 802.11b@2Mbps (a), 802.11b@11Mbps (b),802.11g@54Mbps (c),802.11n@96Mbps (d)

Differences between 802.11 modes in terms of packet losses can be seen in Figure 2.6. In this figure, the points of maximum throughput are marked with a circle for each curve. In 802.11@54Mbps, the throughput corresponds linearly to the data rate used until the drop. Percentage-wise, the other modes reach their maximum throughput value with more offered load but the fall is not so abrupt. However, it can be observed that the offered load is not always achieved even before the upper bound rate. Specifically, the percentage of lost packets is $0 \%$ when offered load is 5\% in $802.11 \mathrm{~g} @ 54 \mathrm{Mbps}$ and 802.11n@96Mbps, 9.2\% for 10\% of offered load in $802.11 \mathrm{~b} @ 11 \mathrm{Mbps}$, and $41 \%$ for $20 \%$ of offered load in $802.11 \mathrm{~b} @ 2 \mathrm{Mbps}$. This has to be seen as an upper bound rate characteristic of wireless ad hoc networks, which are not able to transmit at a higher rate due to physical limitations. The high packet loss rate can be explained as follows. Considering again the example of Figure 2.1, in which the mutual interferences between nodes in a chain of 7 nodes are illustrated, it can be observed that node 3 experiences interference from five other nodes, while node 1 is interfered by only three other nodes. This fact allows node 1 to inject more packets into the chain than the subsequent nodes 
can forward, as detailed in Figure 2.7. These packets are eventually dropped at nodes 2 and 3. Therefore, overall packet losses in this scenario increase as the sending rate of the node 1 increases.

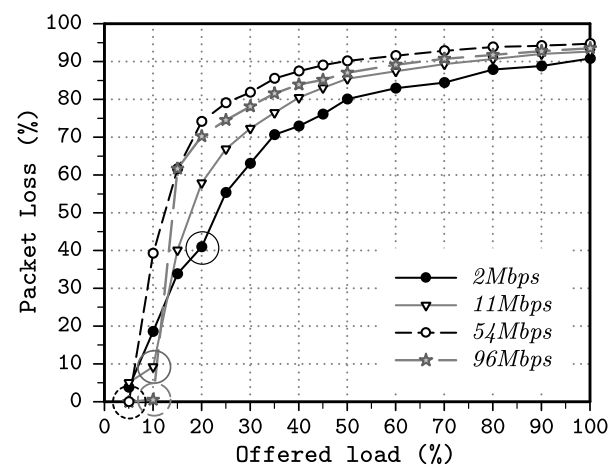

Figure 2.6. Percentage of Lost Packets for 802.11 mode

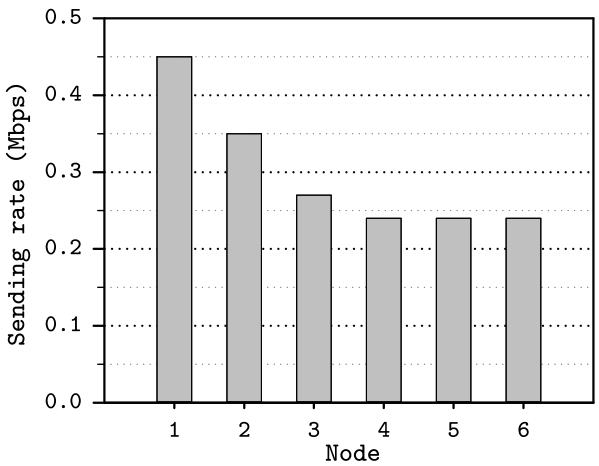

Figure 2.7. Sending rate per node in a chain of 7 nodes

The preliminary studies presented in this chapter confirm that wireless ad hoc networks may present a continuous fluctuation of the end-to-end throughput as well as a significant increasing of network congestion (high values for packet delay and packet loss), which represents a challenging scenario for video transmission. In addition to constraining factors as MAC overhead, node interference and intra-flow contention, the mobility of nodes may causes additional packet losses and, therefore, also may degrade delivered video quality. All these issues have been considered in the formulation of the proposals that this thesis presents.

\subsection{Adaptive video streaming}

There are two main methods for video delivery: traditional video streaming (that includes simple progressive download and real time streaming techniques) and adaptive streaming. In the traditional techniques, video is sent as a flow of packets using a protocol that is in charge of establishes a session between the video provider and client. This technique is known as a real time streaming. Also, the progressive download 
technique is considered as traditional video transmission technique. In this technique users request video content which is downloaded progressively into a local buffer. As soon as there are sufficient data the media starts to play. If the playback rate exceeds the download rate, then playback is delayed until more data is downloaded.

However, these traditional video transmission schemes lack of the necessary flexibility and adaptability since the video file is delivered to the end-user with a constant bit rate through a persistent source-destination connection. Thus, if a particular transmission channel experiences congestion, the quality of the video can significantly degraded (see Figure 2.8)

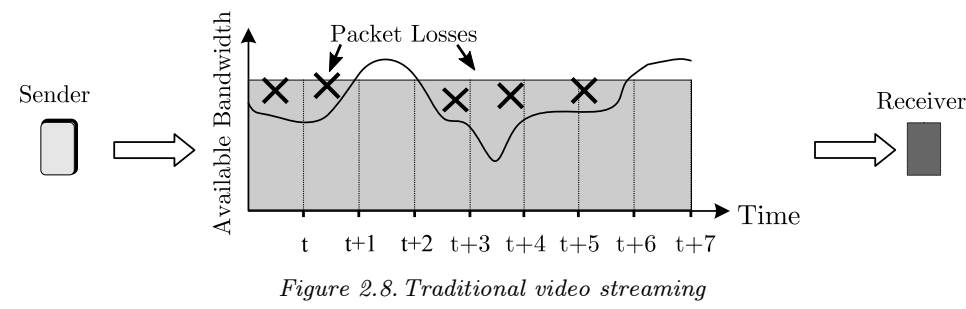

Adaptive streaming [35]-[37] is a technique which estimate the available bandwidth and CPU capacity in order to adapt the video quality that is provided to the user. To implement the idea of adaptive transmission, three types of techniques have been proposed. One possibility is the transcoding, which consist on transcode from one encoding to another to match a specific bit rate. However, there are some important disadvantages as the high cost of transcoding due to the fact that the raw video content requires to be adapted several times for different quality requests. Consequently, scalability decreases since transcoding needs to be performed for every different client. Therefore, because of lack of flexibility and the high computational requirements of a real-time transcoding system, the transcoding technique is not a suitable solution for mobile ad hoc networks [38].

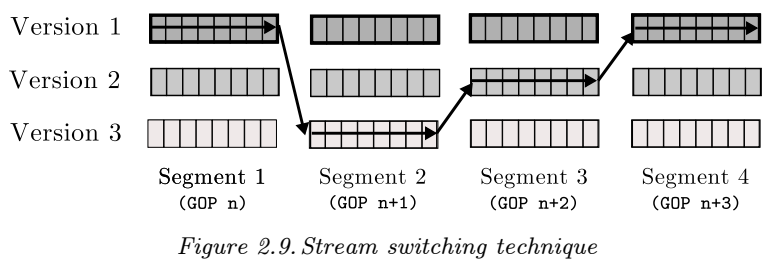

The second type of adaptive video transmission technique is based on the idea of stream switching. This technique encodes the raw video content at several different increasing bit rates, generating $j$ versions of the same content. Figure 2.9 illustrates an example of this technique, where 3 versions of a same video are generated. Each video version is also divided into segments of the same duration (e.g. using the segmentation based on GOP). An algorithm must dynamically choose the video version that matches the available bandwidth of the route. Although this method minimizes processing costs, storage and transmission requirements must be considered because the same video 
content is encoded $j$ times. Other disadvantage of this method is the coarse granularity since there is only a discrete set of levels.

The third type of adaptive video transmission technique employs Scalable Video Coding (H.264/SVC) [39], which has attracted great interest by researchers to improve video streaming over wireless networks [40]-[42]. The scalable video coding (H.264/SVC) provides features to generate different representations of the same video integrated within a same bit stream. A video encoded using the $S V C$ standard has a layered structure where the layers correspond to different quality, spatial or temporal representations. A SVC video is composed of a base layer, which corresponds to the lowest representation, and one or more enhancement layers that increase the video quality when these are added to the base layer. The layered scheme of SVC can provide higher robustness during video streaming over networks with high packet loss rates. This robustness allows the receiver to decode the video even if there is a high packet loss rate (see Figure 2.10a) because if enhancement layers are lost, the receiver may still decode received video as long as the base layer is correctly received. However, it is not a bandwidth-efficient solution because of the large amount of data sent and lost due to packet losses. Therefore, in order to adaptively control the bit rate of the video source, the adoption of cross-layer mechanisms in video streaming is required. Cross-layer solutions involves information exchange and optimization between the application layer operations (e.g. source coding), the network layer and the transport protocols in order to obtain optimal combinations of video bit rates and routing policies. These solutions can be used for supporting adaptive scalable video streaming schemes. In fact, the combined usage of $S V C$ and adaptive streaming techniques can significantly contribute to increasing the quality of video streaming services while the bandwidth efficiency is achieved (see Figure 2.10b).

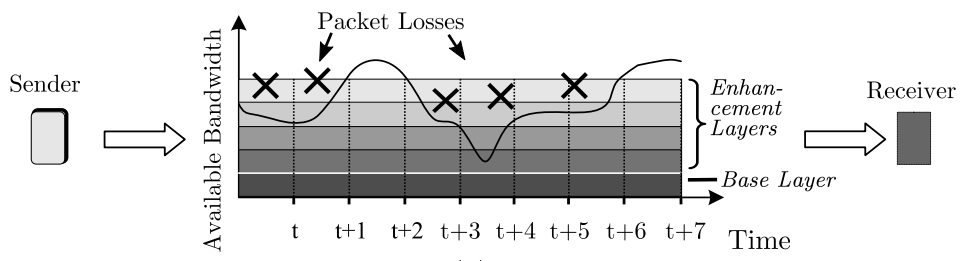

(a)

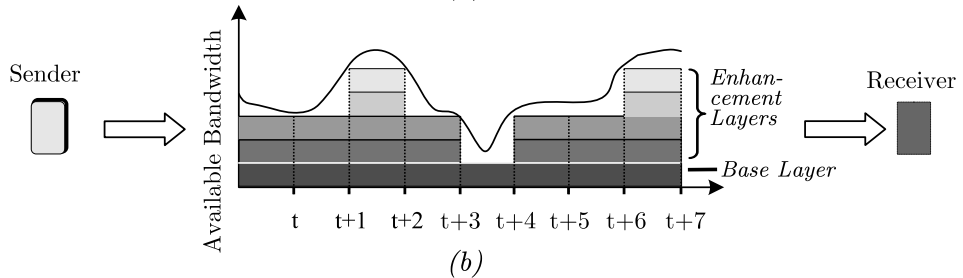

(b)

Figure 2.10. Comparison of the scalable video streaming (a) and the adaptive scalable video streaming (b)

This thesis focuses in provide a realistic solution following the scheme illustrated in Figure 2.10b. Therefore, the next chapters will introduce the developed elements required for implementing such solution. Finally, in Chapter 4 a cross-layer solution for 
supporting adaptive video transmissions using the scalable video streaming will be presented.

\subsection{Video streaming over MANETS}

The development of video streaming services on wireless ad hoc networks is a challenge task as a consequence of different limitations such as bandwidth-constrained, variable capacity links and energy-constrained operation. Moreover, the dynamic topology of nodes causes frequent link failures and high error rates. Figure 2.11 summarizes the issues that affect the video quality when a video stream if delivered through a MANET. On the other hand, because of their nature, video streaming services are very sensitive to packet loss and delay, which further increases the difficulty to support real-time video on wireless ad hoc networks. In order to quantify the complexity incurred in the provision of video streaming services on wireless ad hoc networks, a diagnostic study is presented. Firstly, the linear network formed by a chain of nodes has been simulated. The simulation scenario is similar to that depicted in Figure 2.2. Wireless network uses the IEEE802.11 protocol in the MAC layer working in the distributed coordination function (DCF) mode with a channel data rate of $2 \mathrm{Mbps}$.

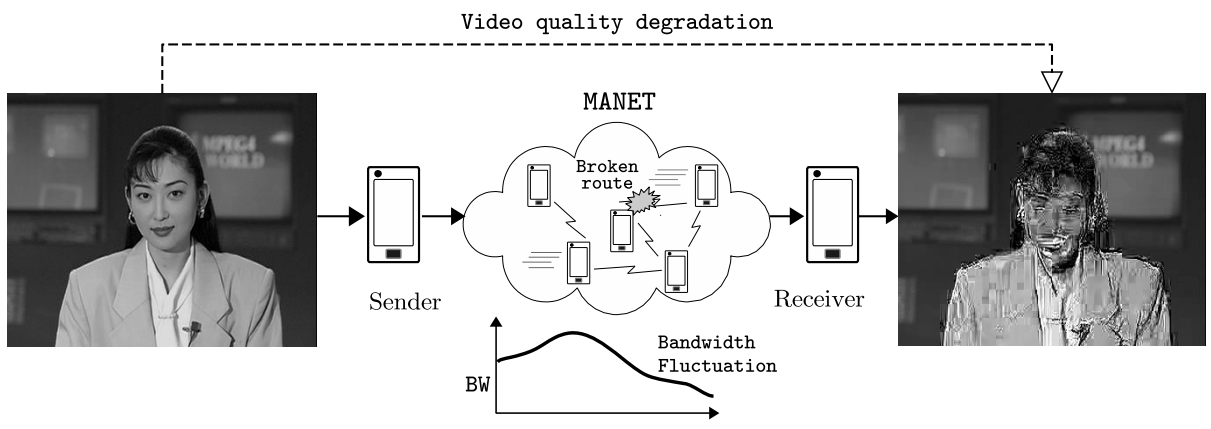

Figure 2.11. Video quality degradation in multi-hop wireless networks

The first node of the chain is the video source, which transmits a video of $60 \mathrm{~s}$ of duration, 352x288 pixels (CIF), with a frame rate of $15 \mathrm{fps}$. The video has been created by concatenating the test sequences akiyo, news and foreman [43], available on the Internet and commonly used for video assessment. This video was encoded at different average bitrates (100 Kbps, $300 \mathrm{Kbps}$ and $500 \mathrm{Kbps}$ ) with a tolerance of $5 \%$. It has been encoded using H.264/AVC with a GoP size of 12 frames, without B-frames (bidirectional).

Since it is not advisable to use video file directly in the simulations as a traffic source, mainly due to the great amount of data to be processed, the video trace technique have been used. This method extracts information from the video and then such information is saved in a text file. With this information traffic is generated as it were real video. Video traces contains useful information, such as packet size, number id and timestamp about the packets to be transmitted. Video sources are simulated from these traces and then, new traces are obtained from the received video. This process is carried out using Evalvid tools [44], [45], which is a well-accepted platform for evaluation of the quality of 
video transmitted over a real or simulated communication network. Received video is reconstructed from the original sequence taking into account the source and the received traces. Logically, at this point, lost packets, which do not appear in the received trace, cannot be used to reconstruct the video. Finally, both original and reconstructed videos are available for a full-reference analysis, for instance, calculating the PSNR (Peak Signal-to-Noise Ratio). In addition, other performance metrics also can be calculated, as the average packet delay, throughput and packet loss rate. These results are illustrated in Figure 2.12 for the different bitrates used.

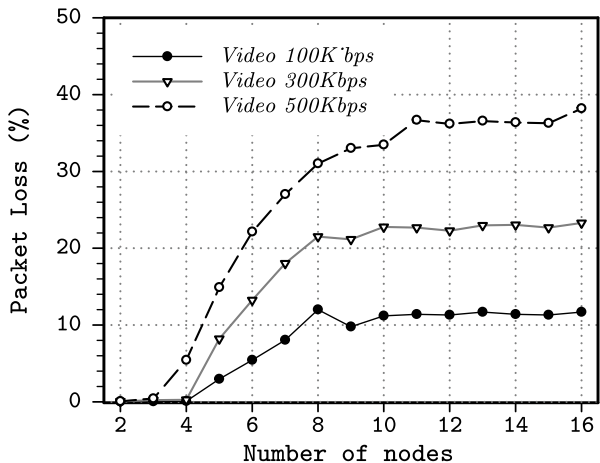

(a)

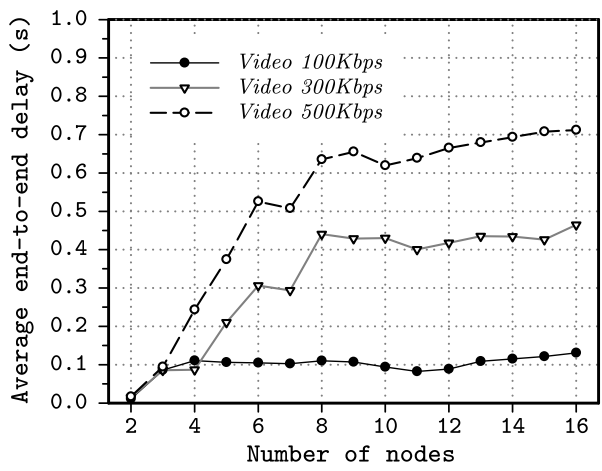

(c)

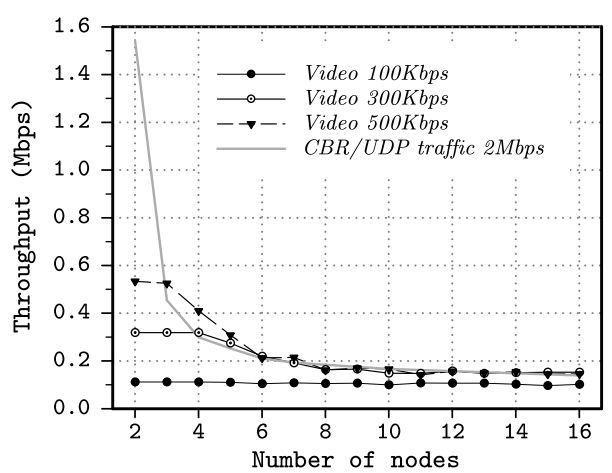

(b)

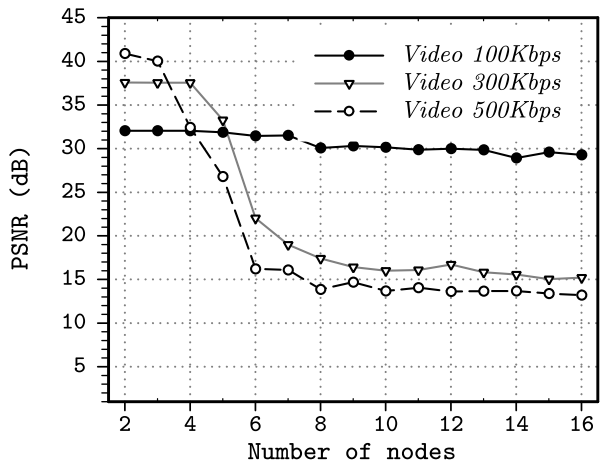

(d)

Figure 2.12. Packet Losses (a), chain throughput (b), packet delay (c) and PSNR (d), as a function of the chain length

In general terms, there is a clear trend in the degradation of the link quality as the number of nodes in the chain increases. Concerning packet losses, Figure 2.12a shows that a video with a higher bitrate starts to lose packets before than another with lower bit rate when number of hops increases. For instance, when the video version of 100 Kbps is transmitted, the packet loss incurred is about $14 \%$ in an 8-node chain, $21.5 \%$ for the version of $300 \mathrm{Kbps}$ and $31 \%$ for $500 \mathrm{Kbps}$. The curves in Figure 2.12b show the throughput achieved in video transmission as well as the maximum throughput achieved for a CBR UDP transmission of $2 \mathrm{Mbps}$ (recall that this is the maximum of the standard used) in order to compare with video results. 
By analysing the throughput achieved, it is observed that the video stream of $100 \mathrm{Kbps}$ is forward in all simulated scenarios incurring in low packet losses. On the contrary, the video stream of $300 \mathrm{Kbps}$ cannot efficiently transmitted for routes with 3 or more nodes of length and the video of $500 \mathrm{Kbps}$ in chains with 4 or more nodes. This fact lead to network congestion such as is demonstrated by the growing values of lost packets and average delay (see Figure 2.12b and Figure 2.12c). For example, during the transmission of the video version of $500 \mathrm{Kbps}$ the packet delay obtained reached from $380 \mathrm{~ms}$ until $700 \mathrm{~ms}$ in chains with more of 5 nodes. These values of packet delay can be unaffordable in some kinds of real-time video transmissions. Although it seems that throughput curves for the video transmissions of $300 \mathrm{Kbps}$ and $500 \mathrm{Kbps}$ get adjusted to the maximum throughput reachable (i.e. achieved throughput with UDP traffic) in reality, what happens is that a high percentage of packets is dropped due to network congestion. Minimal differences can be appreciated between UDP and video traffic, which can be mainly due to the difference of packet sizes and therefore, differences in back-off times to access the medium. Hence, it would be logical to try to find a solution that could reduce the number of dropped packets and the packet delay adapting the sending rate to the maximum traffic that can be efficiently supported by the route to the destination, without causing more interferences or contention, as addressed in Chapter 3.

Figure 2.12d shows the PSNR of the received sequences. As long as PSNR takes into account both encoding and transmission losses, the higher the bitrate, the better the PSNR, when transmission losses are negligible or zero. However, when packet losses occur, videos with higher bitrate are more affected because the percentage of received packets is decreased dramatically. As observed in Figure 2.12d, the video stream with bit rate below the maximum throughput achievable obtained the best video quality, despite it stream was transmitted a low bit rate that the other video flows. This fact suggests that if an effective adaptation of the data rate to the network condition is performed, quality of a video transmission could be significantly improve.

\subsubsection{Conclusions}

The results obtained in these simulations suggest that video delivery over MANETs must be designed under the paradigm of content adaptation in order to adjust the traffic flow to the available resources. This idea requires the development of a cross-layer strategy that provides information exchange and optimization between the routing layer, the transport protocol and the application layer operations.

In this sense, this thesis presents novel proposals that improve the network conditions to video streaming addressing some of the most important open issues in mobile ad hoc networks. The details of the first proposed solution will be explained in next Chapter, which is focused on providing QoS mechanisms to the routing layer.

\subsection{Summary}

In this Chapter, a revision of the main issues and challenges related to the provision of video streaming over mobile ad hoc networks was presented. Specifically, the effects of the mutual interference between nodes, the MAC overhead and the contention scheme 
of the 802.11 standard over the degradation of the maximum throughput achievable along a route. In addition, the channel response for different transmission rates was also analysed. After analysing the Due to this fact, a network-adaptive video streaming technique based on the scalable video coding was also explored in this Chapter.

A set of simulation experiments were performed in order to characterize the real impact of the multi-hop communications over the data transmission performance. Similarly, a preliminary assessment of video streaming over wireless ad hoc networks were carried out. The obtained results demonstrate that MANETs have a continuous fluctuation of the end-to-end throughput as well as a high packet loss rate. This fact causes a significant degradation in the transmitted videos; thus, an adaptive solution that helps avoid or reduce the network congestion adjusting the sending rate would be addressed. 


\section{Chapter 3}

\section{AQA-AODV: A cross-layer routing Protocol for MANETs}

One of the major challenges for the transmission of time-sensitive data over MANETs is the deployment of an end-to-end QoS support mechanism. A realistic solution for QoS provision should not separate the routing from QoS management since it can involve the selection of inefficient routes and, thus, reduce the likelihood of meeting the QoS requirements of the established communications in the ad hoc network. This chapter proposes a new QoS routing protocol (named AQA-AODV), which creates routes according to QoS requirements of the application layer. This proposal introduces link and path available bandwidth estimation mechanisms and an adaptive scheme that can provide feedback to the source node about the current network state, to allow the application to appropriately adjust the transmission rate. In the same way, an enhancement of route recovery process is integrated into the AQA-AODV protocol, which provides a mechanism to detect the link failures in a route and re-establish the connections taking into account the conditions of QoS that have been established during the initial route discovery.

\subsection{An overview on routing protocols in mobile ad hoc networks:}

The conventional routing protocols for mobile ad hoc networks have been designed to find a path, between two communicating nodes, without taking into account its quality. These protocols discover and maintain paths that are generally established according to the minimum hop-count metric. Some protocols of this type, such as AODV (Ad hoc OnDemand Distance Vector Protocol) [46], OLSR (Optimized Link State Routing Protocol) [47] and DSR (Dynamic Source Routing Protocol) [48] were selected as standards by the IETF (Internet Engineering Task Force) organization. However, these protocols are now the starting point for new routing protocols where providing QoS is a key objective. In this subsection, a brief introduction to the traditional routing protocols for mobile ad hoc networks are presented and later some QoS routing protocols will be introduced. 
The routing protocols for mobile ad hoc networks can be can be classified according to different criteria. The most common classification is based on the routing information update mechanism. Hence, routing protocols can be driven either by a routing table (proactive) or on demand (reactive). Proactive protocols always maintain up-to-date information of routes from each node to every other node in the network. Routing information is stored in the routing table of each node and route updates are propagated throughout the network to keep the routing information as recent as possible. As a consequence, there is a constant overhead due to routing traffic but there is no initial delay in data communications. Some examples of proactive routing protocols are Destination-Sequenced Distance Vector (DSDV) [49] and Optimized Link State Routing (OLSR) [47]. On the other hand, with reactive protocols the route is created only when the source requests a route to a destination or when a broken link is detected. This kind of routing protocol does not need extra control packets for maintenance, although high latency time can be generated when establishing the new route. The most important reactive routing protocols are Ad hoc On-demand Distance Vector (AODV) [46], Dynamic Source Routing (DSR) [48], Dynamic MANET On-demand (DYMO), also known as AODVv2, which is still under standardization process (draft) [50]. Additionally, there are hybrid proposals that combine the elements of proactive and reactive protocols, such as the Zone Routing Protocol (ZRP) [51], and Hybrid ant colony optimization (HOPNET) [52].

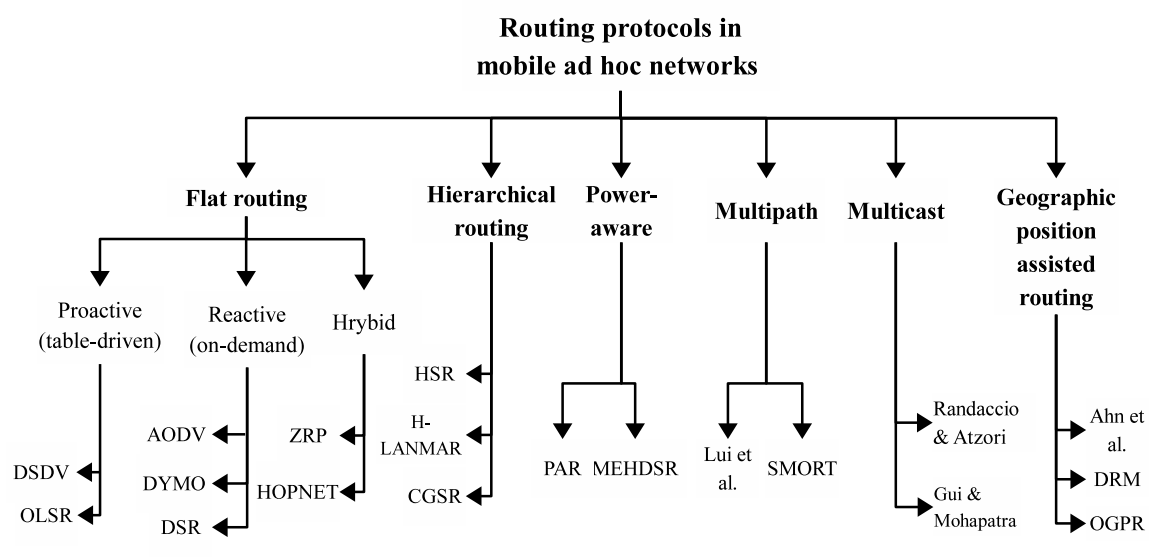

DSDV (Destination Sequence Distance Vector). OLSR (Optimized Link State Protocol), AODV (Ad-hoc On-demand Distance Vector), DYMO (Dynamic MANET On-demand), DSR (Dinamic Source Routing), ZRP (Zone Routing Protocol), HOPNET (Hybrid ant colony optimization), HSR (Hierarchical State Routing), H-LANMAR (Hierarchical landmark routing), CGSR (Clusterhead-Gateway Switch Routing), PAR (Power Aware Routing), MEHDSR (Minimum energy hierarchical dynamic source routing), SMORT (Scalable multipath on-demand routing), DRM (Dynamic route maintenance), OGPR (On-demand geographic path routing)

Figure 3.1. Routing protocols for mobile ad hoc networks: approaches and classification

The Figure 3.1 shown a graphical representation of the classification of the routing protocols for mobile ad hoc networks. Routing protocols can be also classified in flat or hierarchical protocols depending on the way nodes are organized throughout the network. Flat routing protocols establish a flat topology since they assume that wireless 
network is formed by homogeneous nodes, i.e. devices with similar capabilities. On the other hand, hierarchical routing protocols build a hierarchy of nodes, typically through clustering techniques. Nodes at the higher levels of the hierarchy provide special services, improving the scalability and the efficiency of routing such in Hierarchical OLSR [53], Hierarchical landmark routing (H-LANMAR) [54] and Cluster-head Gateway Switch Routing (CGSR) [55].

Recently, another type of routing protocols have been proposed. These protocols prioritize energy consumption and remaining battery life in order to take routing decisions. Some examples of power-aware routing protocols are Minimum energy hierarchical dynamic source routing (MEHDSR) [56] and Power Aware Routing (PAR) [57].

Finally, some proposals aim at improving the performance of routing by means of using geographical information [58]-[60], establishing multicast communication [61], [62] or create multiple routes from source to destination [63], [64].

\subsection{QoS routing protocols for mobile ad hoc networks}

The essential problem in order to support QoS in mobile ad hoc networks, is to find a route with sufficient available resources to meet the QoS constraints and possibly to incorporate optimizations, such as finding the lowest cost or most stable of the routes that meet the QoS constraints. According to these goals, Han et al. [65] define some basic design considerations for a QoS-aware routing protocol. These considerations can be summarized as follows:

1. Resource estimation: is necessary to know information about available bandwidth for performing call admission and QoS adaptation.

2. Resource reservation: an open issue is how to allocate the bandwidth resources and what type of resource reservation may be performed.

3. Route recovery: frequent link failures due to mobility of nodes; therefore, incorporating a fast route recovery scheme into QoS-aware routing is other design consideration.

4. Route selection: QoS-aware routing protocol should consider the information about available bandwidth (end other metrics as delay) when selecting a suitable route.

Several routing protocols have been developed to provide QoS in mobile ad hoc networks taking into consideration one or more of the criteria mentioned above. Most of the QoS routing protocols are the extensions of existing best-effort routing protocols; therefore, they can be also classified into two different categories: on-demand (reactive) and table-driven (proactive). Numerous reactive and proactive QoS routing protocols have been proposed for MANETs recently. Nevertheless, in this thesis, focus is on reactive QoS routing protocols and mainly those solutions based on the well-known AODV routing protocol. For example, Zhen and Wenzhong in [66] have proposed an approach which uses AODV as routing protocol within a TDMA (Time Division Multiple 
Access) network. However, TDMA has a less efficient controlled access scheme because of the lack of infrastructure and the peer-to-peer nature of ad hoc networks.

Other QoS routing protocols are based on the IETF draft [67] (called QAODV) which describes the format and extensions to provide QoS support in AODV. Some approaches of this kind are described in references [4], [5], [68], [69], [6]. They are based on the model of admission control of QAODV without any mechanism of feedback. Therefore, the source cannot know the available resources of the network. Moreover, the initial QoS conditions are not maintained after link failures due to the lack of a suitable route recovery algorithm. Another solution based on AODV is the BEQR protocol (Bandwidth Estimation QoS Routing protocol) [70], which uses two kinds of schemes, a feedback scheme and an admission scheme. Additionally, Kumar et al. in [71] proposed a solution called EQOS-AODV to enhance AODV. They extended the Request (RREQ) and Reply $(R R E P)$ packets and the routing table to store information about the available resources. In addition, they proposed a new table called "neighbour table", used to store and update the routing table periodically by interchange of HELLO packets containing QoS information. Therefore, each node has to maintain two tables: neighbour table and routing table. This approach does not reduce significantly the packet delay; on the contrary, it increases the processing load of the nodes due to the fact that these have to update two tables. Finally, others QoS routing protocols for MANETs based on AODV are described and compared by Gulati and Kumar in [2] and Moussaoui et al. in [72]. Nevertheless, these approaches do not provide suitable mechanisms that allow the source application to tune some parameters in order to optimize the transmission of multimedia flows.

Some QoS routing protocols for wireless ad hoc networks are summarized in Table 3.1. Each protocol is referred by its name and a reference to the paper where it was defined. Only reactive protocols have been considered which are listed in alphabetical order according to their names. The main features described for each protocol are: the QoS metric used during the route discovery process, the pre-requisites or assumptions involved in the use of each protocol and a brief description of their operation.

\subsection{AODV and QAODV routing protocols}

AODV and QAODV are important references to contrast the performance of the proposed routing protocol. Therefore, their main characteristics are summarized as follows. The AODV (Ad hoc On-demand Distance Vector) protocol [46] is a widely accepted routing protocol for MANETs which uses a broadcast route discovery mechanism. When a source needs a route to a destination, it broadcasts a Route Request ( $R R E Q$ ) packet in search of a route. A node after receiving an RREQ sends a Route Reply $(R R E P)$ packet to the source, if it either is the destination node, or has an active (fresh) route to the destination. Otherwise, it rebroadcasts the $R R E Q$ packet to its neighbours and creates a reverse route entry for the source. An intermediate node receiving $R R E P$ packet creates a forward route entry for the destination and further forwards the RREP packet toward the source using its reverse route entry. Finally, when source receives 
multiple RREPs, it selects the RREP with the highest destination sequence number. Sequence numbers are used to ensure fresh and loop-free routes.

Table 3.1. QoS routing solutions - Capability and prerequisites

\begin{tabular}{|c|c|c|c|}
\hline Protocol & $\begin{array}{l}\text { QoS } \\
\text { metric }\end{array}$ & $\begin{array}{l}\text { Prere- } \\
\text { quisites }\end{array}$ & Description \\
\hline AQOR [73] & $\begin{array}{l}\text { Bandwidth } \\
\text { delay }\end{array}$ & $\mathrm{P} 1, \mathrm{P} 4$ & $\begin{array}{l}\text { A resource reservation-based routing and signalling } \\
\text { algorithm. AQOR includes efficient mechanisms for QoS } \\
\text { maintenance, including temporary reservation and } \\
\text { destination initiated recovery processes }\end{array}$ \\
\hline $\begin{array}{l}\text { AQuoS- } \\
\text { DeRenesse } \\
\end{array}$ & Bandwidth & $\mathrm{P} 1$ & $\begin{array}{l}\text { Solution based on a three-part architecture: resource } \\
\text { analysis, admission control and traffic differentiation. A } \\
\text { route recovery process is not defined. }\end{array}$ \\
\hline BEQR [70] & Bandwidth & $\mathrm{P} 1$ & $\begin{array}{l}\text { Two schemes: admission control and feedback. A route } \\
\text { recovery mechanism is included. However the bandwidth } \\
\text { estimation mechanism increases the overload. }\end{array}$ \\
\hline DOPA [75] & Delay & $\mathrm{P} 1$ & $\begin{array}{l}\text { It is an on-demand delay-oriented power-aware routing } \\
\text { protocol. However it lacks of re-routing mechanism. }\end{array}$ \\
\hline $\begin{array}{l}\text { qADOV } \\
{[76]}\end{array}$ & $\begin{array}{l}\text { Bandwidth } \\
\text { and delay }\end{array}$ & $\mathrm{P} 1$ & $\begin{array}{l}\text { It modifies AODV to improve its performance. The } \\
\text { bandwidth and delay are jointly considered as the route } \\
\text { selection criteria }\end{array}$ \\
\hline $\begin{array}{l}\text { Q-AODV } \\
\text { [67] }\end{array}$ & $\begin{array}{l}\text { Bandwidth } \\
\text { delay }\end{array}$ & P1, P2 & $\begin{array}{l}\text { It defines QoS extensions for AODV protocol. These } \\
\text { extensions specify the service requirements in terms of } \\
\text { bandwidth or delay. However, it does not specify how some } \\
\text { methods of the routing protocol must be implanted. }\end{array}$ \\
\hline $\begin{array}{l}\text { QMRB- } \\
\text { AODV [77] }\end{array}$ & Bandwidth & P3 & $\begin{array}{l}\text { The protocol constructs a routing backbone consisting of } \\
\text { nodes that are rich in resources. It may not allow the } \\
\text { backbone nodes to move }\end{array}$ \\
\hline $\begin{array}{l}\text { QRPAM } \\
\quad[3]\end{array}$ & $\begin{array}{l}\text { Bandwidth } \\
\text { delay }\end{array}$ & $\mathrm{P} 1, \mathrm{P} 5$ & $\begin{array}{l}\text { DSR-based protocol. This proposal is a multipath routing } \\
\text { protocol that uses a simplified QoS model. It can find three } \\
\text { paths which are selected according to the bandwidth } \\
\text { constraint as the main condition. }\end{array}$ \\
\hline $\begin{array}{l}\text { QS-AODV - } \\
\text { Zhang [78] }\end{array}$ & Bandwidth & P1, & $\begin{array}{l}\text { Based on admission control scheme. Contention between } \\
\text { packets of the same flow is not estimated. A route recovery } \\
\text { mechanism is included. Bandwidth estimation method is } \\
\text { not specified. }\end{array}$ \\
\hline RSQR [79] & $\begin{array}{l}\text { Bandwidth } \\
\text { delay }\end{array}$ & P1, P5 & $\begin{array}{l}\text { It is a protocol based on AODV. This proposal uses the route } \\
\text { stability as a metric during the route discovery to improve } \\
\text { the durability of established route. }\end{array}$ \\
\hline
\end{tabular}

Pre-requisites: P1- Homogeneous nodes P2-Resource reservation protocol, P3- Heterogeneous nodes, P4IEEE 802.11 DCF, P5- transmission range is equal to interference range.

To provide quality of service, extensions can be made in the routing table and in the packets used during the route discovery process. As described in Section 3.2, there are several QoS routing solutions based on AODV. The most important approach is called QAODV which is defined in the internet draft [67]. However, this proposal does not specify how some elements of the routing protocol must be implanted, such as: the 
methods to compute available bandwidth and the end-to-end delay, the route recovery process due to link failure and the admission control mechanism. As a consequence of these shortcomings, QAODV does not show acceptable results during overload network condition. Hence, some traffic flows can be rejected on the basis that they cannot be carried. However, maybe the source application could adjust some parameters in the coding to adapt its data rate to the network condition. The feedback scheme implemented in the routing protocol proposed in this thesis indicates to the source node about the network conditions and makes more efficient use of the available bandwidth. Moreover, a mechanism for the estimation of the available bandwidth and the route recovery process are defined.

\subsection{Routing protocol proposal: AQA-AODV}

This section describes the details of the proposed routing protocol called AQA-AODV (Adaptive QoS-Aware Ad-hoc On-demand Distance Vector), which is the main contribution of this thesis. Although AQA-AODV is based on AODV protocol, it incorporates three new elements:

1. An algorithm used for the estimation of the available bandwidth that allows nodes along the path to know their available resources (in terms of bandwidth).

2. A cross-layer mechanism to inform to the application layer the available bandwidth by which the source node can easily adapt its transmission rate.

3. A new route recovery mechanism that involved the modification of the route request and the implementation of a session cache table to store information about the established sessions and its QoS conditions.

Some changes in the format of the packets used in AODV were required to implement the above modifications. For example, a QoS extension with new fields were added to the RREQ and RREP packets to carry the information about bandwidth requirements, transmission rate and a session ID (used to identify each QoS flow). The new AQA-AODV packets are called QRREQ, QRREP and QRERR in order to empathize the QoS extension.

An important difference between the proposed protocol and other solutions based on AODV is the adaptive feedback scheme, integrated into the routing protocol, by which the source node can know and easily adapt its transmission rate according to the state of the route. For this reason, nodes along the path must know their available resources by using some algorithms.

Figure 3.2 depicts the functional block diagram of AQA-AODV. The main three elements of AQA-AODV are a bandwidth estimation module, a routing module and a route recovery module.

The first module performs the estimation of the available bandwidth and provides data feedback to the video application. HELLO packets are used in the bandwidth estimation, which is periodically executed according to the trigger of Timer module. The information about the available bandwidth is used by video application in order to tune a coding parameter to compose a video stream that can be supported by network. On the other 
hand, the routing module receives the route requests from the application and executes the route discovery routine. When a route between source and destination is established, a unique session identifier ( sid) is assigned in the Session/sid mapper. The identification data of the sessions (sid, source and destination address, QoS requirements and expiration time) are stored internally in a database, called Session Cache List. The third main module is the route recovery module, which is in charge of reestablishing the connections after a link failure, taking into account the QoS conditions of each of the sessions.

In next sub-sections, the main tasks performed by AQA-AODV are described with more details. First, the algorithms used in the bandwidth estimation phase are described. Then, it is presented a more detailed explanation of the routines involved in the route discovery phase as well as the mechanisms of the route recovery strategy.

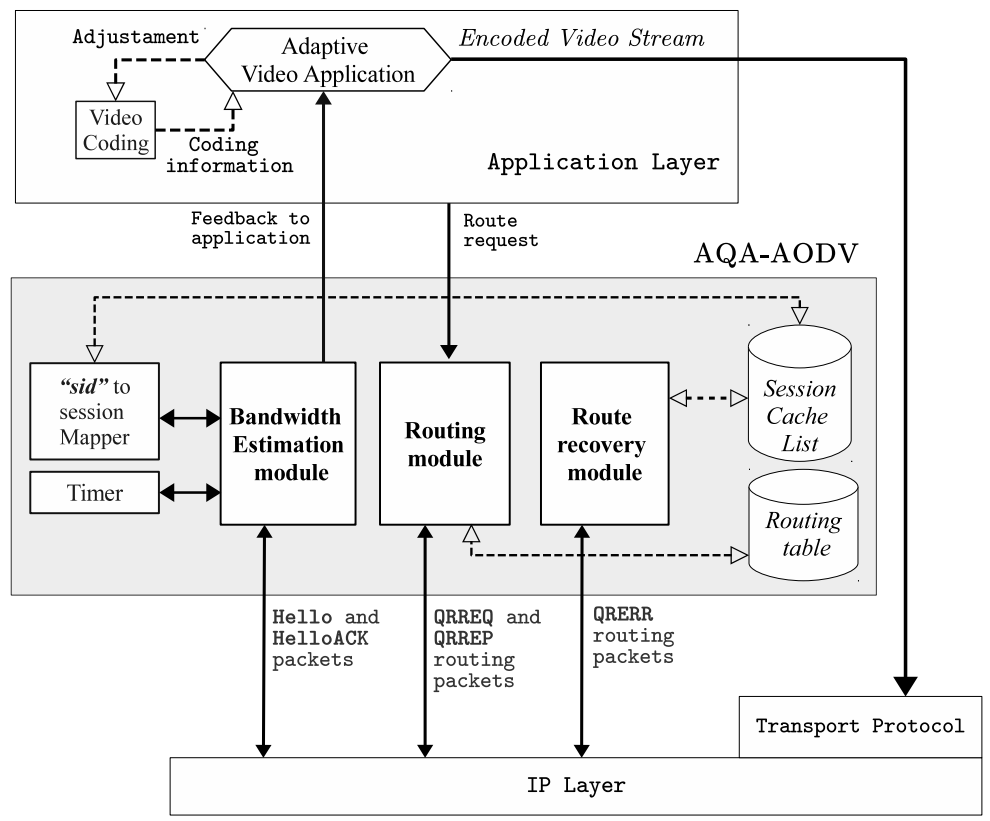

Figure 3.2. Functional block diagram of $A Q A-A O D V$

\subsubsection{Bandwidth estimation}

When an incoming flow is requesting admission in a wireless ad hoc network, the optimum transmission rate must be estimated in order to be informed to the source node. The optimum transmission rate is the data rate at which a source node sends packets achieving the highest throughput without causing congestion in the network. Therefore, this rate must be equal to or less than the available end-to-end bandwidth from the source to the destination. In wired networks, the available end-to-end bandwidth is a concave parameter, which is determined by the minimum available bandwidth of the links along the route (bottleneck bandwidth). However, this is still a 
challenging problem in wireless ad hoc networks due to many factors such as the shared nature of the wireless channel and the mobility. Moreover, a packet emission from a node has an impact on the available bandwidth of nodes located in a certain area surrounding the source node. This causes a decrease of the data rate that can support each node.

As an example, it is shown in Figure 3.3 a wireless ad hoc network where the available bandwidth (in Kbps) of each link is known. In this example, the source node (node 1) requests to send data with a rate of $120 \mathrm{Kbps}$ to node 6 . According to the values of the available bandwidth along the path, it is assumed that the source node adapts its transmission rate to $100 \mathrm{Kbps}$, which correspond to the bottleneck bandwidth. However, during transmission the maximum throughput reached in the destination node is lower than the expected value. This is caused by the mutual interference between packets of the same flow (also called "intra-flow contention"). As analysed in Chapter 2, intra-flow contention occurs when nodes along a multihop route contend among themselves for channel access to forward packets belonging to the same flow.

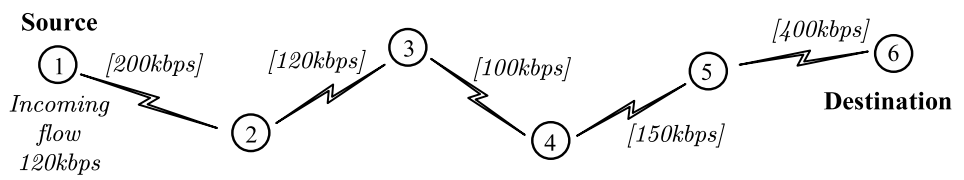

Figure 3.3. Example of wireless ad hoc network with the available bandwidth of each link

According to the previous example, two different stages can be identified in the process of feedback of the optimum data rate to the source node:

1. The local estimation of each node of its available bandwidth (BWav) in order to know the available end-to-end bandwidth along the route.

2. The prediction of the total amount of bandwidth that the new flow can consume ( $B W_{\text {consumed }}$ ) along a route of $n$ hops taking into account the "intraflow contention".

Therefore, it is proposed a new evaluation method of the available end-to-end bandwidth. The method includes performing checks on all nodes along the route in order to verify if each node could support the consumed bandwidth (BWconsumed) according to his local estimation of the available bandwidth.

The diagram shown in the Figure 3.4 describes in general form, the main tasks involved in the admission of incoming flows. The routine $P l$ is performed in each node using only its local perception and $P 2$ is executed by destination node based on the requested rate. From the local available bandwidth estimated locally in the nodes, it is determined the available end-to-end bandwidth (P3). The criteria used for accepting a new flow is shown in the decision block $D 1$. That is, if consumed bandwidth by the incoming flow is lower than the available end-to-end bandwidth, then the source node can to transmit at the requested data rate. Otherwise, the source node must adapt its data rate to the value of the available end-to-end bandwidth measured in the path. In order to ensure that all 
nodes along the route could support the new flow, the condition D1 must be checked in the intermediate nodes from source to destination node.

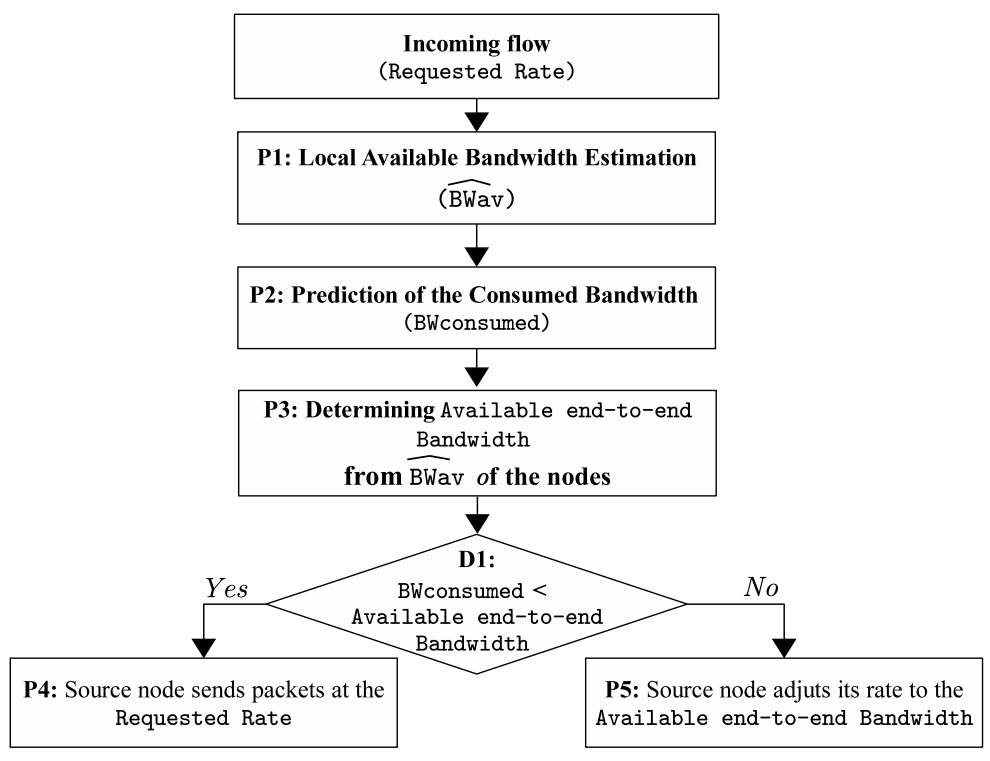

Figure 3.4. Flowchart of the tasks involved in the bandwidth estimation phase

In next subsections, it is provided a brief description about the algorithms used to estimate the Local Available Bandwidth $(B W a v)$ in each node and to predict the bandwidth to be consumed ( $B W_{\text {consumed }}$ ) by the requesting flow.

\subsection{1.a. Estimation of local available bandwidth in AQA-AODV}

The algorithm for estimating local available bandwidth consists of two steps:

1. Step 1: each node estimates its local available bandwidth.

2. Step 2: the nodes calculate a weighted average of the most recent values in order to obtain a final estimation of the local available bandwidth.

In the first step, to estimate the local available bandwidth (remaining bandwidth) between two neighbour nodes, it is assumed the Definition 1.

Definition 1: The local available bandwidth between two nodes is defined as the maximum throughput that can be transmitted between these two nodes without negatively affecting any ongoing flow in the network (permissible throughput).

The Definition 1 should not be confused with the classical definition that considers the available bandwidth as the unused capacity of the link. The problem of the estimation of the local available bandwidth can be, therefore, re-formulated as the determination of the maximum throughput that can be used for the data transmission between two nodes in the current time. This assumption is used in other works, such as [80], [81], [8] and [82]. 
In the algorithm proposed in this thesis, a given node can estimate its permissible throughput to each neighbour by the Equation (1). Where $S$ is the size (in terms of bits) of the packet sent from the node to its neighbour. $T s$ is the time-stamp when the packet is ready at the MAC layer, and Tr is the time-stamp when an ACK has been received (see Figure 3.5).

$$
T h_{\text {packet }}=\frac{S}{T r-T S}
$$

Several methods have been proposed in order to measure the parameters of Equation (1). For example, Munaretto and Fonseca [83] propose to use a train of probe packets in order to observe the network performance. However, this technique introduces additional traffic into the network. Another method was used by Shah et al. [84] who obtained the parameters $T s$, $T r$ and $S$ by modifying the MAC layer. However, it requires that changes must be performed to the firmware of standard 802.11 hardware.

It is proposed a different mechanism in the measurement of the parameters of the Equation (1). This allows for a more accurate estimation of the local available bandwidth as well as an implementation easier. In this approach, each node in the network can estimate the permissible throughput to its neighbours by using existing HELLO packets, which are used to discover neighbours in AODV. The proposed algorithm has two main advantages. First, it can be implemented in network layer, as opposed to firmware. Second, more overload to the network is avoided due to the use of the HELLO packets of AODV.

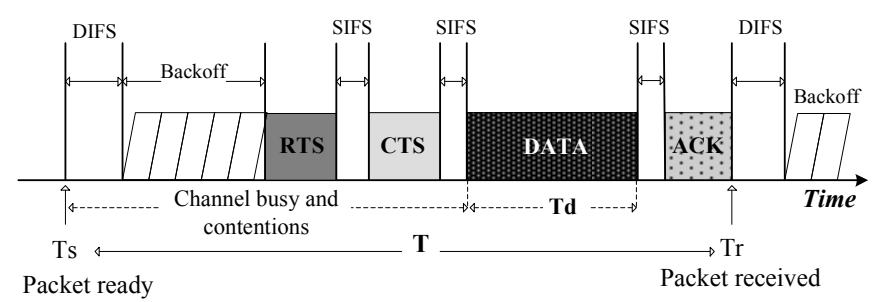

Figure 3.5. Transmission of a single packet in IEEE 802.11 DCF MAC protocol

The implementation of the local bandwidth estimation algorithm can be explained with more details as follows.

1. Let $i$ be a node and $j$ its neighbour.

2. To estimate the permissible throughput at the node $i$, a HELLO packet is sent from node $i$ to $j$.

3. The time-stamp (TS) when the packet is ready to be sent is recorded.

4. When node $j$ receives the HELLO packet, it sends back to node $i$ a HELLOAck packet carrying the time-stamp Ts.

5. Finally, the time-stamp $T r$ is recorded in node $i$ when the HELLOAck packet is received. 
HELLOAck packet is an additional packet created to make more accurate the measurement of the throughput since it does not depend of the throughput seen by only one packet. The proposed available bandwidth estimation, based on the exchange of HELLO and HELLOAck packets, includes the channel busy, the contention time, the MAC overhead and the current time used for data transmission. Therefore, in order to understand what the algorithm includes, the Equation (1) can be rewritten using the explicit form shown in Equation (2). This equation is expressed in terms of $\mathrm{BW}_{\mathrm{av}}$ according to the Definition 1.

$$
B W_{a v}=\frac{R T S+C T S+\text { Hello }+A C K+R T S+C T S+\text { HelloAck }}{T r-T S}
$$

Where,

Tr-Ts $=\mathrm{T}_{\mathrm{Q}}+\left(\mathrm{T}_{\text {Hello }}+\mathrm{T}_{\text {Hello }}+\mathrm{T}_{\mathrm{CA}}+\mathrm{T}_{\mathrm{OH}}\right) \times \mathrm{R}+\sum_{1}^{\mathrm{R}} \mathrm{B}_{\mathrm{T}}$

According to the Equation (2), the packets sent and received during the time interval $T r$ $T s$ involve not only the HELLO packets, but also the MAC control packets (RTS, CTS and $A C K$ ). The period of time $\mathrm{Tr}$ - $T$ s takes into account the time used for transmitting HELLO and HELLOAck packets ( $T_{\text {Hello }}, T_{\text {HelloAck }}$ ), the queuing time $T_{Q}$, collision avoidance phase time (SIFS, DIFS) $T_{C A}$, the control overhead time (RTS, CTS) $T_{O H}$ and back-off time $B_{T}$ for $R$ retransmissions.

The measurement mechanism captures the effect of channel contention, fading and interference errors. Since, if contention is high, $T r-T s$ will increase, and therefore, the available bandwidth will decrease. This mechanism also captures the effect of physical errors because if the RTS or HELLO packets are affected by channel errors, they have to be re-transmitted. Thus, the interval $T r$ - $T s$ is increased and correspondingly the available bandwidth perceived by the node is decreased.

The rate of the estimation of the local available bandwidth at each node is the interval of time $\Delta t$, which corresponds to the period between HELLO messages. The accuracy of the bandwidth calculation depends on the value of $\Delta t$. A small interval increases the precision of the estimation but increases the overhead. Therefore, there is a trade off between the duration of the interval and the bandwidth usage. However, $\Delta t$ should be small enough to allow fast reactions to changes of the load and the topology. In addition, the periodic nature of the HELLO generation has the disadvantage that it increases the risk of simultaneous transmissions resulting in collisions. Therefore, as HELLO interval $(\Delta t)$ were chosen a random value between 1 and 1.5 seconds, which is the recommended value in [46]. This decreases the probability of simultaneous transmissions.

In the second step of the estimation of the local available bandwidth, each node calculates a weighted average of the most recent values of the Local Available Bandwidth in order to obtain a stable and yet responsive system of estimation (Equation (3)).

$$
\widehat{B W}_{a v}\left(t_{i}\right)= \begin{cases}\alpha B W_{a v}\left(t_{i}\right)+(1-\alpha) \times \widehat{B W}_{a v}\left(t_{i}-1\right) & t>0 \\ B W_{a v}\left(t_{o}\right) & t=0\end{cases}
$$


Where $\widehat{B W}_{a v}\left(t_{i}\right)$ is the new weighted average of the local available bandwidth, $B W_{a v}\left(\mathrm{t}_{\mathrm{i}}\right)$ is the actual measurement of the local available bandwidth in the time period $t_{i}$, $\widehat{B W}_{a v}\left(t_{i-1}\right)$ is the weighted average calculated in the previous time period $t_{i-1}$, and $B W_{a v}\left(\mathrm{t}_{\mathrm{o}}\right)$ represents the initial measurement of the local available bandwidth. A value of $\alpha=0.8$ was used in the algorithm, which were determined by a few empirical trials. This value of $\alpha$ allows the algorithm a fast reaction to changes of the network condition.

In summary, when a given node needs to estimate the local available bandwidth it calculates the permissible throughput with its neighbours in the current time, using exchange of HELLO packets. The measured throughput allows the node to infer the value of the available bandwidth at that node. Finally, in order to obtain a stable and yet responsive system of estimation, each node calculates a weighted average of the Local Available Bandwidth $\left(\widehat{B W}_{a v}\right)$. Results show that this method is the most conservative of all the methods discussed and it gives the more accurate results. Figure 3.6 illustrates how to use the HELLO and HELLOAck packets in the estimation of the permissible throughput.

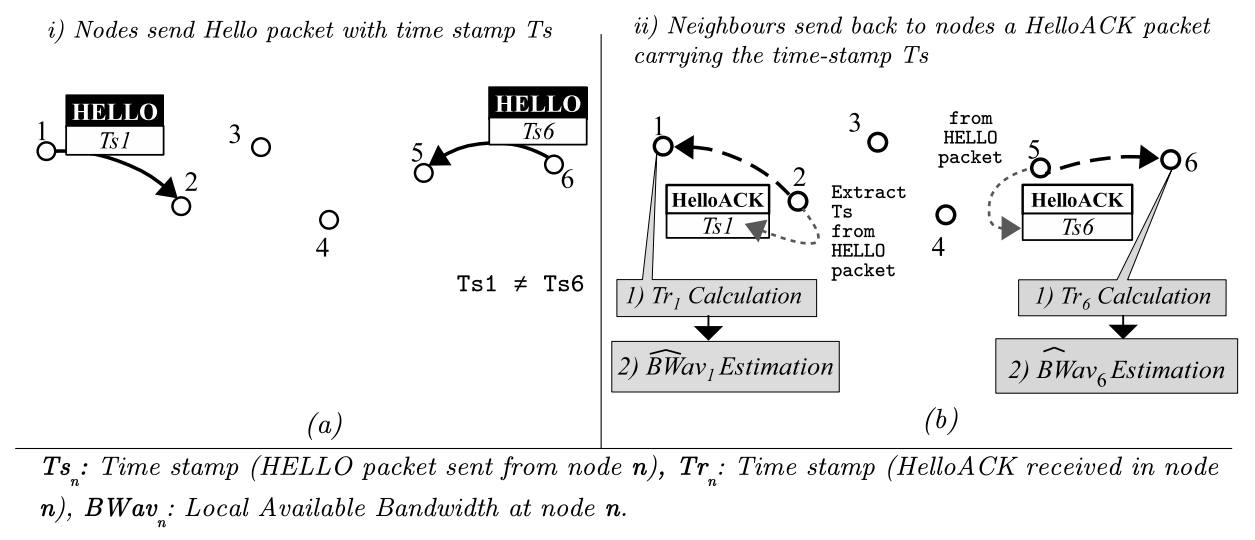

Figure 3.6. Using HELLOs packets in the bandwidth estimation

\subsection{1.b. Prediction of the consumed bandwidth along the path}

The second stage of the bandwidth estimation method consists of estimating the consumed bandwidth along the path to check if all nodes along the route could support the bandwidth requested by the source. In order to calculate this consumed bandwidth it is necessary to take into account the mutual interference between packets of the same flow. This sub-phase is executed only by the destination node when a new flow starts the route discovery process.

The method for calculating the intra-flow contention is based on the parameter called Contention Count (CC) [74]. The value of this parameter will help to calculate the consumed bandwidth for the route during a transmission. The proposed method, assumes that interference range is twice of the transmission range. It means that the nodes that are one hop or two hops away from the transmitter will get the interference 
and cannot use the channel. In other words, this method only considers the interference coming from nodes that are one hop and two hops away.

The contention count is calculated as shown Equation (4).

$$
C C=h_{\text {req }}+h_{\text {rep }}
$$

The calculation of the $h_{r e q}$ and $h_{r e p}$ parameters must satisfy the next two conditions:

1. $\quad$ if $h_{r e q}>2$ then $h_{r e q}=2$

2. if $h_{\text {rep }}>3$ then $h_{\text {rep }}=3$

The $h_{r e q}$ and $h_{r e p}$ parameters mean the number of hops from one node to the source and destination nodes, respectively. They can be directly obtained from the Hop Count field of the RREQ and RREP packets of AODV.

Figure 3.7 shows an example in order to explain the calculation of $C C$ in a clear way. In the example, it is assumed that there is a flow intended to be sent from node 1 to node 6. Taking the node $3, h_{r e q}$ is 2 and $h_{\text {rep }}$ is 3 , then $C C=2+3=5$ according to the Equation (4).

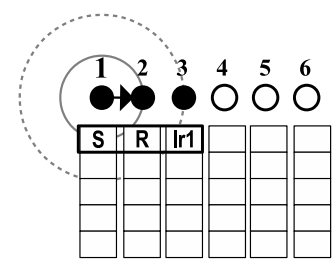

(a)

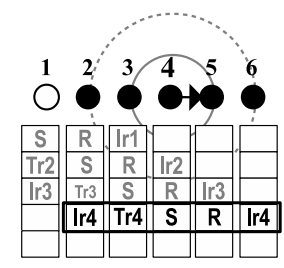

(d)

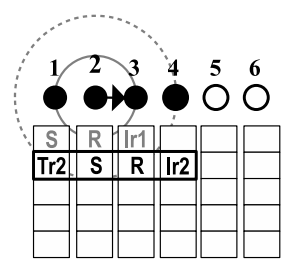

(b)

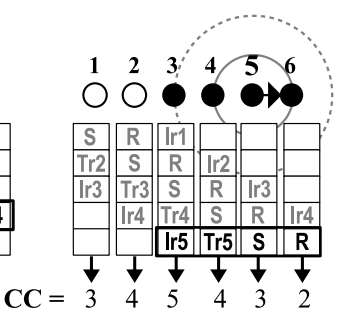

(e)

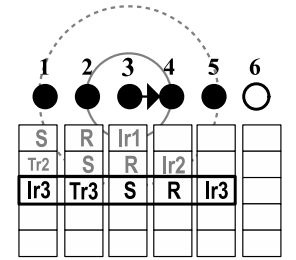

(c)

Figure 3.7. Example of Intra-flow contention calculation

Figure 3.7 shows how each node calculates its CC parameter. Irn means that the node is in the interference range of node $n$ that is transmitting. Similarly, Trn means that the node is in the interference range of node $n$ that is transmitting. For example, when node 3 is transmitting packets to node 4 , node 1,2 and 5 will get the interference and the channel for these nodes should be set as busy since they share channel with node 3 . This example illustrates how the maximum CC value is obtained in the node 3 , which causes a bandwidth bottleneck at that node. In Figure 3.7 it can be seen that for a route with 5 or more hops the CCmax has a fixed value of 5 and for routes with less than 5 hops, CCmax is equal to the number of hops. 
After estimating CCmax, destination node could calculate the consumed bandwidth as shown in the Equation (5). Where reqBW is the bandwidth requested by the source node, CCmax indicates the maximum value of the contention count in the route and $B W_{\text {consumed }}$ means the consumed bandwidth expected along the route if a flow is transmitted through path with data rate equal to reqBW.

$$
B W_{\text {consumed }}=\text { CCmax } \times \text { reqBW }
$$

In summary, when a destination node receives a $Q R R E Q$ packet, it calculates the CCmax and the $B W_{\text {consumed }}$ according to the Equation (5). Subsequently, the destination node compares the $B W_{\text {consumed }}$ with the last value of its local available bandwidth $\left(\widehat{B W}_{a v}\right)$, which has been calculated using the Equation (3). If $B W_{\text {consumed }}$ is less than $\widehat{B W}_{a v}$ in the destination node, it informs the source node that the transmission rate must be equal to the requested bandwidth (reqBW). Otherwise, if the $\widehat{B W}_{a v}$ in the destination node is less than the value of $B W_{\text {consumed, }}$ the source must adjust its transmission rate to $\widehat{B W}_{a v} / C C_{\max }$.

Considering again the network described in Figure 3.3, an example of the interaction of the algorithms used in the bandwidth estimation phase of the proposed protocol could be described as follows. Suppose that node 1 requests to transmit with a data rate of 120 Kbps $(r e q B W)$ to node 6 . In this case, the CCmax calculated will be 5, since the node 3 is at a distance of 2 hops from source node and of 3 hops from the destination. Therefore, the value of $B W_{\text {consumed }}$ calculated in the destination node will be $600 \mathrm{Kbps}(120 \mathrm{Kbps}$ $\mathrm{x} 5)$. Assuming that the local available bandwidth $\left(\widehat{B W}_{a v}\right)$ in the node 6 is $400 \mathrm{Kbps}$, it can be seen that the consumed bandwidth ( $B W_{\text {consumed }}$ ) is higher than the $\widehat{B W}_{a v}$. This means that if the source node transmits to $120 \mathrm{Kbps}$ the destination could not support such rate. Therefore, the destination calculates a new rate according to its local available bandwidth. Then the destination node notifies the source that the transmission rate must be $80 \mathrm{Kbps}\left(\widehat{B W}_{a v} / C C_{\max }=400 \mathrm{Kbps} / 5\right)$. This data rate is lower than the rate of 100 Kbps reported to the source in the previous example described in the section 3.4.1. This difference is due to the fact that the mutual interference between packets of the same flow were introduced in the estimation of the consumed bandwidth.

In next section, it is explained the integration of the bandwidth estimation algorithms in the route discovery process.

\subsubsection{Route discovery in $A Q A-A O D V$}

The route discovery process of AQA-AODV was designed so that route entry is created based on the application requirements, i.e. the application indicates in the request message the minimal bandwidth that must be guaranteed. If network cannot support this requirement, the application can adjust its data rate according to the value received from the network.

For route discovery, if a source node requested a route to a destination node with specific bandwidth requirements, it broadcasts a RREQ packet with the QoS extension (QRREQ) to its neighbour nodes (Figure 3.8a). This packet includes a Session ID, which is used with the source address to uniquely identify each traffic flow. The Session ID is 
generated by a counter which is incremented by a node each time it constructs a new QRREQ. The session id counter, as well as broadcast id counter used in AODV, is a separate counter that is maintained by each node.

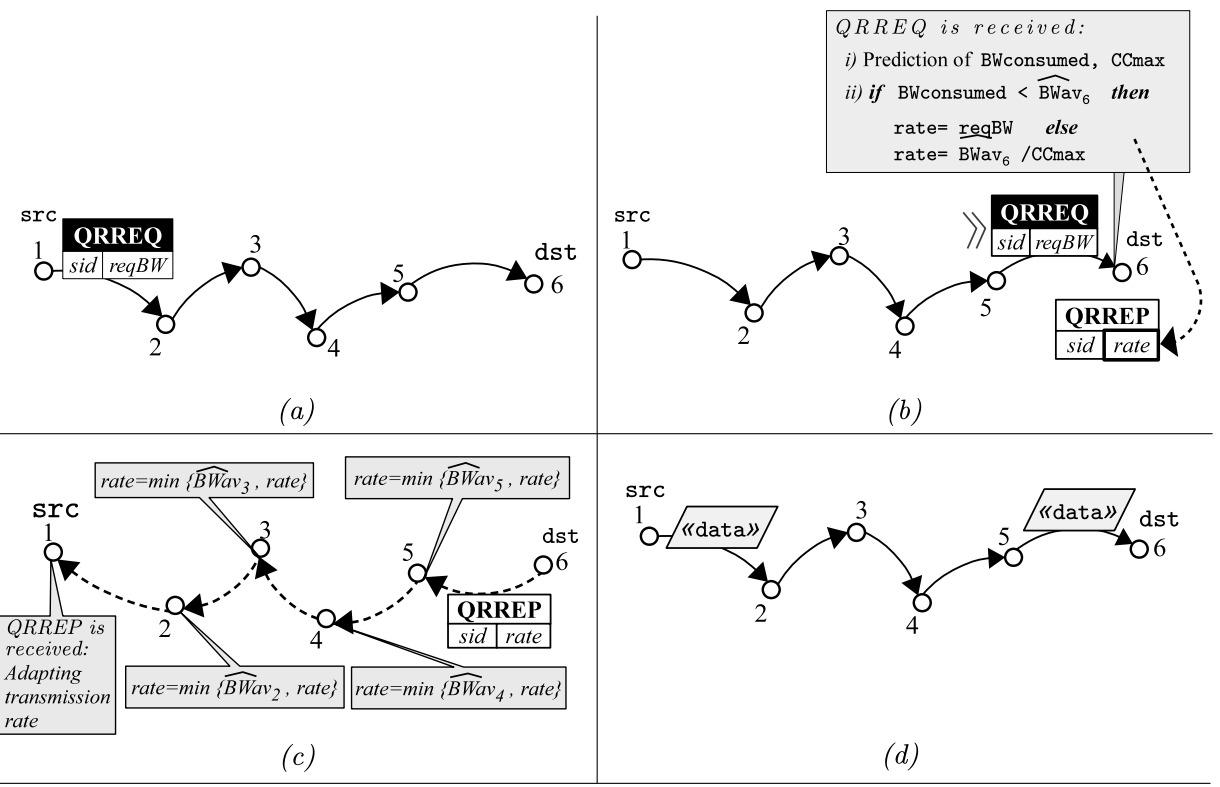

sid-Session ID, BWav $\boldsymbol{n}^{-}$Local Available Bandwidth at node $\boldsymbol{n}$, reqBW- Requested bandwidth, BWconsumedConsumed bandwidth along the route, rate- Suggested Transmission Rate, QRREQ and QRREP- modified $A O D V$ packets with $Q o S$ extension (sid, rate, reqBW).

Figure 3.8. Example of the route discovery phase in $A Q A-A O D V$

When a node receives a $Q R R E Q$ packet, a reverse route entry is created with the Session $I D$, and the QRREQ packet is rebroadcasted. This process continues until the QRREQ packet reaches the destination node (Figure 3.8b). In AODV, a RREP packet can be created by the destination node or an intermediate node with a "fresh enough" route to the destination. However, only the destination will be able to send the route reply packet $(Q R R E P)$ in AQA-AODV. This will ensure that all nodes in the selected route satisfy the bandwidth constraints. When the destination node receives a QRREQ packet, if it is a new request, a reverse route entry for the new session is created. Before sending the QRREP to the source, local available bandwidth $\left(\widehat{B W}_{a v}\right)$ is checked and the consumed bandwidth along the path ( $B W_{\text {consumed }}$ ) is estimated. Finally, the QRREP will be transmitted to the source with a modified header that includes the minimum value between the bandwidth requested by the source $(r e q B W)$ and the maximum bandwidth that all nodes along the route could support taking into account the intra-flow contention, (i.e. $\widehat{B W}_{a v} / C C_{\max }$ ) such as is described in section 3.4.1.b. Once an intermediate node receives the QRREP packet, it compares its local available bandwidth with the bandwidth indicated in the QRREP. If its local available bandwidth is lower, it replaces the value stored in the min-bandwidth field of $Q R R E P$, with the value of its local available bandwidth. Otherwise, the node forwards the QRREP (Figure 3.8c). This 
procedure will ensure that the source knows the minimum bandwidth along the path, which will be the maximum rate that it may transmit. Once the source node receives the QRREP packet, it adjusts its transmission rate according to the value of the field rate in QRREP and then the transmission of the data packets is started (see Figure 3.8d).

\subsubsection{Route recovery mechanisms}

On account of changes in topology caused by the mobility of the nodes and the condition of having a shared physical channel, the communications inside mobile ad hoc networks usually show frequent disruptions. For this reason, it is necessary to implement a route recovery mechanism. This mechanism not only has to re-establish the connections but also take into account the conditions of QoS that have been established during the route discovery phase.

The route recovery mechanism of AQA-AODV detects the connection losses in a route when a host does not receive a HELLO message from a neighbour during an interval of time. The HELLO messages may not be received for three main reasons:

1. Case 1: there is total connectivity but some of the HELLO messages are lost because of congestion.

2. Case 2: the neighbour node is no longer available because it is out of transmission range and the node should look for a new path to the destination.

3. Case 3: the destination node is no longer available in the ad hoc network and the connection recovery is not possible.

The route recovery mechanism implemented in AQA-AODV, perfectly works in any of the two previous cases in which connection recovery is possible. To explain the functionality of the proposed route recovery mechanism in detail, two examples (one for each case: case 1 and case 2) are shown in Figure 3.9 and Figure 3.10.

The example showed in Figure 3.9 consists of a network with four nodes in which each node is inside the transmission range of its one hop neighbors and inside the interference range of its two hop neighbors. Node 1 (source node) broadcast a QRREQ message to obtain a route to node 4 with a transmission rate of $1 \mathrm{Mbps}$. When the destination node checks that the maximum transmission rate available is $0.5 \mathrm{Mbps}$, it sends the information to the source using a QRREP message. During the backward process of the $Q R R E P$, each node checks its own available bandwidth and compares it to the value included in the QRREP message. This process has been described in previous Section 3.4.2, which is referred to hereafter as standard procedure of route discovery. Moreover, each node adds a register in the session cache list associated with a session identifier ( $s i d$ ) and an expiration time (Expiration Time) with the aim of erasing the old registers (see Figure 3.9a).

Once the route from node 1 to node 4 is defined, data packets are sent through the network. Each time a node gets a data packet related to that session, it updates the expiration time of the registers, avoiding the elimination of the register and keeping the session alive (See Figure 3.9b). When some of the HELLO messages sent by node 4 are lost due to congestion, node 3 detects a link failure and it sends an error message 
$(Q R E R R)$ to the source, including the affected session identifier (esid, Error Session ID) (See Figure 3.9c). When node 1 receives the QRERR message, it queries its session cache list using the session identifier received in the QRERR message (esid). Therefore, the source sends a $Q R R E Q$ message which includes the required bandwidth, the actual data rate and the session identifier (see Figure 3.9d).

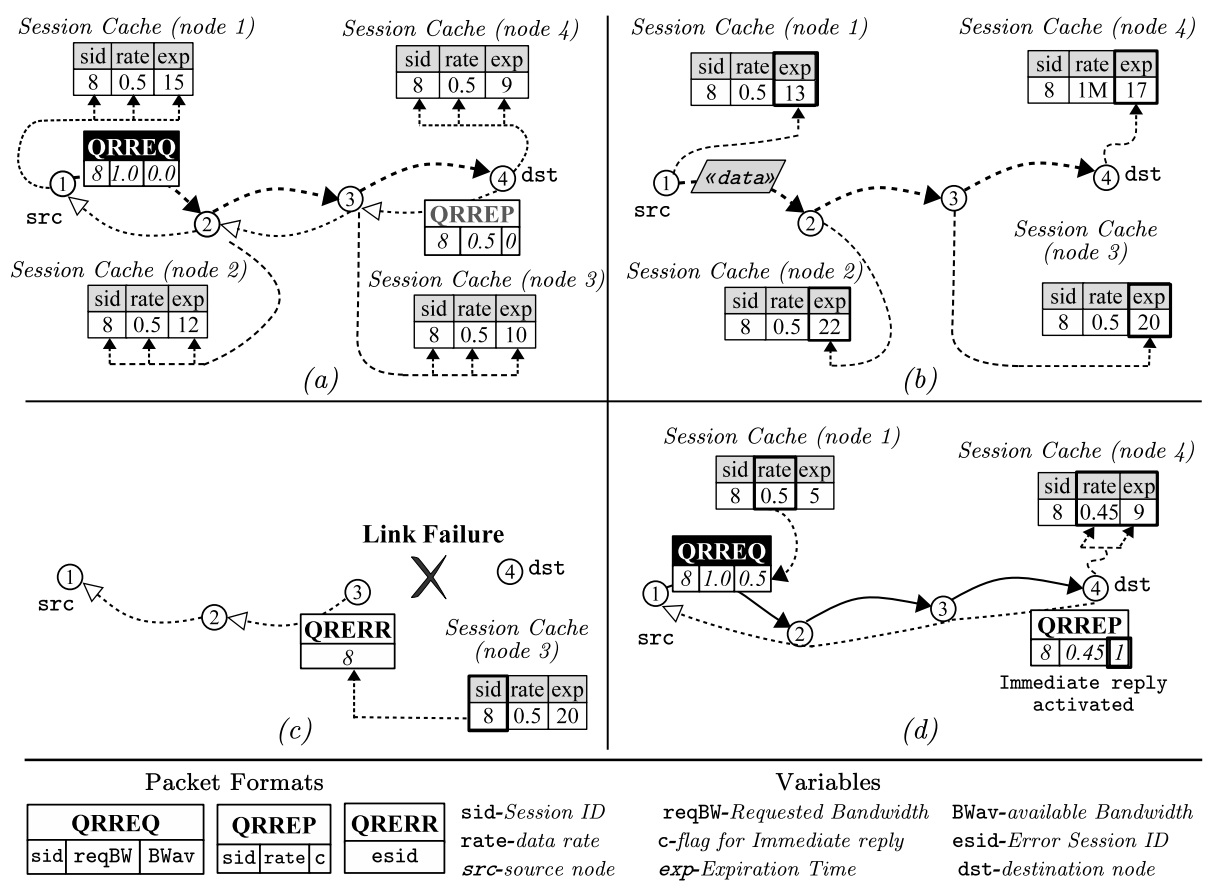

Figure 3.9. Example of route recovery mechanism. Case 1

When the destination node receives the QRREQ message it checks if it has a register with the same sid as the one sent by the source in the QRREQ. If it does have one, the destination creates a QRREP message with the same session identifier, the supported maximum data rate - this rate may be different from the original one - and an immediate reply flag ( $c=1$ immediate reply, $c=0$ standard reply). The immediate reply flag warms the intermediate nodes not to execute the standard procedure to verify the available bandwidth but send the QRREP message directly to the next hop back to the source (see Figure $3.9 \mathrm{~d}$ ). As a conclusion, the route recovery mechanism tries to re-establish connection to destination with the QoS conditions that had been negotiated during the initial route discovery phase.

The example shown in Figure 3.10, have the same conditions as in the previous example. However, a new node (node 5) has been added and it does not take part in the present route between nodes 1 and 4 . In Figure 3.10a, it can be seen the information of the previous session established in the ad hoc network, using the standard procedure mentioned before. In this example, node 4 is moving in the opposite direction of node 3 and; therefore, at some point a link failure will occur. The link failure between nodes 3 
and 4 will be informed to source node using a QRERR message (Figure 3.10b. Nevertheless, now it is possible to achieve the destination node (node 4) through node 5 , which is inside the transmission range of nodes 3 and 4 .

When node 1 receives the error message, it sends a QRREQ message and node 5, after processing the message, without finding a register associated to a session identifier (sid), proceeds to generate a new sid (Figure 3.10c). This makes the difference with the example shown in Figure 3.9. For this reason, node 4 does not take into consideration the information of the previous session and it analyses the route request in the standard way. Therefore, it calculates the available bandwidth again and compares it with the bandwidth requested by the source $(1 \mathrm{Mbps})$. The response to the route request is sent to the source through the intermediate nodes using the standard way. These nodes create a new register in session cache and check if they have enough bandwidth to transmit the traffic (Figure 3.10c).

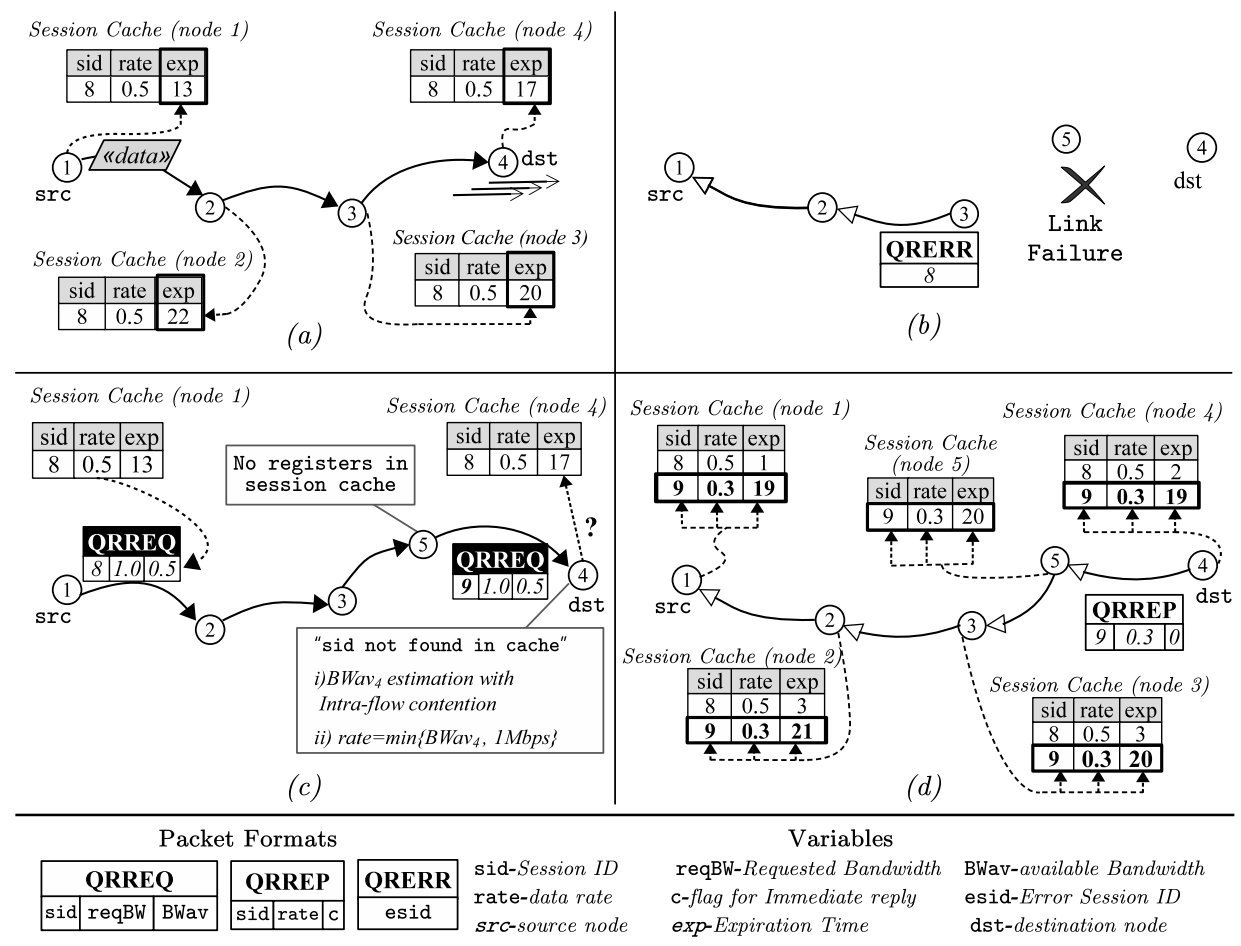

Figure 3.10. Example of route recovery mechanism. Case 2

Once the source receives the QRREP message, it adapts its transmission rate according to the available bandwidth calculated in the route recovery mechanism, and it starts sending packets to the destination. Registers in the session cache in each node are erased when the time-out expires. 


\subsection{Performance Evaluation}

The performance analysis of the proposed protocol is presented in this section. Through an extensive set of simulations, AQA-AODV is compared with AODV and the implementation of QAODV conducted by Liu et al. in [69]. QAODV was selected for performance comparison with AQA-AODV, mainly because among the recent related routing solutions, it is the closest protocol to AQA-AODV. Additionally, the performance of AODV is plotted in the graphs in order to emphasize the performance improvements regarding the typical routing protocols.

The main purpose of the simulation study is to evaluate the performance of the proposed QoS routing protocol by comparing it with conventional AODV and with a QoS routing protocol like QAODV. Later in Chapter 4, new simulation tests will be discussed in order to demonstrate the effectiveness of AQA-AODV for supporting adaptive video transmission over MANETs.

\subsubsection{The Simulation Environment}

Network Simulator 2 (NS-2) [85] has been used to test the performance of the proposed routing protocol. NS-2 contains the IEEE802.11 protocol in the MAC layer working in the distributed coordination function (DCF) mode with a channel data rate of $2 \mathrm{Mbps}$. The radio propagation model is Two Ray Ground and queue type is Drop Tail with maximum length of 50 packets. The transmission range and interference range are $250 \mathrm{~m}$ and 550 $m$ respectively. All traffic flows are Constant Bit Rate (CBR) streams over UDP with a packet size of 1000 bytes. For the first two groups of simulations, the source application used is a modified version of the CBR traffic source of NS-2, which can automatically adapt its transmission rate according the conditions presented during the simulation.

Three simulation scenarios were used in the performance evaluation. The first two scenarios were used to provide a clear exposition of the operation of the routing protocol and the third scenario was a more realistic scenario of 30 mobile nodes.

In these scenarios, the performance of AQA-AODV was evaluated measuring five metrics:

1. Packet loss rate, which is defined as the percentage of dropped packets against total packets sent.

2. End-to-end packet delay, which involves all the delay suffered by a packet that does reach the destination, including queuing, propagation and transfer time.

3. Throughput achieved along the route.

4. Number of link failures, which includes all the connection losses presented along the route.

5. Connection Setup Latency (CSL), which is the latency incurred in establishing new connection from source to destination after the previous connection is lost. This metric includes route break detection time and recovery time. 
For each type of scenario, each simulation was run for 15 times (with random scenarios with different seeds) to take average values in the measured performance metrics. The results are obtained with a confidence level of $95 \%$.

\subsubsection{Sample Network: static linear topology with 7 nodes}

The first scenario is illustrated in Figure 3.11 and consists of seven nodes placed in a linear topology in order to observe the performance of the protocol in a deterministic environment. In this scenario node 1 is the source of data traffic, which tries to send packets with a rate from 0.1 to $1.3 \mathrm{Mbps}$ to the node 7 (traffic sink).

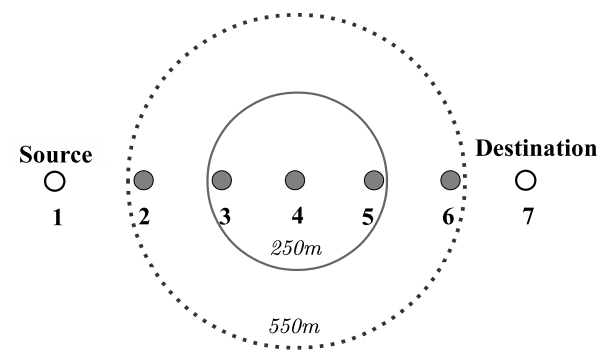

Figure 3.11. Linear topology of 7 nodes

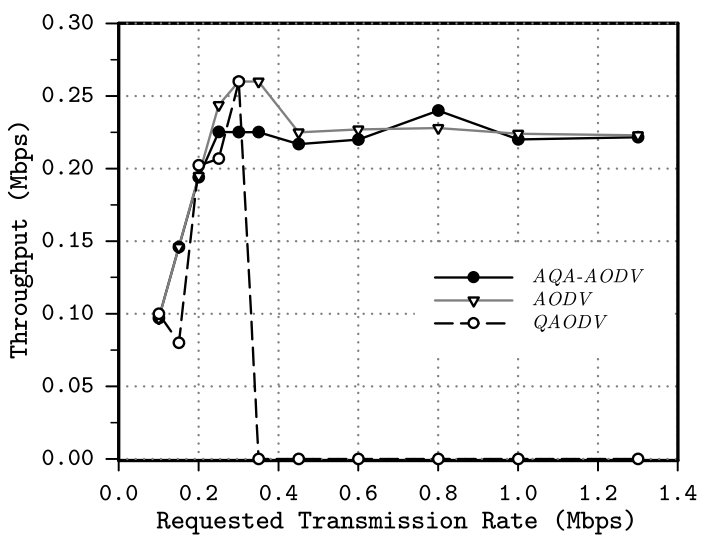

Figure 3.12. Throughput delivered by a 7-node chain with different send rate

As seen in Figure 3.12, using AQA-AODV the source sends packets with a rate equal to the requested bandwidth. This is due to fact that the bandwidth requested by the source node can be supported along the route (i.e. rates from 0.1 to $0.2 \mathrm{Mbps}$ ). On the other hand, when the route cannot support efficiently the requested data rate by the source, it adjusts its rate to the maximum rate that the chain can support. As mentioned in Chapter 2, the effective available bandwidth is reduced due to the overhead of MAC layer and the intraflow contention (CCmax). More specifically, in a chain of 7 nodes with a wireless channel capacity of $2 \mathrm{Mbps}$, the maximum throughput that one flow could reach up is about $0.23 \mathrm{Mbps}$. This value indicates a reduction of $30 \%$ due to MAC overhead 
and a reduction of $60 \%$ due to intraflow contention, when the number of hops between source and destination node is 6 or more.

Using AODV, the source does not know the available bandwidth and sends packets with data rate equal to the requested rate by the application without adaptation. These packets cannot be efficiently forwarded along the path and, therefore, loss and delay are increased significantly as the traffic increases. This means that node 1 could inject packets with a data rate greater than the forwarding rate of subsequent nodes. This fact causes an increment of the lost packets in the nodes 1, 2 and 3. Likewise, the packet losses are increased significantly when the maximum throughput that can reach a chain of 7 nodes is slightly exceeded by the source. In the case of QAODV, the transmission is established while the requested data rate by source node is less than the data rate supported by the network. Because of this controlled-access scheme of QAODV, the points on the curve for above $0.35 \mathrm{Mbps}$ in the figures, does not exist.

On the other hand, AQA-AODV performance is greatly improved in terms of end-to-end delay and packet loss, compared to AODV and QAODV results. This proves an efficient use of the available bandwidth and an accurate consumed bandwidth prediction for the adaptive feedback scheme (see Figure 3.12). The results in terms of packet loss and delay are shown in Figure 3.13 and Figure 3.14, respectively.

Results show that feedback scheme allows the source application to adapt its transmission rate close to the optimal rate that can be supported for a 7-node chain. Consequently, the total drop rate will keep below $9 \%$ and the end-to-end delay below $0.4 \mathrm{~s}$.

In figures, label "rejected" was added on the curves of QAODV in order to denote that the traffic flow is rejected by the admission control system when the route cannot support the requirements of the flow.

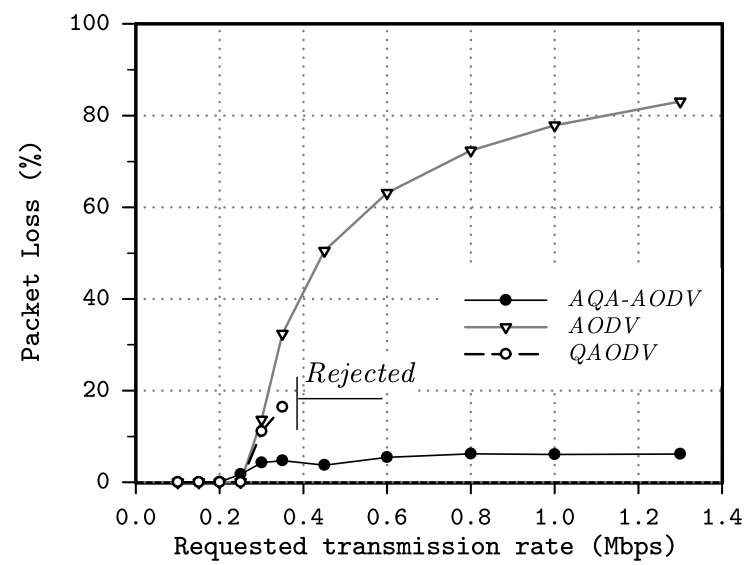

Figure 3.13. Packet loss in 7-node chain with different transmission rate 


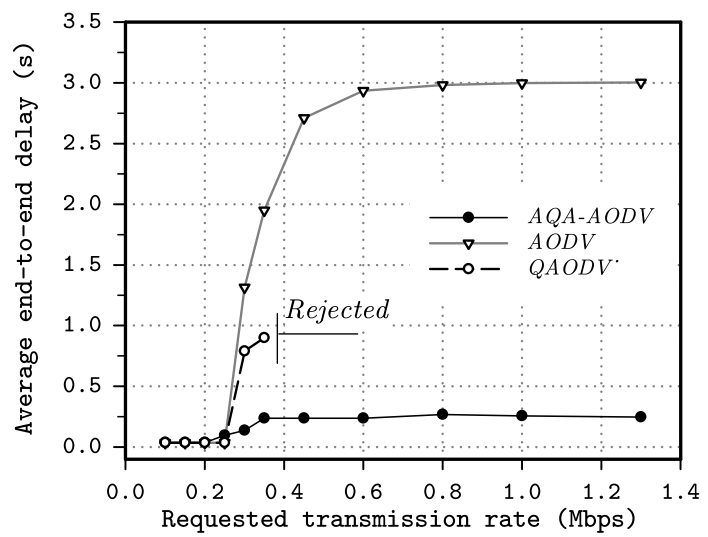

Figure 3.14. Average end-to-end delay in 7-node chain with different transmission rate

Figure 3.15 shows the number of link failures and the Connection Setup Latency (CSL). When the maximum throughput that can be reached by a 7 -nodes chain (about 0.20 Mbps) is exceeded by the source, the number of link failures drastically increases for AODV. With QAODV, its bandwidth estimation algorithm allows the network to accept traffic flows above $0.23 \mathrm{Mpbs}$ but below $0.35 \mathrm{Mbps}$. In the case of AQA-AODV, the adaptive feedback scheme lets the source to adapt the transmission rate, so losses caused by congestion are mostly avoided and it has a low number of link failures. Regarding the duration of these link failures, CSL values are lower for AQA-AODV protocol in comparison with AODV and QAODV and vary from $0.01 \mathrm{~s}$ to $1.5 \mathrm{~s}$. Therefore, AQA-AODV could be more efficient in real-time data transmissions (e.g. video streaming).

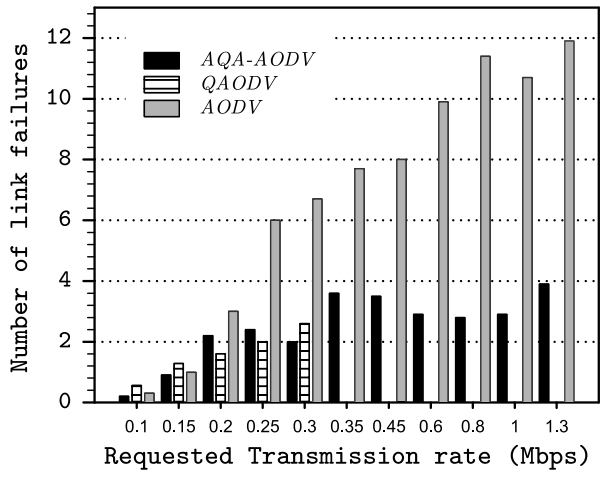

(a)

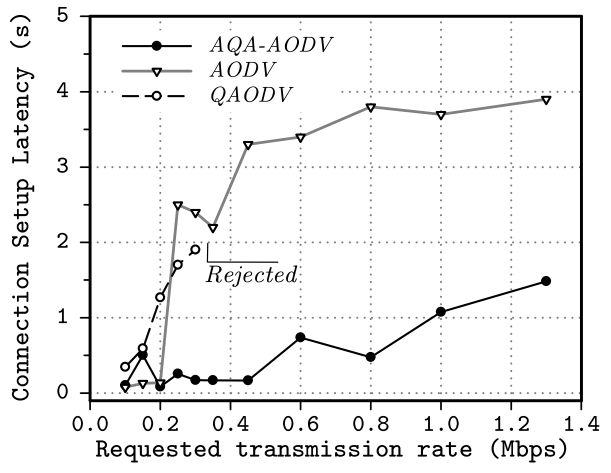

(b)

Figure 3.15. Number of link failures (a) and Connection Setup Latency (b) in linear scenario of 7 nodes

\subsubsection{Scenario 2: Static linear topology with variable length}

The second scenario is illustrated in Figure 3.16 and consists of a variable number of nodes placed in a linear topology. Node 1 is the source of data traffic and the last node 
in the chain is the traffic sink. In this scenario, the performance of AQA-AODV was tested as function of the number of hops on the path. Initially the source requested a transmission rate of $0.35 \mathrm{Mbps}$, which be maintained constant when AODV and QAODV are used, but can be changed by the source when AQA-AODV is used in the network because of the adaptive feedback scheme.

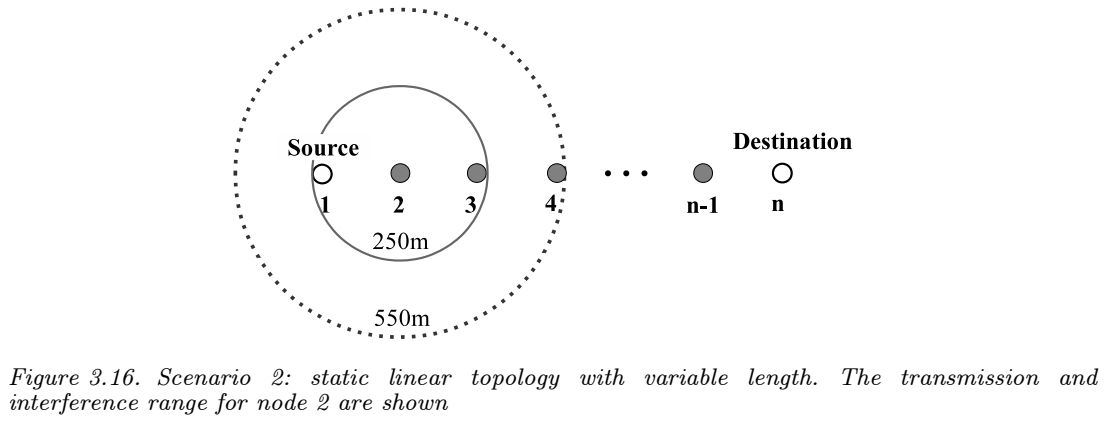

As seen in Figure 3.17 the total network throughput achieved with AQA-AODV is very close to throughput achieved using AODV. However, with AODV the source does not know the available bandwidth and it injects packets to the network with data rate equal to the requested rate $(0.35 \mathrm{Mbps})$ by the application without adaptation. These packets cannot be efficiently forwarded along the path and, therefore, the network congestion and packet loss are significantly increased. which cannot be efficiently supported in a route with more than 5 nodes. This fact leads to network congestion and excessive packet loss. On the other hand, with AQA-AODV helps avoid the congestion transmitting to the optimum data rate.

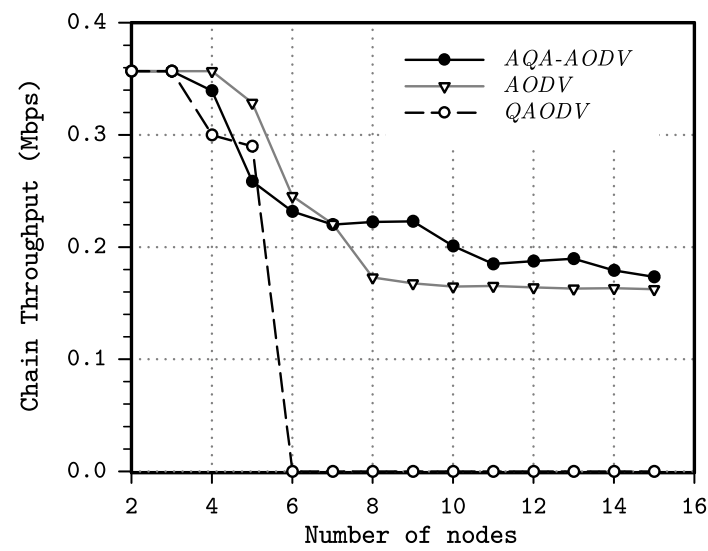

Figure 3.17. Throughput achieved along the path as a function of the chain length

In general terms, with AODV the source sends more packets to the network but a high percentage of these packets are eliminated due to the congestion. In contrast, using AQA the source sends packets to the network a lower rate and therefore the network congestion is significantly reduced. In other words, the adaptive feedback scheme of AQA-AODV and its route recovery mechanism allow getting an important decrease in 
packet loss (Figure 3.18) and delay (Figure 3.19) while it is makes more efficient use of the available bandwidth of the route. This fact provides better conditions to video streaming applications as demonstrated by the results obtained in the study that will be discussed in the next Chapter.

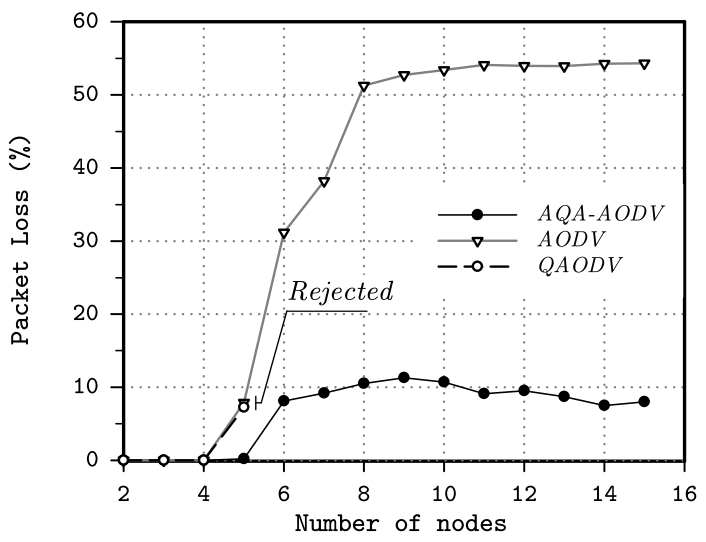

Figure 3.18. Packet Loss with variable chain length

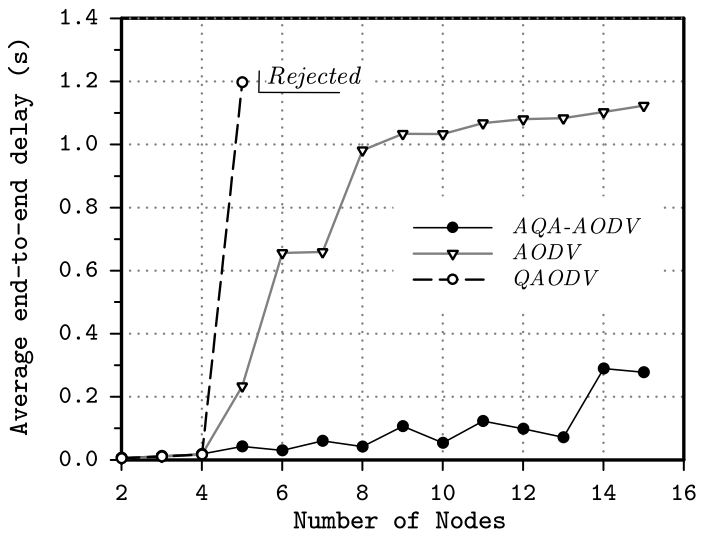

Figure 3.19. Average end-to-end delay with variable chain length

Figure 3.20 shows the number of link failures and the Connection Setup Latency (CSL). It is noteworthy that, when the chain has 5 or more nodes, the transmission rate (0.35Mbps) is not supported efficiently and the number of link failures drastically increases for AODV, which is about $25 \%$ higher than link failures for AQA-AODV. CSL in case of AQA-AODV is the lowest and varies from $0.08 \mathrm{~s}$ (for a chain of 5 nodes) to $0.95 \mathrm{~s}$ (for a chain of 15 nodes) because of reduced recovery time in comparison with AODV. Therefore, AQA-AODV could be more efficient in real-time data transmissions (e.g. video streaming).

Regarding QAODV, its admission control system only accepts the traffic flow of 0.35 Mbps when the number of hops between source and destination is less than 4 . In these cases, QAODV presents a similar behaviour to AODV in terms of throughput, delay and 
packet loss. However, its route recovery process is less efficient due to the delay incurred in identifying a link failure and the larger latency in establishing the routes. As in results of the previous scenario, the label "rejected" was added on the curves of QAODV in order to denote that the traffic flow is rejected by the admission control system when the route cannot support the requirements of the flow.

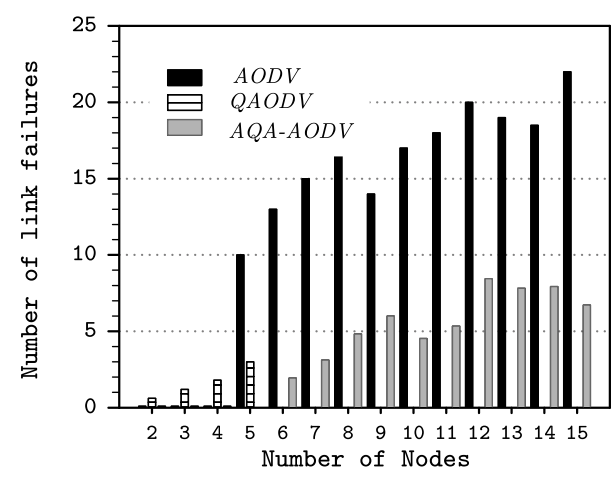

(a)

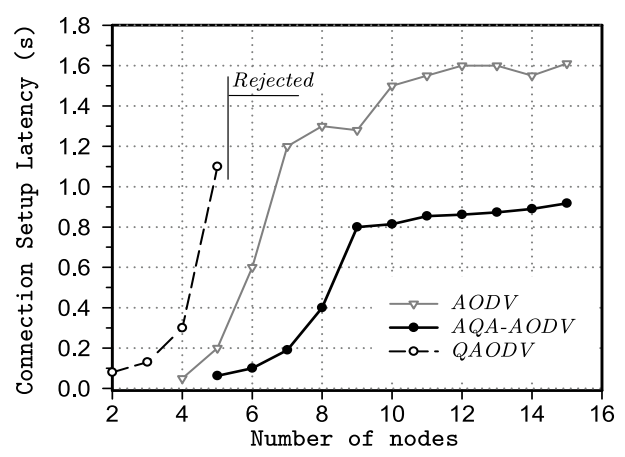

(b)

Figure 3.20. Number of link failures and Connection Setup Latency (CLS) vs. number of nodes

\subsubsection{Scenario 3: random mobile topology}

In the third scenario, 30 nodes move in a $1000 \mathrm{~m} \times 1000 \mathrm{~m}$ area according to the random waypoint model with pause time set to $5 \mathrm{sec}$. The nodes move toward a random destination using a speed between $0-2 \mathrm{~m} / \mathrm{s}$. A random source-destination pair sends video packets using a request rate between 0.1 and $1.0 \mathrm{Mbps}$. The traffic flow between source and destination consists of a CBR (Constant Bit Rate) flow over UDP. In addition to the main traffic flow, 5 CBR flows over of $10 \mathrm{Kbps}$ each are introduced randomly as background traffic in the network.

Figure 3.21, Figure 3.22 and Figure 3.23 show the results of the simulations in which the packet loss, average end to end delay and throughput are plotted versus the requested rate by source node.

In terms of packet loss (Figure 3.21), AQA-AODV shows an important improvement over AODV, which achieves very high packet losses for some requested rates. For example, the packet loss is between $1.3 \%$ and $10 \%$ using AODV, whereas using AQA-AODV the packet loss remains lower than $2 \%$. On the other hand, the results of Figure 3.22 shows that the average end to end delay of AQA-AODV is always below $70 \mathrm{~ms}$, whereas, the end to end delay of AODV increases badly when the transmission rate increases from 500 kbps to $1000 \mathrm{kbps}$. With AODV, the maximum average end to end delay reaches $300 \mathrm{~ms}$ at $1000 \mathrm{kbps}$, about 4 times higher than using AQA-AODV. Although QAODV shows a delay lower than AODV, only can support traffic flows below $0.5 \mathrm{Mbps}$ in this scenario. 


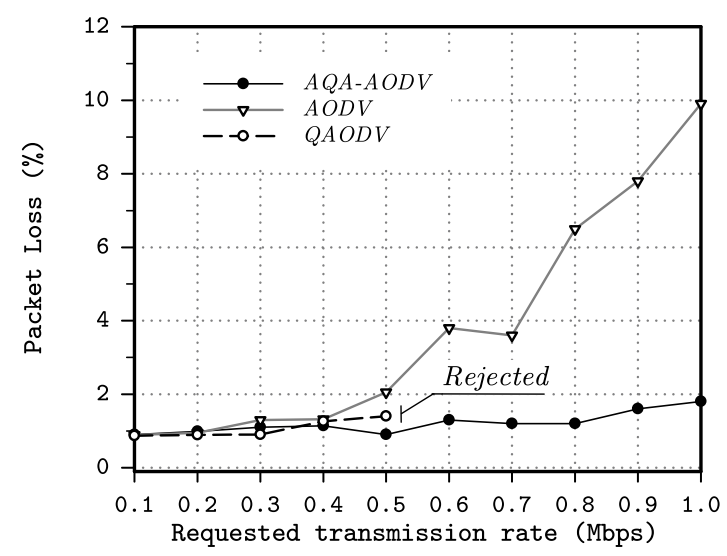

Figure 3.21. Packet Loss with variable requested rate.

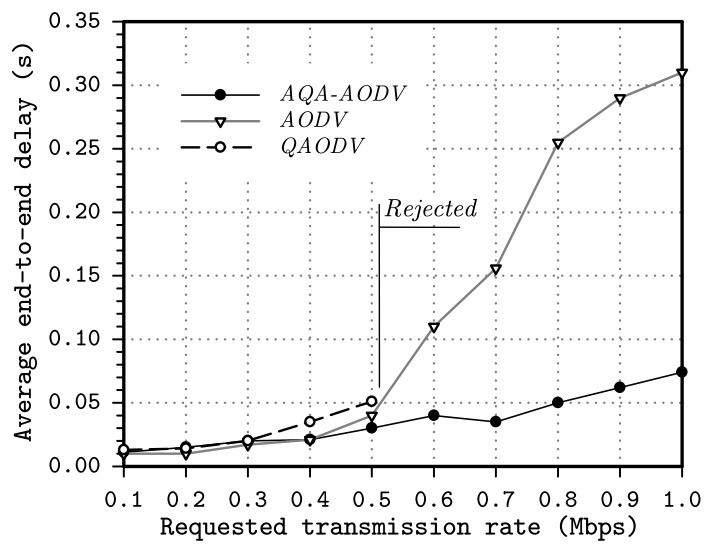

Figure 3.22. Average end-to-end delay with variable requested rate.

As seen in Figure 3.23 the total network throughput achieved with AQA-AODV is very close to throughput achieved using AODV. Although there is a degradation of the AQAAODV performance in scenarios with high mobility (compared with static scenarios) since the nodes need a higher time for exchanging information about the network status, the proposed protocol still shows better performance than the other alternatives.

An improvement of the route recovery process is also observed in the performance evaluation of AQA-AODV. As observed in Figure 3.24a, in the mobile scenario the frequencies of route break increase in the network compared with previous static scenario where the link failures were caused by congestion in the nodes. Each time a route breaks due to node mobility, there is some latency in new connection setup (which includes route break detection time, route discovery time and recovery time) and packet gets lost during connection setup period, which could explain the growth of the packet loss. Figure 3.24b shows that the CSL of AQA-AODV is always lower than CSL of AODV (about $50 \%$ of average lower than CSL for AODV). 


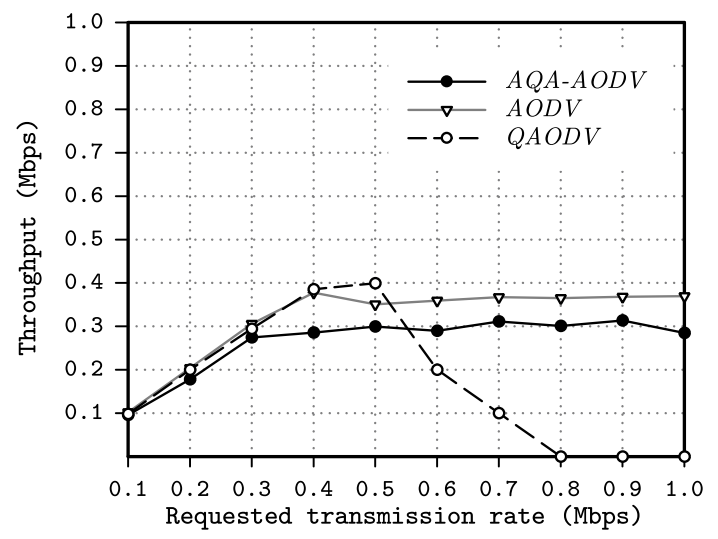

Figure 3.23. Throughput with variable requested rate

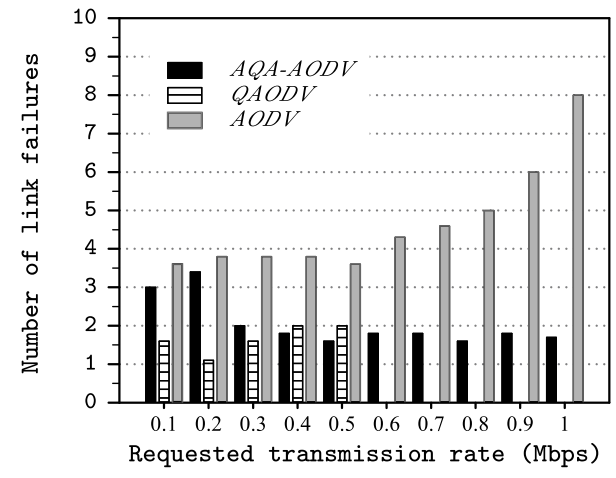

(a)

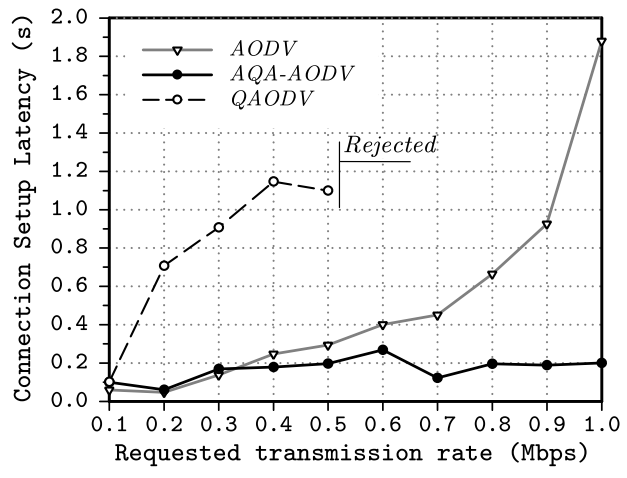

(b)

Figure 3.24. Evaluation results of the route recovery mechanism: (a) Number of link failures and (b) CSL (Connection Setup Latency) as a function of the requested rate.

\subsubsection{Conclusions}

The analysis of the performance evaluation of the proposed protocol shows that it provides significant improvements in all network scenarios considered. The feedback scheme of AQA-ADOV is presented as a suitable strategy for supporting rate-adaptive transmission where traffic source can adjust its data rate according to the network conditions. This feature provide an efficient method to avoid network congestion reducing the packet loss and the average packet delay. At same time, the achieved throughput is similar to that achieved with other reactive protocol as AODV. This demonstrates that, there is an increased efficiency in the use of the bandwidth since, although with AQA-AODV the source node sends packets to the network a lower rate, most of them reach their destination. In contrast, with AODV more packet are sent by source node, but a high percentage of them are lost along the route. 
The feedback scheme of AQA-AODV also presents significant advantages over the admission control system used by others QoS routing protocols as QAODV. Although the admission control scheme helps avoid network congestion rejecting flows that cannot be transmitted, this scheme has very low flexibility. This feature is not consistent with the requirements of the recent application development paradigms that promote the content adaptation to the network changes.

\subsection{Summary}

A novel QoS-aware routing protocol (AQA-AODV) is proposed in this chapter for carrying out time-sensitive communications over wireless ad hoc networks. AQA-AODV operates over a cross-layer feedback scheme which provide information to the application about the network status in order to avoid the transmission of data packets with a rate higher than the maximum rate that can be supported along the route. The key elements of the architecture of AQA-AODV were detailed in this chapter. In summary, three modules were analysed: bandwidth estimation module, routing module and the route recovery module. The first module includes the algorithms for local bandwidth estimation as well as the prediction of the consumed bandwidth taking into consideration the interference between packets of the same flow. Secondly, the incorporation of the bandwidth estimation in the route discovery process was described. Finally, the route recovery module was presented. This module incorporates into AQA-AODV new mechanisms for connection re-establishment to destination after a link failure, with the QoS conditions that had been negotiated during the initial route discovery phase.

The results of the performance evaluation show that the proposed protocol reduces significantly both the dropping rate and the end-to-end delay without impact the overall end-to-end throughput. Moreover, the results about the connection setup latency and the number of link failures shows that the proposed mechanisms for route recovery are perfectly integrated into the adaptive feedback scheme of AQA-AODV.

Later in next Chapter, the use of AQA-AODV in the provision of rate-adaptive video delivery services over MANETs, will be analysed. 


\section{Chapter 4 Adaptive Video Streaming over MANETs using AQA-AODV}

This chapter analyses the combined use of the cross-layer mechanisms included in AQA-AODV and the Scalable Video Coding (H.264/SVC). The main aim is to build a solution for supporting adaptive video streaming that can significantly contribute to increasing the quality of video streaming services while the bandwidth efficiency is achieved. In addition, an evaluation framework for adaptive scalable video streaming is also presented and evaluated. This framework has been developed in order to solve the lack of video evaluation tool-sets that support simulation tests that involve rate-adaptive video transmission using the H.264/SVC standard.

\subsection{Adaptive scalable video streaming}

As described in previous chapter, AQA-AODV provides mechanisms not only for route discovery and route maintenance but also for estimating the available bandwidth. Moreover, it also provides a cross-layer feedback for sending information about the network state to application layer. Nevertheless, in a realistic scenario are necessary additional techniques to carry out the content adaptation taking into account the network conditions. Some possibilities were analysed in the revision presented in Chapter 2. In summary, among the adaptive streaming techniques are: $i$ ) semantic techniques, $i$ i) having multiple versions for the same content and iii) scalable coding. Either option can be used to adapt the encoding rate by modifying characteristics of multimedia content, such as the resolution (dimensions of the video), the number of frames per second or the quality of the frames. Whereas the first option involves complex analysis of semantic information, the techniques of multiple versions require extra storage capacity since it will be necessary to store different copies of each video, with different quality levels, which is a non-scalable option. The third option allows us to have different levels of scalability in a single video stream. Therefore, it is not necessary to have multiple versions of the same content with different levels of quality, 
saving storage space. Thereby a wide range of terminals over heterogeneous networks can be served with a single version of the video. This is possible because the video stream will consist of several layers, each with different characteristics of quality. The number of layers that are sent to the client will depend on the state of network. This technique is called SVC (Scalable Video Coding) [39]. SVC has the advantage of scalability with a low computational cost, which is a very desirable feature especially when the service is accessed by a large number of users.

This Chapter presents an adaptive solution based on the combination of SVC with the available bandwidth estimation algorithms of AQA-AODV. This solution is able to adjust of the content quality to the transmission condition in order to avoid network congestion as well as further degradation of the quality of experience $(Q o E)$. This idea is illustrated in Figure 4.1. The scalable video coding (H.264/SVC) provides features to generate different representations of the same video integrated within a same bit stream. A video encoded using the $S V C$ standard has a layered structure where the layers correspond to different quality, spatial or temporal representations. A SVC video is composed of a base layer, which corresponds to the lowest representation, and one or more enhancement layers that increase the video quality when these are added to the base layer. The layered scheme of SVC can provide higher robustness during video streaming over networks with continuous fluctuations of the bandwidth. SVC allows the sender to adapt the bit rate of the video traffic adding or removing SVC layers from the video stream based on the estimation of the available bandwidth (as described the bottom part of Figure 4.1). Therefore, in order to adaptively control the bit rate of the video source, the adoption of cross-layer mechanisms in video streaming is required. To build a realistic solution, the cross-layer mechanism of AQA-AODV is applied. This mechanism involves information exchange and the generation of an adaptive video stream with adjusted bit rate as well as the establishment of optimal routing policies.

Before making the description of the integration of AQA-AODV and the SVC coding, it is important to review some concepts related to the SVC coding. Therefore, a brief introduction about SVC is presented in next section.

\subsubsection{Scalable Video Coding}

This section briefly describes the main technical features of $S V C$, the scalable extension of the H.264/AVC standard [39]. A more detailed explanation of the fundamentals of SVC can be found in the study of Schwarz et al. [86].

With H.264/SVC, the encoder produces a scalable bit-stream, which consists of a multiple layers. A base layer provides a basic video quality (e.g. low spatial or temporal resolution) and adding enhancement layers improves the quality (e.g. increases spatial resolution or frame rate). There are three modes of video scalability supported by SVC: temporal scalability, spatial scalability and quality scalability. When using temporal scalability, layers improve the frame rate. With spatial scalability, the base layer is coded at a low spatial resolution and enhancement layers give progressively higher spatial resolution. Finally, quality scalability refers to scaling in terms of the level of compression applied to the source video. This is primarily controlled using the quantization parameter $(Q P)$. With quality scalability the base layer contains a strongly 
compressed version of each picture, and enhancement layers incorporate more information to increase the SNR (Signal-Noise-Ratio) value.

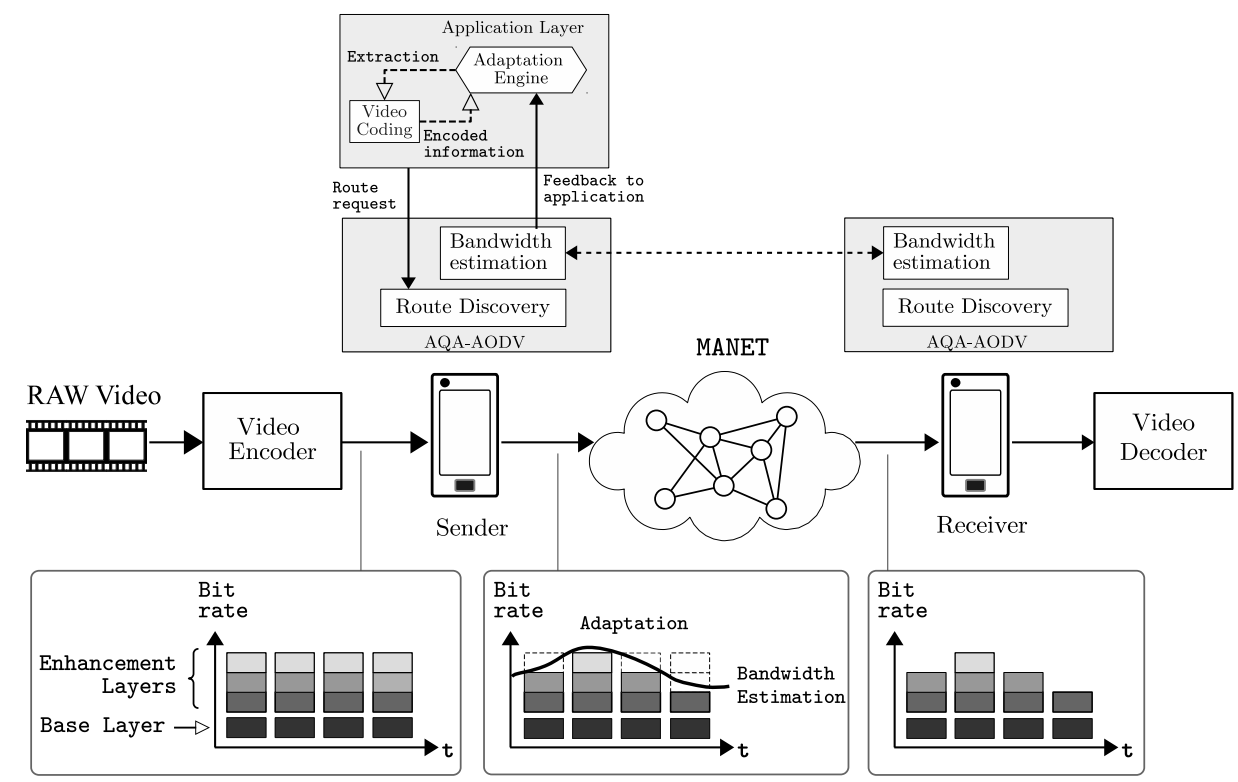

Figure 4.1. Adaptive scalable video streaming using AQA-AODV.

The H.264/SVC standard supports combined scalability, i.e. a scalable video can use any combination of the three types of scalabilities. For instance, Figure 4.2 shows a SVC stream encoded with four temporal levels $\left\{\mathrm{T}_{0}, \mathrm{~T}_{1}, \mathrm{~T}_{2}\right.$ and $\left.\mathrm{T}_{3}\right\}$ and two quality levels $\left\{\mathrm{Q}_{0}\right.$ and $\left.\mathrm{Q}_{1}\right\}$. Thus, eight scalable layers are generated by combining these levels as is listed in the Figure 4.2. The base layer consists of the lowest temporal resolution $\left(\mathrm{T}_{0}\right)$ and the lowest quality level $\mathrm{Q}_{0}$ (i.e. frames 1 and 8). In addition, an example of enhancement layer can be the layer consisting of the temporal layer $T_{2}$ and the quality level $Q_{1}$. This encoded video stream exploits the hierarchical prediction structure using B-pictures for enabling temporal scalability. Moreover, the coding structure of the quality scalability uses the key picture concept (other coding structures can be consulted in [86] and [87]).

In $H .264 / S V C$, the codec is divided in two subsystems: the Video Coding Layer $(V C L)$ and the Network Abstraction Layer $(N A L)$. Basically, the $V C L$ is in charge of the source video coding and the $N A L$ is the interface between the encoder and the actual network protocol, which will be used to transmit the encoded bit-stream. This thesis focuses on the NAL subsystem, since it provides the required information to identify the data relating to each layer.

In the VCL subsystem, each picture of the video is partitioned into macroblocks that each covers a rectangular area of $16 \times 16$ luminance samples. The macroblocks of a picture are organized in slices each of which can be parsed independently of other slices in a picture. Each slice encoded in $V C L$ is encapsulated into Network Abstraction Layer Units $(N A L U)$, which are suitable for transmission over networks. 


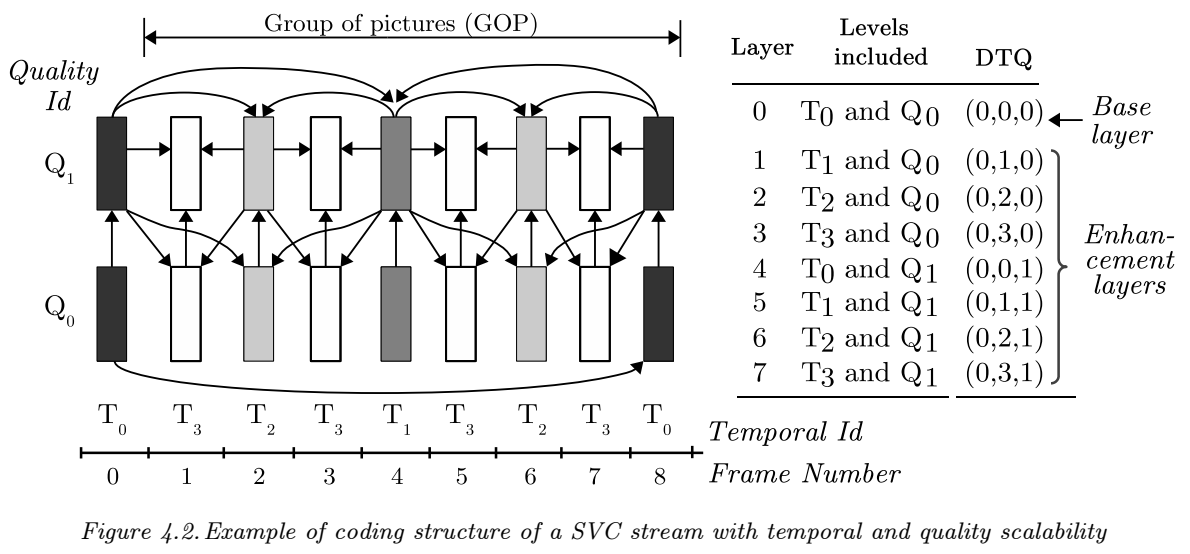

The main characteristic of the NALUs used in SVC is a sequence of 3 bytes that contain three identifiers: dependency identifier $(D I D)$, temporal identifier $(T I D)$ and quality identifier $(Q I D)$. These identifiers represent a point in the spatial, temporal and quality scalable dimensions, respectively. The values of $D I D, T I D$ and $Q I D$ are also known as $D T Q$ parameters. For instance, a $N A L U$ of the base layer should be identified as $(0,0,0)$ and for instance the enhancement layer consisting of the $T_{2}$ and $Q_{1}$ levels should be identified as $(0,2,1)$. The inspection of the DTQ values permits to identify the NALUs belonging to a specific enhancement layer. This fact is particularly important since the $N A L U$ s may be identified and removed from the $S V C$ encoded video, in order to reduce the bit rate. The remaining NALUs may integrate a valid video bit-stream with a lower visual quality. Nevertheless, the dependencies of the layers would be taken into account. Layers in $S V C$ can be decoded independently but there is a logical dependency between them. This interdependency must be considered in order to obtain a correct decoding of the video. In the example shown in Figure 4.2, the arrow lines represent dependencies between frames in a combined scalable stream. For instance, the frame 2 of the layer $\mathrm{T}_{2} \mathrm{Q}_{1}$ depends on the layer $\mathrm{T}_{0} \mathrm{Q}_{1}$ and the layer $\mathrm{T}_{1} \mathrm{Q}_{1}$ (which also depends on base layer). Because of these dependencies, discarding a quality layer from a reference frame (e.g. frame 2) affects the quality of dependent frames (e.g. frames 1 and 3).

\subsection{Video transmission evaluation}

Video transmission evaluation requires some characterization of the video. Basically, three methods can be used to characterize encoded video in networking research: $i$ ) video bit stream, ii) video traffic model, and iii) video traffic trace. The first option involves the generation of a video bit stream using the output of a video encoder, so that it contains the complete video information. This method is focused mainly on real networks. Therefore, expertise and equipment are necessary for both video coding and networking in order to perform research studies on video transmission evaluation with actual bit streams. In addition, the difficulty to carry out a reliable assessment of new protocols is another limitation. Some examples of video bit stream include REALTRACER [88] and ORB [89]. The second method is based on using video traffic models, which are mathematical models that describe the essential properties of the real 
video streams. These models are generally developed based on the statistical analysis of video trace samples of real video traffic. Several models for video traffic have been proposed in the literature. For instance, the studies of Dai et al. [90], Ivrlac et al. [91] and Espina et al. [92] describe traffic models for H.264/AVC encoded video and the recent surveys presented by Tanwir and Perros [93] [94] where several VBR video traffic models are analysed. Regarding traffic models for scalable video, can be consulted in the references [95], [96] and [97]. Although using mathematical modelling has advantages such as the rapid generation of the results for video traffic and queue statistics analysis, the complexity of the actual heterogeneous networks makes realistic simulation a better tool, especially when an evaluation of the perceived visual quality of the video is required. Finally, the third method is the generation of video traffic traces, which are an abstraction of real video stream. A video trace is a text file where the characteristics of real video traffic, such as the frame number, frame type and frame size, are described. These video traces can be employed to simulate video traffic in a wide range of network simulation tool, which has been considered a viable approach for evaluation of various video delivery systems. Several simulation tools of video traffic based on trace files have been developed, for example the EvalVid tools-set [98] and its extended versions for NS2 suggested by Ke et al. [44] and Lie et al. [99]. These platforms support trace-driven simulations of H.263 and H.264/AVC video transmission. One possibility for support simulation with rate-adaptive video transmissions is to use the Evalvid-RA (Evalvid Rate Adaptive) framework developed by Arne Lie et. al. [99]. Evalvid-RA is a simulation tool that supports adaptive video transmission using MPEG-4 VBR (Variable Bit Rate) videos. The adaptive mechanism of this platform involves switching between different preencoded versions of the video (each with different levels of quality). However, a more efficient solution is provided by the H.264/SVC standard, which supports encoding of a video in different qualities within the same bit-stream. This includes different resolutions, different frame rates (fps) and different quality levels, avoiding the compression of a video into multiple representations at different bitrates, as analysed in Chapter 2.

Concerning simulation platforms for scalable video, in recent years, have been implemented few solutions, such as SVEF (Scalable Video-streaming Evaluation Framework) [100]. SVEF is a tools-set for empirical evaluation of real- time SVC streaming. However, its practical application is limited because SVEF tool-set cannot support experiments that involve new technologies or network protocols. Moreover, it does not take the spatial scalability into consideration and only supports SVC videos with a single dependency layer. Other evaluation platforms for scalable video streaming are EvalSVC (Evaluation of SVC) and myEvalSVC simulation framework, which were developed by Le et al. [101] and Ke [102] respectively. These simulations platforms extend the EvalVid framework in order to provide a simulation framework where SVC video transmissions can be evaluated over a general network simulator as NS-2. Nevertheless, these frameworks were not designed to allow source traffic to adapt its bit rate adding or removing layers from the video stream. Therefore, they do not provide a suitable solution for rate-adaptive video investigation. Consequently, an open issue in the development of adaptive video delivery systems is the lack of freely available video 
evaluation tool-set where researchers and network developers can test the performance of their network-adaptive techniques. In order to solve the above-mentioned problem, it is proposed the SVCEval-RA evaluation framework for more realistic simulations of adaptive scalable video transmission.

\subsection{Proposed evaluation framework for scalable video streaming}

This section describes a novel simulation framework called SVCEval-RA (SVC Evaluation Platform for Rate-Adaptive Video), which can be used in the assessment of rate-adaptive video streaming using Scalable Video Coding. This approach is a modified and enhanced version of the myEvalSVC tool-set. More precisely, a new procedure have been implemented in SVCEVal-RA in order to adapt the bit rate of the traffic source adding or removing SVC layers from the video stream based on the estimation of the available bandwidth. This procedure (referred to hereafter as the Adaptive SVC scheme of SVCEval-RA) provides better conditions to carry out performance evaluations of network protocols for adaptive video transmission, compared with the other evaluation platforms. For providing a more realistic simulation environment, the developed algorithms have been integrated in the simulation tool NS-2. Nevertheless, as discussed later, the proposed architecture can be integrated within any network simulator.

The main contributions of SVCEval-RA compared to referenced works are:

1. The SVCEval-RA framework is enhanced to support rate adaptive scalable video.

2. The modular structure of SVCEval-RA makes possible that third party modules can be easily integrated in order to extend its capabilities. For instance, transport protocols for video streaming with their own algorithms for network resource estimation, such as TFRC (TCP Friendly Rate Control) and RTMP (Real Time Messaging Protocol).

3. Simulation studies using adaptive SVC techniques can be performed using SVCEval in different network scenarios (e.g. Internet, WLANs and MANETs) where video streaming services require adaptation to the network conditions, such as DASH.

4. In contrast to the other simulation platforms, SVCEval-RA supports SVCencoded video flows with both quality and temporal scalability.

5. A simulator-independent tool, written in $\mathrm{C}++$, is also provided. This software integrates, in only one application, the tasks that must be executed for the interfacing between the encoder and the simulator.

In next sub-sections, it is described the main characteristics of the proposed SVCEvalRA framework and a performance evaluation of SVCEval-RA will be discussed in Section 4.4. Subsequently, Section 4.5 will focus on showing how the mechanisms for estimating the available bandwidth provided by AQA-AODV are integrated in the SVCEval-RA platform in order to support simulations that involve the transmission of scalable video in mobile ad hoc networks.

The SVCEval-RA framework performs video transmissions over a packet network using a general network simulator. Thus, any specific trace format defined by the integrated 
simulator can be easily adapted in the proposed evaluation platform. However, SVCEval-RA has been integrated to the NS-2 simulator.

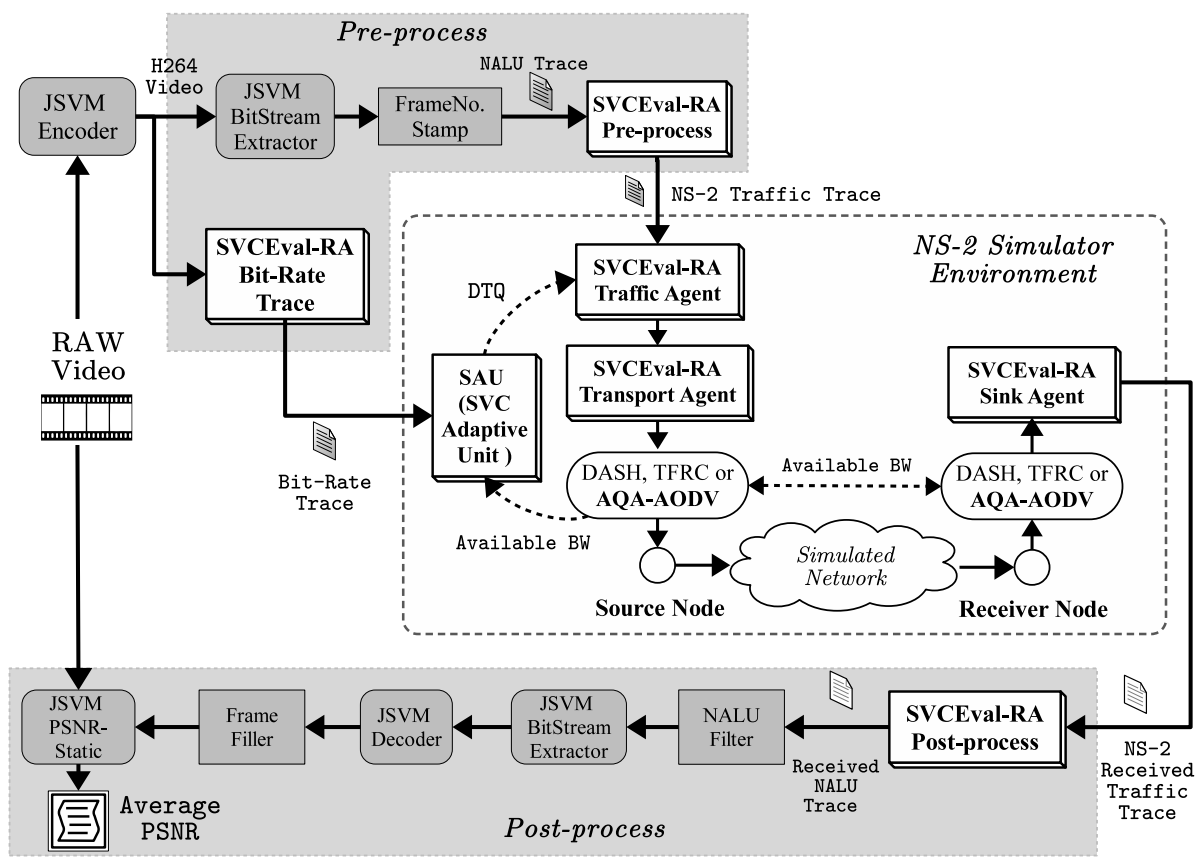

Figure 4.3. Workflow of the SVCEval-RA framework

Figure 4.3 illustrates the workflow of the SVCEval-RA evaluation framework. The software modules inherited from the JSVM (Joint Scalable Video Model) package [103] and SVEF (Scalable Video coding Streaming Evaluation Framework) scripts [100] are represented in grey. The whole process, from the encoding of the original video source to the evaluation after the streaming over a simulated network can be summarized in four phases, better detailed in the following sub-sections: encoding, pre-processing, simulation and post-processing. In the encoding phase, the original uncompressed video is encoded in H.264/SVC format through the JSVM Encoder and a corresponding trace file (NALU trace) is produced. Pre-process consists of the generation of the Bit Rate Trace and the NS-2 Traffic Trace file. During simulation, the NALUs are forwarded to the receiver through a simulated network. Moreover, the source can adjust its data rate by selecting a set of $S V C$ layers to be transmitted based on the estimation of the available bandwidth. After simulation, the received NALU trace is processed in order to produce a $Y U V$ file, which may be affected by missing frames due to transmission losses, unsatisfied decoding dependencies or excessive delay. Finally, the PSNR (Peak Signalto-Noise Ratio) is evaluated by comparing the original uncompressed video with the distorted one. In Figure 4.3, the different phases are further detailed. 


\subsubsection{Video Encoding}

The framework uses RAW video files stored in the standard $Y U V$ format. The RAW video is then encoded according to the H.264/SVC coding scheme. Encoding is performed using the JSVM software, which is the reference implementation of the H.264/SVC standard. In particular, the JSVM tool H264EncoderStatic encodes a YUV video by configuring all the H.264/SVC encoding settings, including the type of scalability to be used, and the number of layers that will compose the output stream. After that, the NALU trace file is produced from the H.264/SVC encoded video using the BitStreamExtractor JSVM tool. This NALU trace file includes a different register for each packet of the $N A L U$ sequence. Among other parameters, each register specifies the size of the packet, the $D T Q$ values and the packet type, which are needed during the decoding phase.

\subsubsection{The Pre-process}

The first task of the pre-process is to add the frame number information to the NALU trace file using the F-N Stamp tool. On the other hand, the Bit Rate Trace is generated. This Bit Rate Trace file provides information about the bit rate required to transmit each layer of the SVC encoded video. Table 4.1 shows a fragment of a Bit Rate Trace. The meanings of all fields are as follows: layer id, frame rate $(\mathrm{Hz})$, bit rate, $D I D, T I D, Q I D$ and $P S N R(\mathrm{~dB})$. For instance, according to the Table 4.1 , layer 2 with $D T Q$ values $(0,3,0)$ has a bit rate of $273.3 \mathrm{kbps}$ which is the required bit rate to transmit the layer 2 and its dependent lower layers. The value of $27.1 \mathrm{~dB}$ is an estimation of the PSNR value that would be obtained if all NALUs of the layer 2 and the dependent layers were correctly received after the video transmission.

Table 4.1. Example of Bit Rate Trace

\begin{tabular}{|ccccccc|}
\hline $\begin{array}{c}\text { Layer } \\
\text { id }\end{array}$ & $\begin{array}{c}\text { Frame } \\
\text { rate }\end{array}$ & Bit-rate & DID & TID & QID & PSNR (dB) \\
0 & 3.7500 & 139.90 & 0 & 1 & 0 & 19.6600 \\
1 & 7.5000 & 197.40 & 0 & 2 & 0 & 22.4023 \\
2 & 15.000 & 273.30 & 0 & 3 & 0 & 27.1010 \\
3 & 30.000 & 335.40 & 0 & 4 & 0 & 35.5127 \\
$\ldots$ & $\ldots$ & $\ldots$ & $\ldots$ & $\ldots$ & $\ldots$ & $\ldots$ \\
\hline
\end{tabular}

The diagram shown in the Figure 4.4 describes in general form, the main tasks involved in the generation of the Bit Rate Trace file. Basically, the procedure shown in Figure 4.4 involves generating sub-streams extracting each SVC layer from the entire scalable video sequence. These extracted sub-streams are decoded and then the PSNR is calculated. In the measurement of the PSNR both sequences (sub-stream and original $R A W$ video) must have the same spatial and temporal resolutions, therefore frame copy operations (Frame Filler) must be applied to the extracted sub-streams. This method is suggested by Sohn et al. in [104] and it consists of duplicating the frames of the lower layer so that both sequences have the same number of frames. Section 4.5.1 provides a more detailed description of the method used in this study to calculate the PSNR of the test sequences. As described Figure 4.3, after the Bit Rate Trace file is generated, the SVCEval-RA Preprocess tool adapts the NALU trace file to the appropriated format required by the SVCEval-RA Traffic Agent. The new trace file, named NS-2 Traffic Trace, contains the following fields for each record: sending time, frame size, the DTQ values (DID, TID, 
QID) and the number of fragmented packet. Finally, the Bit Rate Trace and the NS-2 Traffic Trace are sent to the SVC Adaptive Unit and the SVCEval-RA Traffic Agent integrated in NS-2, respectively.

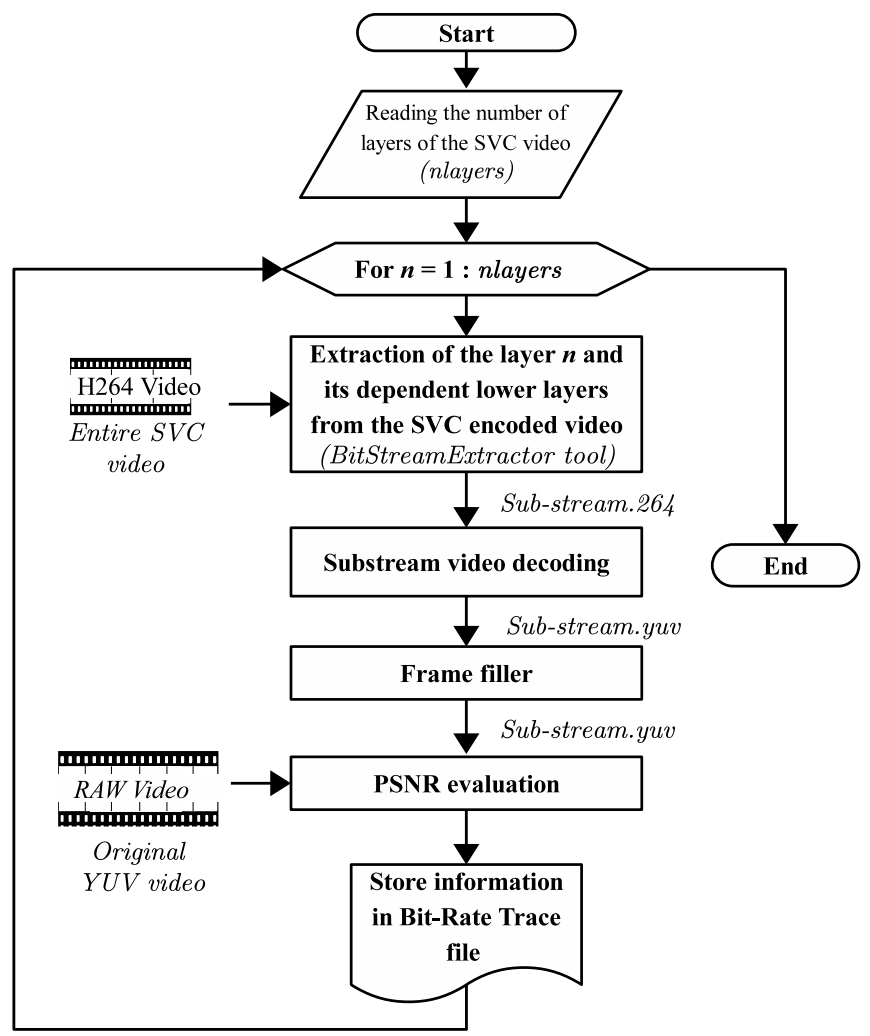

Figure 4.4. Flowchart of the generation of the Bit Rate Trace

\subsubsection{Simulation}

As shown in Figure 4.3, four NS-2 agents were implemented. In the sender side are the SVCEval-RA Traffic Agent, the SVCEval-RA Transport Agent and the SVC Adaptive Unit (SAU). In the receiver side is the SVCEval-RA Sink Agent. The SVCEval-RA Traffic Agent reads each record from the NS-2 Traffic Trace file, generates the corresponding number of packets and sends them to the lower layer at the appropriate time according to the user settings specified in the simulation script file. The tasks related with the adaptive SVC scheme are executed in the SAU and the Traffic Agent, which makes possible the successful adaptation of the sending rate transmitting only the SVC layers that can be efficiently handled by the network.

The SVCEval-RA Transport Agent is an extension of the UDP agent of NS-2. More specifically, it records the sending time, packet ID and the packet size of each 
transmitted packet. Then those packets go down to the lower layers and to the simulated networks.

As mentioned above, the proposed framework can be integrated with different protocols (e.g. TFRC or AQA-AODV), which must provide an algorithm to estimate the available bandwidth along a route. The value of available bandwidth estimated by the streaming protocol is sent to the SVCAdaptive Unit (SAU). The SAU searches in the Bit Rate Trace the DTQ parameters of the highest SVC layer that can be transmitted without exceeding the bandwidth constraint. The SVC layer selected is the layer with a bit rate value less or equal than the data rate supported by the network.

The SAU sends the DTQ parameters of the selected layer to the SVCEval-RA Traffic Agent. It will transmit only the NALUs identified with the same DTQ parameters as well as the NALUs of the dependent lower layers.

At the receiving side, the SVCEval-RA Sink Agent is used to receive the fragmented video packets sent by the SVCEval-RA Transport Agent. This agent also records the receiving time, frame number, packet size, DID, TID, QID, packet ID and the sending time in the Receiver Traffic Trace file. The diagram shown in the Figure 4.5 describes in general form, the main tasks involved in the adaptation process of video transmission using the Adaptive SVC scheme.

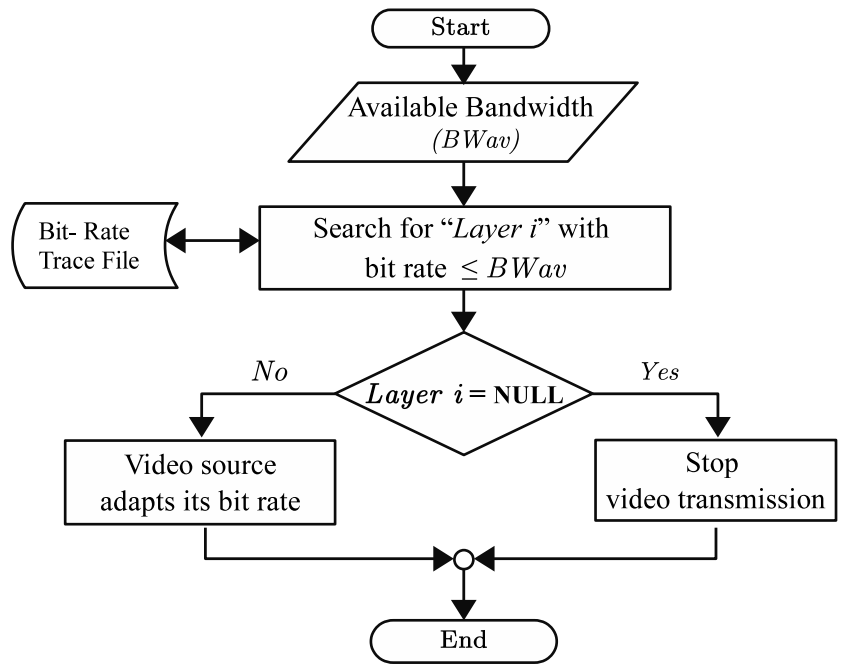

Figure 4.5. General adaptation scheme of SVCEval-RA

\subsubsection{Post-processing and decoding}

The main post-processing tasks are illustrated in the lower part of Figure 4.3. After simulation, the Received Traffic Trace is first processed by the SVCEval-RA Post-process program in order to obtain the Received NALU Trace file. Afterward, the Received NALU Trace file is passed to the NALU-Filter tool which: $i$ ) removes NALUs received after the 
play-out buffer deadline, $i$ i) reorders the $N A L U$ s according to the sending order and, $i i i)$ removes NALUs with unfulfilled decoding dependencies.

The filtered Received NALU Trace file is used to reconstruct the H.264/SVC stream using the BitStreamExtractor and the JSVM Encoder tools. The obtained SVC distorted video can then be decoded to a YUV RAW file by means of the JSVM H264DecoderStatic tool. However, the resulting YUV video could have, in general, fewer frames than the original YUV video for two reasons: $i$ ) missing NALUs, and ii) static decoder skips lost frames without considering a replacement for them. Therefore, in order to properly evaluate the PSNR, the receiver YUV video is further modified by the Frame-Filler. This tool adds, for each missing frame, a copy of the immediately previous one obtaining the final YUV video. This final YUV video can be compared with the original YUV video in order to measure the PSNR for evaluating the end-to-end delivered video quality. Furthermore, others performance metrics such as the packet loss rate and end-to-end delay, can be obtained from the NS-2 Traffic traces generated during network simulation.

This section provides a performance analysis of SVCEval-RA. The main objective of the simulation study is to demonstrate the effectiveness of SVCEval-RA in the transmission of Adaptive SVC video over different network scenarios. Two video streaming strategies were evaluated in order to demonstrate the usefulness of the proposed framework. On one hand, a video sequence was transmitted according with the Adaptive SVC scheme of the SVCEval-RA platform. On the other hand, using the Non-Adaptive SVC scheme of the myEvalSVC framework, the same video sequence was also transmitted over the simulated scenarios. The two SVC streams were evaluated in terms of throughput of the video flow, the lost packet rate and the PSNR.

\subsection{Performance analysis of the SVCEval-RA framework}

This section provides a performance evaluation of SVCEval-RA. The main objective of our simulation study is to demonstrate the effectiveness of SVCEval-RA in the transmission of Adaptive SVC video over different network scenarios. Two video streaming strategies were evaluated in order to demonstrate the usefulness of the proposed framework. On one hand, a video sequence was transmitted according with the Adaptive SVC scheme of the SVCEval-RA platform. On the other hand, using the Non-Adaptive SVC scheme of the myEvalSVC framework, the same video sequence was also transmitted over the simulated scenarios. The two SVC streams were evaluated in terms of throughput of the video flow, the lost packet rate and the PSNR.

\subsubsection{Simulation environment}

The network simulator NS-2 has been used to test the performance of the proposed framework. The network scenarios considered in the evaluation are depicted in Figure 4.6. These scenarios provide persistent fluctuations of the available bandwidth in order to assess the rate adaptive mechanisms of SVCEval-RA during a streaming of a video sequence. 
The first network scenario (described in Figure 4.6a) has two parts: from $\mathrm{t}=10 \mathrm{~s}$ to $50 \mathrm{~s}$, the available bandwidth is decreased at intervals of 10 seconds from $1.5 \mathrm{Mbps}$ to 100 Kbps. In the second part, the available bandwidth is increased from $100 \mathrm{Kbps}$ to 1.5 Mbps at intervals of ten seconds. This network scenario evaluates the behaviour of the video source to abrupt fluctuations in the available bandwidth. Such variations are common in Internet data communication for instance, when the cross traffic in the bottleneck of the path varies significantly on account of arriving or departing traffic from other users [105].

The second scenario (represented in Figure 4.6b) supplies a constant low available bandwidth of $0.1 \mathrm{Mbps}$ with high bandwidth spikes of $1.5 \mathrm{Mbps}$. The duration of these spikes is increased over time from $1.5 \mathrm{~s}$ to $5 \mathrm{~s}$. This scenario aims to measure the reaction time of the video source upon extreme variations of the available bandwidth.

The third network scenario presents the opposite situation of the second scenario. Scenario 3 supplies a continuous high available bandwidth of $1.5 \mathrm{Mbps}$ with low bandwidth troughs of $100 \mathrm{Kbps}$ as shown in Figure 4.6c. Duration of these troughs is also increased over time, the first trough lasts $1.5 \mathrm{~s}$ increasing up to $5 \mathrm{~s}$ in the last one.

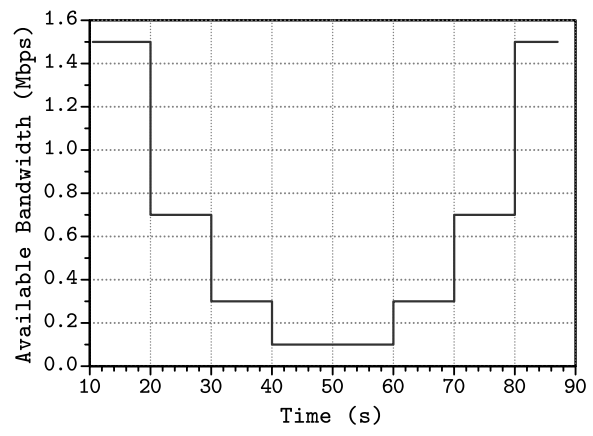

(a)

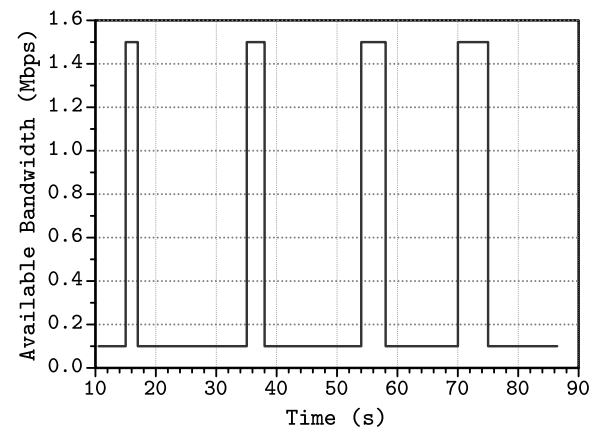

(b)

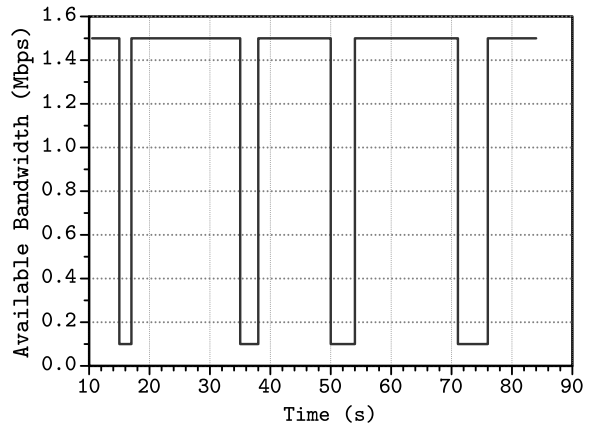

(c)

Figure 4.6. Network scenarios: Scenario 1: Persistent variations of the available bandwidth (a), Scenario 2: "Positive" peaks in the available bandwidth (b) and Scenario 3: "Negative" peaks in the available bandwidth (c)

The available bandwidth variations of the scenarios 2 and 3 are commons in practice, especially in 802.11 wireless networks [105]. These scenarios are intended to validate the 
performance of SVCEval-RA and the behaviour of the adaptive SVC scheme implemented in the SVCEval-RA Agents with more persistent and short-term bandwidth fluctuations compared with the scenario 1 .

Table 4.2. Video Parameters

\begin{tabular}{l|l}
\hline \multicolumn{1}{c|}{ Parameters } & \multicolumn{1}{c}{ Description/Value } \\
\hline Original Video file & YUV format \\
Size & 2300 frames \\
Frame per second & 30 \\
Encoded video file & H.264/SVC \\
Type of scalability & SNR (MGS) \\
GOP Size & 16 frames \\
\hline
\end{tabular}

As far as video source is concerned, a testing video of 2300 frames was generated concatenating the well-known video sequences "highway" and "hall" [106] with CIF resolution. The video sequence has been encoded according to H.264/SVC standard with two types of scalability: temporal and quality. As SVC codec, the JSVM codec [103] was used. All the values for the video related parameters are reported in Table 4.2.

Table 4.3. Description of the video layers of the coded sequence

\begin{tabular}{|c|c|c|c|c|}
\hline $\begin{array}{c}\text { Layer } \\
\text { ID }\end{array}$ & Ti Qi & $\begin{array}{c}\text { Frame } \\
\text { rate }\end{array}$ & $\begin{array}{c}\text { Aggregated } \\
\text { Bit rate } \\
\text { (Kbps) }\end{array}$ & $\begin{array}{c}\text { PSNR } \\
\text { (dB) }\end{array}$ \\
\hline 0 & $\mathrm{~T}_{0} \mathrm{Q}_{0}$ & 1.875 & 60.1 & 22.5 \\
\hline 1 & $\mathrm{~T}_{1} \mathrm{Q}_{0}$ & 3.75 & 80.5 & 24.7 \\
\hline 2 & $\mathrm{~T}_{2} \mathrm{Q}_{0}$ & 7.5 & 105.6 & 27.8 \\
\hline 3 & $\mathrm{~T}_{3} \mathrm{Q}_{0}$ & 15 & 136.3 & 31.3 \\
\hline 4 & $\mathrm{~T}_{4} \mathrm{Q}_{0}$ & 30 & 170.0 & 37.5 \\
\hline 5 & $\mathrm{~T}_{0} \mathrm{Q}_{1}$ & 1.875 & 94.9 & 22.9 \\
\hline 6 & $\mathrm{~T}_{0} \mathrm{Q}_{2}$ & 1.875 & 224.4 & 23.6 \\
\hline 7 & $\mathrm{~T}_{0} \mathrm{Q}_{3}$ & 1.875 & 365.3 & 24.1 \\
\hline 8 & $\mathrm{~T}_{1} \mathrm{Q}_{1}$ & 3.75 & 130.7 & 25.1 \\
\hline 9 & $\mathrm{~T}_{1} \mathrm{Q}_{2}$ & 3.75 & 307.4 & 26.3 \\
\hline 10 & $\mathrm{~T}_{1} \mathrm{Q}_{3}$ & 3.75 & 485.2 & 27.0 \\
\hline 11 & $\mathrm{~T}_{2} \mathrm{Q}_{1}$ & 7.5 & 182.3 & 28.4 \\
\hline 12 & $\mathrm{~T}_{2} \mathrm{Q}_{2}$ & 7.5 & 430.4 & 29.4 \\
\hline 13 & $\mathrm{~T}_{2} \mathrm{Q}_{3}$ & 7.5 & 660.9 & 30.6 \\
\hline 14 & $\mathrm{~T}_{3} \mathrm{Q}_{1}$ & 15 & 252.9 & 31.8 \\
\hline 15 & $\mathrm{~T}_{3} \mathrm{Q}_{2}$ & 15 & 639.1 & 32.7 \\
\hline 16 & $\mathrm{~T}_{3} \mathrm{Q}_{3}$ & 15 & 958.1 & 34.0 \\
\hline 17 & $\mathrm{~T}_{4} \mathrm{Q}_{1}$ & 30 & 331.1 & 38.0 \\
\hline 18 & $\mathrm{~T}_{4} \mathrm{Q}_{2}$ & 30 & 836.5 & 39.4 \\
\hline 19 & $\mathrm{~T}_{4} \mathrm{Q}_{3}$ & 30 & 1231.4 & 41.0 \\
\hline
\end{tabular}


The video sequence was encoded in five temporal layers (from $\mathrm{T}_{0}$ to $\mathrm{T}_{4}$ ). At the same time, three extra levels of quality scalability (from $\mathrm{Q}_{0}$ to $\mathrm{Q}_{3}$ ) at each temporal level were added. MGS (Medium Grain Quality Scalability) was used for quality scalability. The use of MGS layers for quality scalability allows source video to discard the data units from the enhancement layers without affecting the result bit-stream. In total, 20 video layers (from $\mathrm{L}_{0}$ to $\mathrm{L}_{19}$ ) were obtained from the combination of layers $\left(T_{i} Q_{\mathrm{j}}\right)$. Moreover, depending of the number of layers transmitted, the output bit rate varies from $60.1 \mathrm{kbps}$ (sending layer 0 alone) to $1.23 \mathrm{Mbps}$ (sending layers 0 to 19). The bit rates associated to each layer are shown in Table 4.3. These values are aggregated, which means that to transmit Layer 3 also the dependent lower layers (i.e. 0, 1, and 2) must be transmitted. Therefore, the total bandwidth required would be of $136.3 \mathrm{Kbps}$.

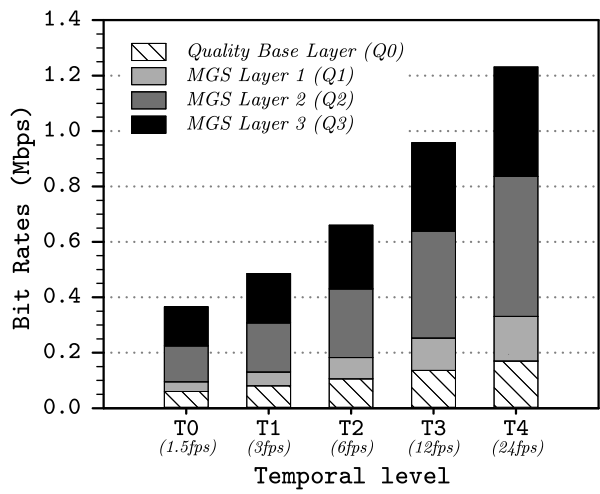

(a)

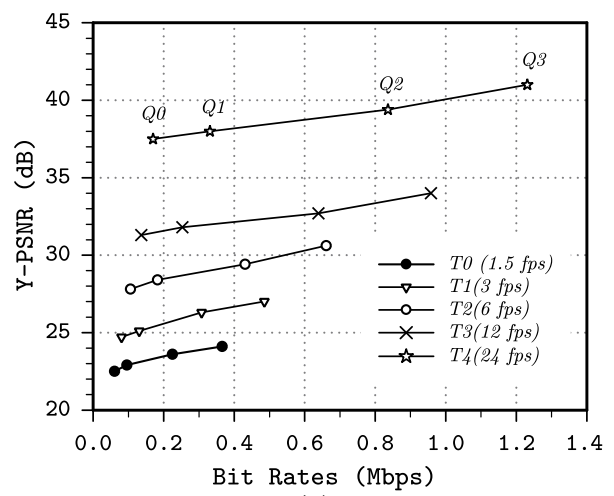

(b)

Figure 4.7. Description of the video layers (a) and Video properties used in the simulation study (b)

Figure 4.7a gives a graphical description of the bit rates obtained according to the temporal levels and the MGS layers. Moreover, a rate-distortion analysis in terms of average Y-PSNR (PSNR for the luminance component in the $Y U V$ colour space) versus average bit rate was computed off-line (see Figure $4.7 \mathrm{~b}$ ). The computation of the Y-PSNR curves were performed by stripping out the layers, measuring the average bit rate, decoding the resulting video, and computing the average Y-PSNR. Each of these curves represents a temporal layer and each point corresponds to a MGS layer (from $\mathrm{Q}_{0}$ to $\mathrm{Q}_{3}$ ). This figure describes the increase in the video quality ( in terms of Y-PSNR) depending on the number of quality and temporal layers that make up the video.

\subsubsection{Results}

This section describes the experimental results obtained in the simulation experiments. Each point in the curves represents the average over 10 simulations and a confidence level of $95 \%$. As mentioned above, two video streaming strategies were used: Adaptive $S V C$ and Non-adaptive SVC, in order to demonstrate the usefulness of the proposed framework. 


\subsection{2.a. Scenario 1: Persistent variations of the available bandwidth}

The results obtained for the scenario 1 are shown in the Figure 4.8. In addition, the available bandwidth fluctuation provided by the scenario 1 is also plotted. Figure 4.8a shows the throughput achieved during the video transmission. The lost packet rate and the luminance PSNR (Y-PSNR) are shown in the Figure $4.8 \mathrm{~b}$ and Figure $4.8 \mathrm{c}$ respectively. On the other hand, Table 4.4 summarizes mean values of the evaluated metrics for each segment in which a bandwidth variation was performed. In addition, the layers that were transmitted for each segment are also described in the column two, where BL means Base Layer and the combination of the T and Q parameters identifies the temporal and MGS layer, respectively.

As seen in Figure 4.8a, during the intervals from $10 \mathrm{~s}$ to $20 \mathrm{~s}$ and from $80 \mathrm{~s}$ to $86 \mathrm{~s}$ all SVC layers of the video sequence can be transmitted. This fact is explained because the rate required to send the highest layer (1.23 Mbps for layer 19, see Table 4.3) and its dependent lower layers can be supported by the link capacity. Consequently, all packets can be successfully transmitted (lost packet rate equal to 0 , see Figure $4.8 \mathrm{~b}$ ) and therefore the quality of the received video is the maximum that can be achieved (Figure $4.8 \mathrm{c})$.

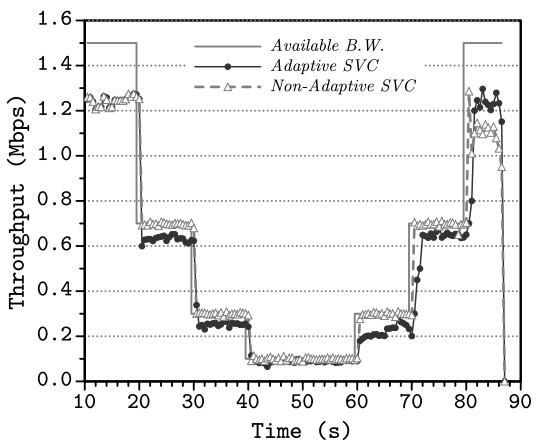

(a)

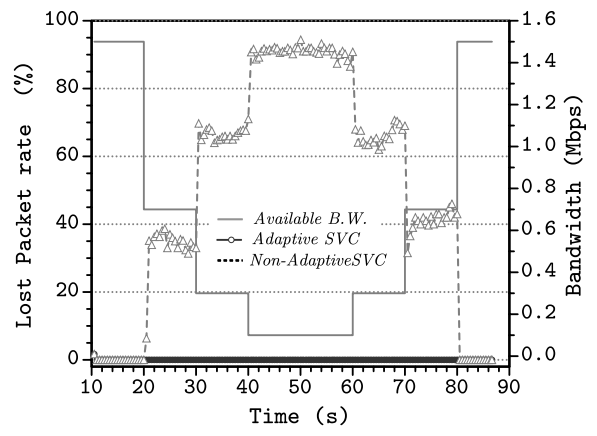

(b)

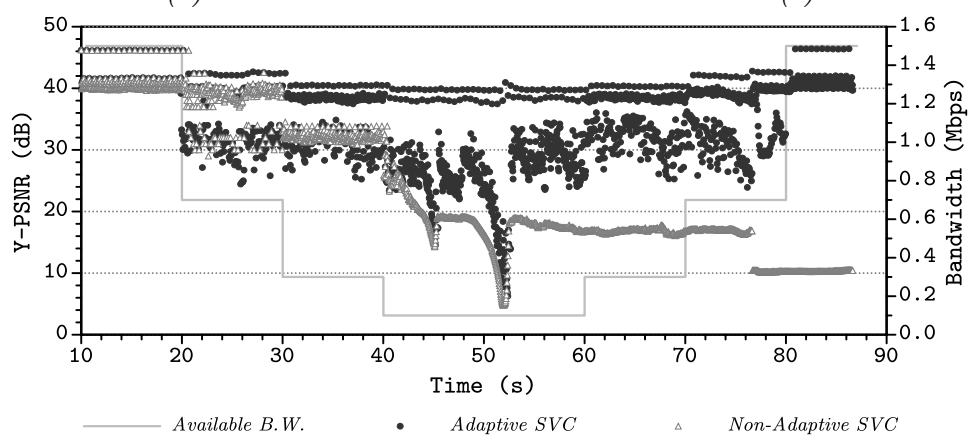

(c)

Figure 4.8. Throughput of the video flows (a), Lost Packet Rate (b), and Y-PSNR (c) for Scenario1 
Table 4.4. Average values of the results for the scenario 1

\begin{tabular}{|c|c|c|c|c|c|}
\hline \multirow[b]{2}{*}{$\begin{array}{c}\text { Time } \\
\text { Interval } \\
\text { (s) }\end{array}$} & \multirow[b]{2}{*}{$\begin{array}{c}\text { SVC layer } \\
\text { using } \\
\text { Adaptive SVC }\end{array}$} & \multicolumn{2}{|c|}{ Lost Packet Rate (\%) } & \multicolumn{2}{|c|}{ Y-PSNR (dB) } \\
\hline & & $\begin{array}{l}\text { Adaptive } \\
\text { SVC }\end{array}$ & $\begin{array}{l}\text { Non- } \\
\text { Adaptive } \\
\text { SVC }\end{array}$ & $\begin{array}{l}\text { Adaptive } \\
\text { SVC }\end{array}$ & $\begin{array}{c}\text { Non- } \\
\text { Adaptive } \\
\text { SVC }\end{array}$ \\
\hline $10-20$ & $\mathrm{BL}+\mathrm{T}_{4} \mathrm{Q}_{3}$ & 0.1 & 0.1 & 40.8 & 40.8 \\
\hline $20-30$ & $\mathrm{BL}+\mathrm{T}_{3} \mathrm{Q}_{2}$ & 0.0 & 31.9 & 35.8 & 36.4 \\
\hline $30-40$ & $\mathrm{BL}+\mathrm{T}_{3} \mathrm{Q}_{1}$ & 0.0 & 64.7 & 34.9 & 32.2 \\
\hline $40-60$ & $\mathrm{BL}+\mathrm{T}_{1} \mathrm{Q}_{0}$ & 0.0 & 90.5 & 27.5 & 18.3 \\
\hline $60-70$ & $\mathrm{BL}+\mathrm{T}_{3} \mathrm{Q}_{1}$ & 0.0 & 67.2 & 35.1 & 16.8 \\
\hline $70-80$ & $\mathrm{BL}+\mathrm{T}_{3} \mathrm{Q}_{2}$ & 0.0 & 42.4 & 35.8 & 14.7 \\
\hline $80-86$ & $\mathrm{BL}+\mathrm{T}_{4} \mathrm{Q}_{3}$ & 0.0 & 2.9 & 40.9 & 10.3 \\
\hline
\end{tabular}

However, as can be seen in the Figure 4.8a, the available bandwidth decreases and this fact creates the need to adjust the transmission rate of the video application in order to avoid the network congestion. For instance, for the intervals 30-40 s and 60-70 s the available bandwidth is $300 \mathrm{Kbps}$ and the video source maximum can transmit the layer $\mathrm{T}_{3} \mathrm{Q}_{1}$ and its dependent lower layers (see Table 4.4). Because of this, the $100 \%$ of the packets are successfully transmitted but the video is decoded with a lower quality (an average Y-PSNR of $35 \mathrm{~dB}$ for these intervals).

When a video source does not adapt its data rate (Non-Adaptive SVC curves in the Figure 4.8) some packets are lost. In the case of the segments from 30 to $40 \mathrm{~s}$ and from 60 to 70 $\mathrm{s}$, the percentages of lost packets in these intervals are of $65 \%$ and $67 \%$ respectively. This high rate of dropped packets significantly affects the quality of the received video.

When the available bandwidth drastically decrease to $100 \mathrm{Kbps}$ (interval from $40 \mathrm{~s}$ to 60 s) the layer $T_{1} Q_{0}$ is the highest layer that can be transmitted (see Table 4.4), according to the video properties described in the final part of section 4.4 (see Figure 4.7b). As illustrated Figure 4.8a and Figure 4.8b, the mechanisms of the SVCEval-RA framework allow the video source to adjust its bit rate avoiding network congestion. Thus, there were no loss packets during this segment and therefore the average Y-PSNR obtained is about $27.5 \mathrm{~dB}$ (see Figure 4.8c). In contrast, using Non-Adaptive SVC, the percentage of packet loss reaches the $90 \%$. Therefore, the quality of the received video drastically decrease and an average Y-PSNR of $18 \mathrm{~dB}$ is obtained (about $11 \mathrm{~dB}$ of difference with Adaptive SVC). Note that, the high loss packet rate from time instant $40 \mathrm{~s}$ significantly affect the decoding of the frames transmitted subsequently. In addition, the frames transmitted near the time instant 52s have a significant decrease in their PSNR values due to the high level of motion that these frames have, such as is explained in the Figure 4.9. This figure outlines the motion variance over time for the test video sequence. The motion level is calculated using Sum of Absolute Difference (SAD) between the two frames and it is given by Equation (6).

$$
S A D_{n, m}=\sum_{i=1}^{N} \sum_{j=1}^{M}\left|B_{n}(i, j)-B_{m}(i, j)\right|
$$




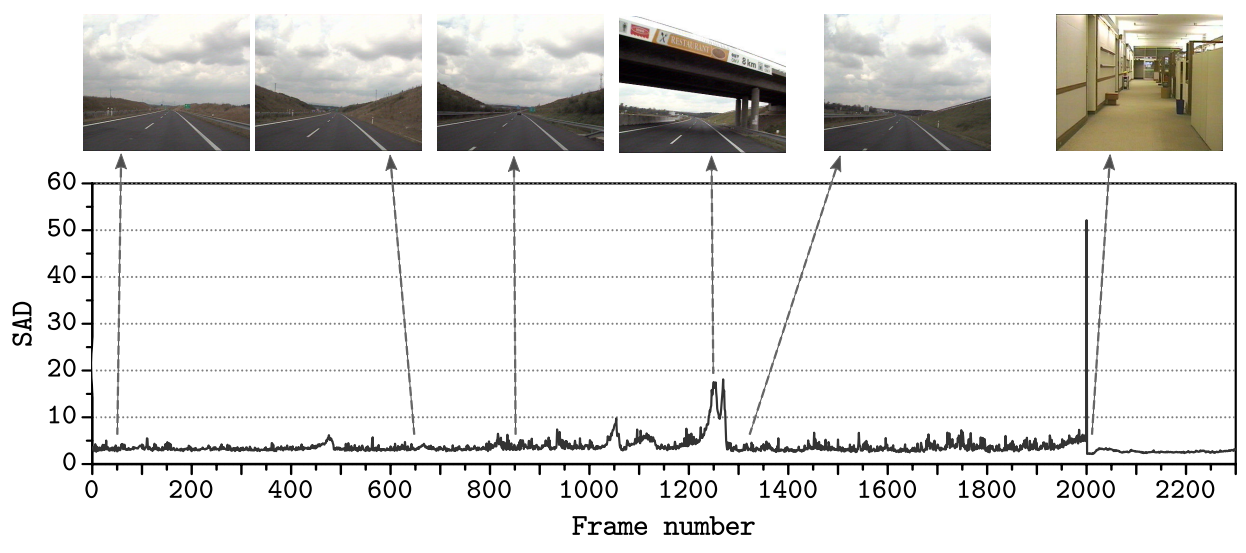

Figure 4.9.SAD estimation of the test sequence

Where $B_{n}$ and $B_{m}$ are the two frames of size $N x M$, and $i$ and $j$ denote pixel coordinates. As seen in Figure 4.9, the video sequence from frame 1 to frame 1240 is very similar. However, the spatio-temporal complexity of the frames 1240-1270 is incremented because of the changes in the foreground of the scene. Similarly, there is a high value of the SAD in the frame 2000 because of the abrupt transition that occurs when the "highway" video ends and starts the concatenated video sequence ("hall" video).

\subsection{2.b. Scenario 2: "Positive" peaks in the available bandwidth}

The second scenario was intended to test the behaviour of the adaptation algorithms of SVCEval-RA under high bandwidth peaks of different durations. Figure 4.10 represents the performance of the SVCEval-RA platform in terms of throughput, lost packet rate, and $Y$-PSNR when the network scenario produce short-term variations of the available bandwidth. In the Table 4.5 is described the transmitted layers and the average values of the results for each intervals time.

The results demonstrate that the rate-adaptive scheme implemented in the SVCEval-RA Traffic Agent has a significant rapid response to bandwidth fluctuations. Consequently, the throughput achieved using the Adaptive SVC scheme is close to the throughput achieved by the non-adaptive scheme. This rapid reaction is illustrated at $\mathrm{t}=15 \mathrm{~s}$ when there was a sudden increase in the bandwidth and the adaptation of the bit rate was immediately performed by the Traffic Agent (Figure 4.10a). Although the duration of the first bandwidth peak is only two seconds, the Adaptive SVC flow can rapidly adapt its bit rate, achieving a similar throughput to the one achieved by the non-adaptive flow, which always is been transmitted with all $S V C$ layers (i.e. to the maximum bit rate). Adaptation was also successfully performed in the following three peaks. Although at the start time of the bandwidth peaks (time instant $t=15,35,54$ and $70 \mathrm{~s}$ ) the rate adaptive scheme of the proposed framework have a conservative behaviour since they perform a slow increasing of the bit rate, this behaviour helps to avoid a buffer overload when an abrupt increasing of the available bandwidth is occurred. 


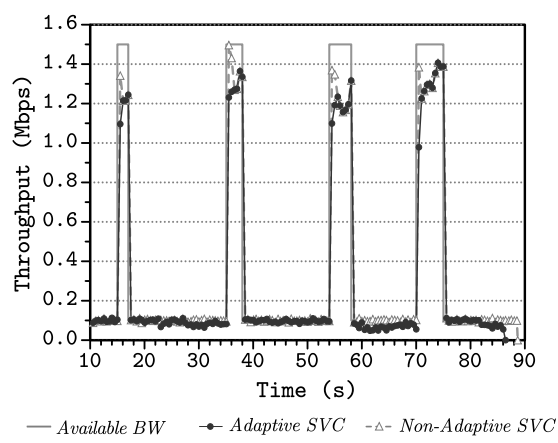

(a)

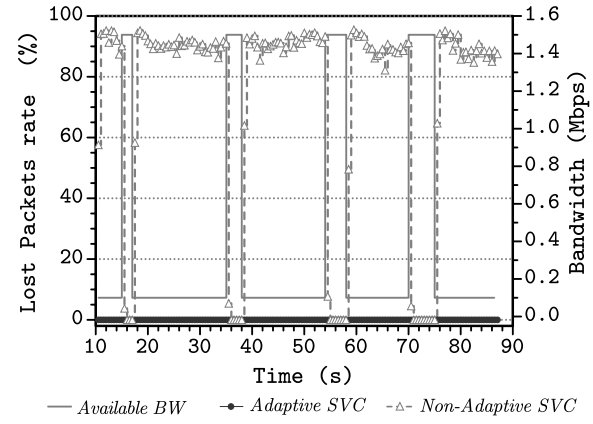

(b)

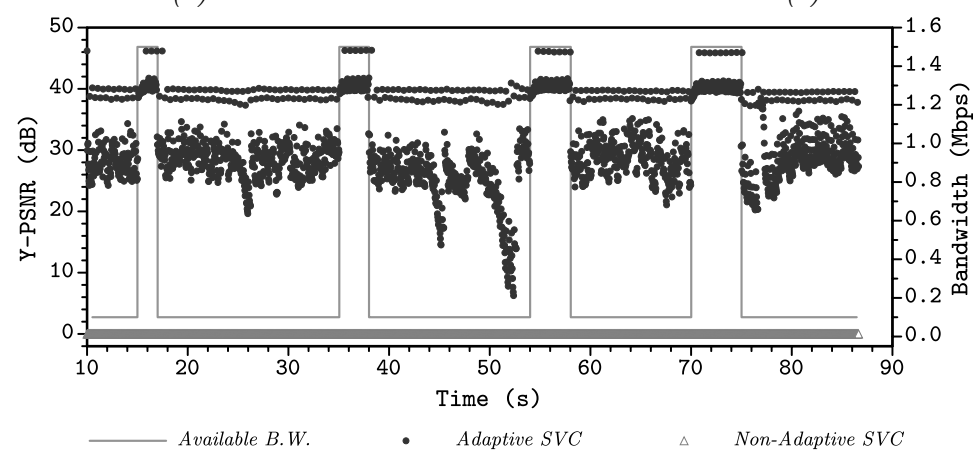

(c)

Figure 4.10. Throughput of the video flows (a), Lost Packet Rate (b), and Y-PSNR (c) for Scenario2 ("Positive" peaks in the available bandwidth)

Table 4.5. Average values of the results for the scenario 2

\begin{tabular}{|c|c|c|c|c|c|}
\hline \multirow[b]{2}{*}{$\begin{array}{c}\text { Time } \\
\text { Interval } \\
\text { (s) }\end{array}$} & \multirow[b]{2}{*}{$\begin{array}{c}\text { SVC layer } \\
\text { using } \\
\text { Adaptive SVC }\end{array}$} & \multicolumn{2}{|c|}{ Lost Packet Rate (\%) } & \multicolumn{2}{|c|}{ Y-PSNR (dB) } \\
\hline & & $\begin{array}{c}\text { Adaptive } \\
\text { SVC }\end{array}$ & $\begin{array}{l}\text { Non- } \\
\text { Adaptive } \\
\text { SVC }\end{array}$ & $\begin{array}{l}\text { Adaptive } \\
\text { SVC }\end{array}$ & $\begin{array}{c}\text { Non- } \\
\text { Adaptive } \\
\text { SVC }\end{array}$ \\
\hline $10-15$ & $\mathrm{BL}+\mathrm{T}_{1} \mathrm{Q}_{0}$ & 0.00 & 89.07 & 29.61 & 0.00 \\
\hline $15-17$ & $\mathrm{BL}+\mathrm{T}_{4} \mathrm{Q}_{3}$ & 0.00 & 23.47 & 39.84 & 0.00 \\
\hline $17-35$ & $\mathrm{BL}+\mathrm{T}_{1} \mathrm{Q}_{0}$ & 0.00 & 89.77 & 29.74 & 0.00 \\
\hline $35-38$ & $\mathrm{BL}+\mathrm{T}_{4} \mathrm{Q}_{3}$ & 0.00 & 16.07 & 40.29 & 0.00 \\
\hline $38-54$ & $\mathrm{BL}+\mathrm{T}_{1} \mathrm{Q}_{0}$ & 0.00 & 87.78 & 26.73 & 0.00 \\
\hline $54-58$ & $\mathrm{BL}+\mathrm{T}_{4} \mathrm{Q}_{3}$ & 0.00 & 12.62 & 40.51 & 0.00 \\
\hline $58-70$ & $\mathrm{BL}+\mathrm{T}_{1} \mathrm{Q}_{0}$ & 0.00 & 84.51 & 29.91 & 0.00 \\
\hline $70-75$ & $\mathrm{BL}+\mathrm{T}_{4} \mathrm{Q}_{3}$ & 0.00 & 9.57 & 40.29 & 0.00 \\
\hline $75-86$ & $\mathrm{BL}+\mathrm{T}_{1} \mathrm{Q}_{0}$ & 0.00 & 84.78 & 29.96 & 0.00 \\
\hline
\end{tabular}

On the other hand, regarding the duration of the peaks, a significant influence has not been found in this scenario since the algorithms of the SVCEval-RA Traffic Agent allowed the video source to adapt its bit rate during the shorter as well as the longer peaks. Similarly to the scenario 1, the accurate adaptation of the data rate using Adaptive SVC 
scheme avoid congestion during the intervals in which the available bandwidth is more restrictive (see Figure $4.10 \mathrm{~b}$ ). Therefore, the packet loss remains equal to 0 in the case of the Adaptive SVC flow and the average $Y$-PSNR in theses intervals is about $29 \mathrm{~dB}$. On the contrary, when the non-adaptive scheme is used the high percentage of packet loss (about $90 \%$ during the intervals of lower available bandwidth) significantly impact the number of decodable frames and therefore, received video cannot be successfully decoded (it is illustrated as Y-PSNR equal to cero in the Figure 4.10c).

\subsection{2.c. Scenario 3: "Negative" peaks in the available bandwidth}

The third scenario was intended to test the behaviour of the adaptation algorithms under low bandwidth-peaks of different durations. Figure 4.11 illustrated the behaviour of the adaptive SVC scheme of SVCEval-RA and the Table 4.6 summarizes the mean values of the results as well as the layers sent for each segment in which has been performed a variation of the available bandwidth.

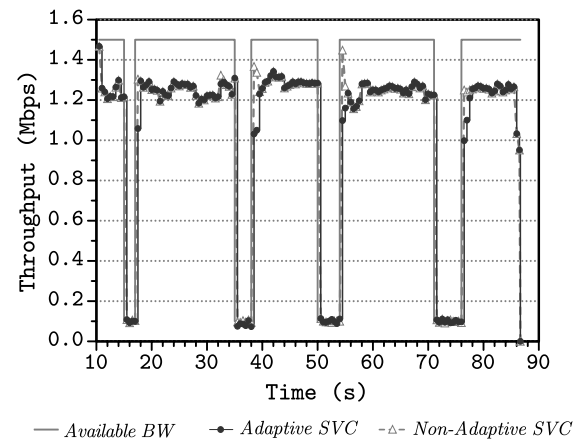

(a)

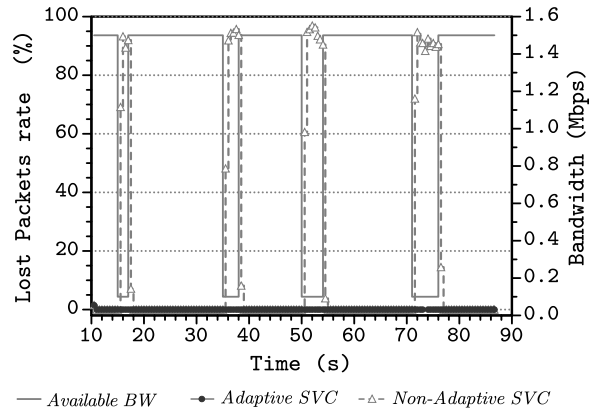

(b)

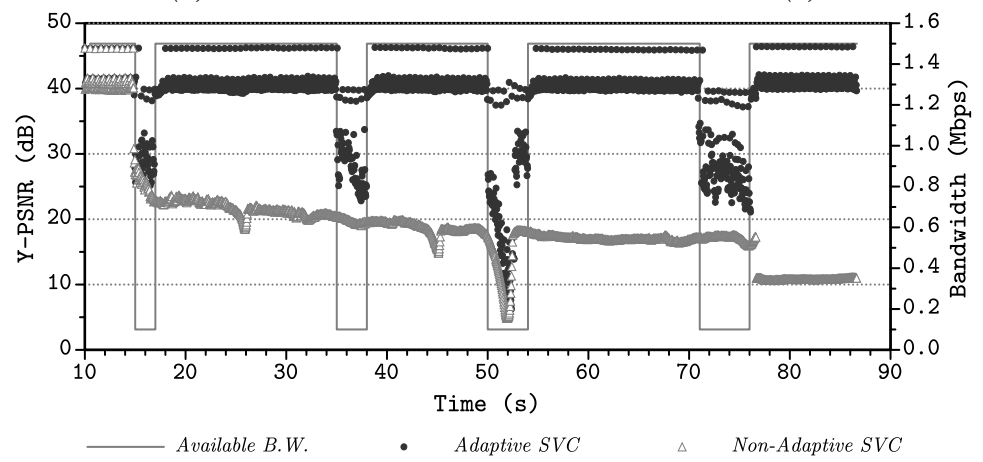

(c)

Figure 4.11. Throughput of the video flows (a), Lost Packet Rate (b), and Y-PSNR(c) for Scenario 3 (Negative peaks in the available bandwidth)

The results of the scenario 3 are consistent with the results obtained in the previous network scenarios. In all cases, when a variation of the available bandwidth was performed, the rate adaptive algorithms of the SVCEval-RA Traffic Agent allow the video source to adjust its bit rate transmitting only the $S V C$ layers that can be supported 
avoiding network congestion. Data in Table 4.6 show that when the available bandwidth is high (about $1.2 \mathrm{Mbps}$ ) the layers transmitted are the base layer and the $\mathrm{T}_{4} \mathrm{Q}_{3}$ layer whereas when the available bandwidth decreases to $0.1 \mathrm{Mbps}$ only the base and $\mathrm{T}_{1} \mathrm{Q}_{0}$ layers are transmitted. This adaptation allows us to get an important decrease in packet loss (Figure 4.11b) while it is makes more efficient use of the available bandwidth. Therefore, the quality of the received video is better than the quality achieved when the Non-Adaptive SVC scheme is used. The average Y-PSNR of the received video is $35.1 \mathrm{~dB}$ using adaptive techniques, about $15 \mathrm{~dB}$ of difference that using non-adaptive scheme. In fact, using Non-Adaptive SVC, the high rate of dropped packets during the first trough (with a duration of only 2 seconds) drastically affect the final decoding of the video (see Figure 4.11c).

Table 4.6. Average values of the results for the scenario 3.

\begin{tabular}{|c|c|c|c|c|c|}
\hline \multirow[b]{2}{*}{$\begin{array}{c}\text { Time } \\
\text { Interval } \\
\text { (s) }\end{array}$} & \multirow[b]{2}{*}{$\begin{array}{c}\text { SVC layer } \\
\text { using } \\
\text { Adaptive SVC }\end{array}$} & \multicolumn{2}{|c|}{ Lost Packet Rate (\%) } & \multicolumn{2}{|c|}{ Y-PSNR (dB) } \\
\hline & & $\begin{array}{c}\text { Adaptive } \\
\text { SVC }\end{array}$ & $\begin{array}{l}\text { Non- } \\
\text { Adaptive } \\
\text { SVC }\end{array}$ & $\begin{array}{l}\text { Adaptive } \\
\text { SVC }\end{array}$ & $\begin{array}{c}\text { Non- } \\
\text { Adaptive } \\
\text { SVC }\end{array}$ \\
\hline $10-15$ & $\mathrm{BL}+\mathrm{T}_{4} \mathrm{Q}_{3}$ & 0.2 & 0.2 & 40.7 & 40.5 \\
\hline $15-17$ & $\mathrm{BL}+\mathrm{T}_{1} \mathrm{Q}_{0}$ & 0.0 & 63.0 & 30.5 & 25.2 \\
\hline $17-35$ & $\mathrm{BL}+\mathrm{T}_{4} \mathrm{Q}_{3}$ & 0.0 & 2.8 & 40.7 & 21.7 \\
\hline $35-38$ & $\mathrm{BL}+\mathrm{T}_{1} \mathrm{Q}_{0}$ & 0.0 & 70.8 & 29.4 & 19.7 \\
\hline $38-50$ & $\mathrm{BL}+\mathrm{T}_{4} \mathrm{Q}_{3}$ & 0.0 & 4.2 & 40.7 & 18.7 \\
\hline $50-54$ & $\mathrm{BL}+\mathrm{T}_{1} \mathrm{Q}_{0}$ & 0.0 & 78.8 & 23.8 & 13.8 \\
\hline $54-71$ & $\mathrm{BL}+\mathrm{T}_{4} \mathrm{Q}_{3}$ & 0.0 & 2.8 & 40.6 & 17.1 \\
\hline $71-76$ & $\mathrm{BL}+\mathrm{T}_{1} \mathrm{Q}_{0}$ & 0.0 & 80.0 & 28.5 & 17.2 \\
\hline $76-86$ & $\mathrm{BL}+\mathrm{T}_{4} \mathrm{Q}_{3}$ & 0.0 & 4.6 & 40.6 & 11.2 \\
\hline
\end{tabular}

Regarding the effects of the duration of the troughs, has not been found an influence over the efficacy of the algorithms implemented in the Traffic Agent of SVCEval-RA. In all case, the Traffic Agent provides a rapid response to adapt the transmission rate to abrupt decrease of the available bandwidth even though the duration of the troughs is short, such as is the case of the first "negative" peak.

\subsubsection{Conclusions}

In general terms, the obtained results prove that the proposed framework provides efficient network-adaptive algorithms to perform realistic network simulations that involve adaptive video streaming supporting SVC coding. The Adaptive SVC scheme presented in the proposed framework allows the traffic source to transmit only the SVC layers that can be efficiently supported by network. This fact provides better conditions to video streaming as demonstrated by the Y-PSNR obtained in the simulation study. Using SVCEval-RA platform a feedback about the current network status is provided to the source application in order to set the layers that can be transmitted. Without this information, the video may not be adapted, causing congestion in the network and a large number of dropped packets. 


\subsection{Adaptive video transmission using AQA-AODV}

After introducing the simulation platform to be used, in this section the idea of evaluating the transmission of scalable video using AQA-AODV, described in previous chapter, is taken up again. As in the simulations tests presented in Chapter 3, the performance of AQA-AODV is compared with AODV and QAODV [69]. The objective of the simulation study is to demonstrate that the proposed solution is an effective system for providing video streaming services over MANETs.

\subsubsection{Video analysis}

The traffic flow used in the simulations consists of a video stream, which has been created by concatenating the well-known test sequence SINTEL TRAILER [107] with a resolution of 1280x720 pixels (720p Format and 16:9 aspect ratio) to form a testing video of 2506 frames. The video sequence has been encoded according to H.264/SVC standard with two types of scalability: temporal and quality. As SVC codec, the JSVM codec was used [103]. All the values for the video related parameters are reported in Table 4.7.

Table 4.7. Video Parameters

\begin{tabular}{l|l}
\hline \hline Parameters & Description/Value \\
\hline Original Video file & YUV format \\
Size & 2506 frames \\
Frame per second & 24 \\
Duration & $104.4 \mathrm{~s}$ \\
Encoded video file & H.264/SVC \\
Type of scalability & SNR (MGS) \\
GOP Size & 16 frames \\
\hline
\end{tabular}

The video sequence was encoded in five temporal layers (from $T_{0}$ to $T_{4}$ ). At the same time, three extra levels of quality scalability (from $Q_{0}$ to $Q_{3}$ ) were added at each temporal level. For quality scalability was used the Medium Grain Quality Scalability (MGS). The use of MGS layers for quality scalability allows source video to discard the data units from the enhancement layers without affecting the result bit-stream.

Figure 4.12a gives a graphical description of the bit rates obtained according to the temporal levels and the MGS layers. The labels on the bars indicates the layer id assigned by the SVC encoder. In total, twenty video layers (from $L_{0}$ to $L_{19}$ ) were obtained from the combination of sublayers $T_{i}$ and $Q_{j}$. The Y-axis in Figure 4.12a indicates the bit rate associated to each layer. Depending of the number of transmitted layers, the output bit rate varies from $79.4 \mathrm{kbps}$ (sending Layer $L_{0}$ alone) to $775.7 \mathrm{Kbps}$ (sending Layers 0 to 19). These values are aggregated, which means that to transmit Layer $L_{3}\left(T_{3} Q_{0}\right)$ also dependent lower layers have to be transmitted, i.e., layers $L_{0}, L_{1}$, and $L_{2}$. Therefore, the total bandwidth required would be of $202 \mathrm{Kbps}$. 


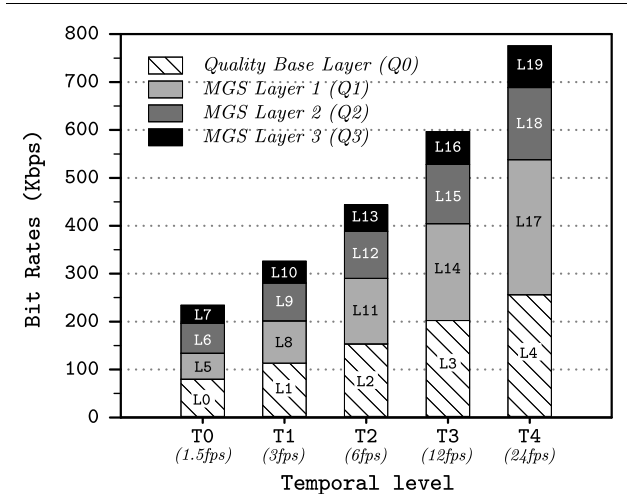

(a)

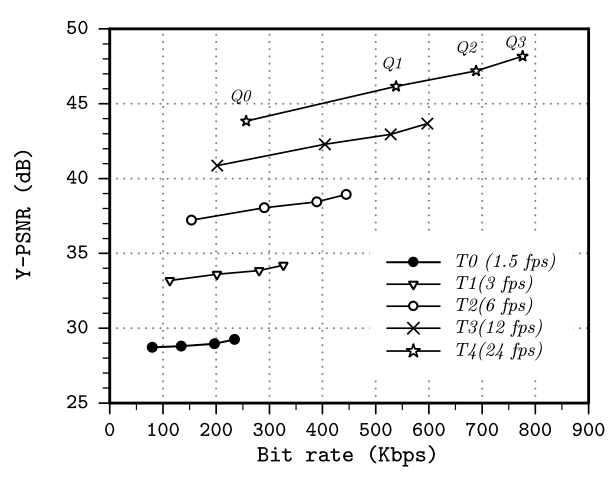

(b)

Figure 4.12. Description of the SVC layers contained in the video stream (a) and Rate-distortion analysis of the $S V C$ video stream $(b)$

Moreover, a rate-distortion analysis in terms of average Y-PSNR (PSNR for the luminance component in the YUV colour space) versus average bit rate was computed off-line (see Figure 4.12b). The computation of the Y-PSNR curves were performed by stripping out the layers, measuring the average bit rate, decoding the resulting video, and computing the average Y-PSNR. Each of these curves represents a temporal layer and each point corresponds to a MGS layer (from $Q_{0}$ to $Q_{3}$ ). This figure describes the increase in the video quality (in terms of Y-PSNR) depending on the number of quality and temporal layers that make up the video.

\subsubsection{Simulation environment}

The simulation experiments were performed in the SVCEval-RA framework (described in previous chapter) with IEEE802.11 protocol in the MAC layer working in the distributed coordination function (DCF) mode with a channel data rate of $2 \mathrm{Mbps}$. The queue type is Drop Tail with maximum length of 50 packets. The most relevant simulation parameters are shown in Table 4.8.

Table 4.8. Simulation Parameters

\begin{tabular}{l|l}
\hline \multicolumn{1}{c|}{ Parameter } & \multicolumn{1}{c}{ Value } \\
\hline MAC Protocol & 802.11 \\
Propagation model & Two Ray Ground \\
Queue type & Drop Tail \\
UDP packet size & 1000 bytes \\
Transmission range & $250 \mathrm{~m}$ \\
Interference range & $550 \mathrm{~m}$ \\
\hline
\end{tabular}

\subsubsection{Simulation Scenarios}

Two simulation scenarios were evaluated. The first scenario, called "roaming node", is depicted in Figure 4.13. It consists of eleven nodes: ten are stationary nodes $(1,2,3, \ldots, 10)$ and one node (node 0 ) is a mobile node ("roaming" node). The transmission range and interference range are $25 \mathrm{~m}$ and $55 \mathrm{~m}$ respectively so that nodes only have connectivity 
to their adjacent neighbours. In this scenario, the video source (node 0 ) and the destination node (node 1) always have connectivity through one or more hops. The labels $a, b, c, d, \ldots j$ correspond to the physical locations of the nodes. Node 0 starts moving from the initial position and every 10 seconds it reaches the next position $(a, b, c, \ldots, j)$. The movement will force node 0 to use the nodes from 2 to 10 to relay its data packets. The effects of both mobility and the number of hops over the bit rate adaptation is studied in this scenario.

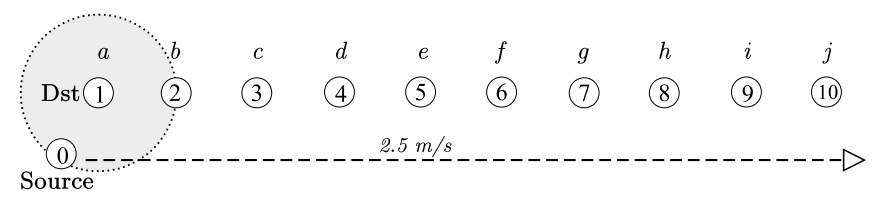

Figure 4.13. Scenario 1: "roaming node". Video Source is node 0 and the destination is node 1.

The second scenario is a more realistic scenario where 30 mobile nodes move in a $1000 \mathrm{~m}$ $\mathrm{x} 1000 \mathrm{~m}$ area according to the random waypoint model. The video traffic is established between a random source-destination pair. In addition to video traffic, some CBR (Constant Bit Rate) flows were also applied as background traffic. The aim of this scenario is to evaluate the influence of node movement on the performance of the adaptation algorithms of AQA-AODV and on the quality of video transmission.

For each type of scenario, the simulation was run for 10 times (with random scenarios with different seeds) to take average values in the measured performance metrics. The results are obtained with a confidence level of $95 \%$.

\subsubsection{Video performance metrics}

With the aim of evaluating the video transmission over AQA-AODV protocol, PSNR, Packet loss rate and end-to-end packet delay were chosen to measure the quality of the received video sequence. These parameters are tightly related to QoS. Similarly, the Peak Signal-to-Noise Ratio (PSNR) was used to evaluate the difference between the received sequence and the original one pixel by pixel. To correctly estimate the PSNR, it is necessary to establish a synchronization mechanism between sent and received frames. This is required since the frames may be lost during transmission due to congestion, or because the adaptive rate controller considered appropriate not to send some layers. For example, when video source only transmits the layer $0\left(\mathrm{~T}_{0} \mathrm{Q}_{0}\right)$, the computation of the PSNR must take into account the lack of the frames belonging to other layers. Otherwise, the calculation would not be correct since the frames that are being compared do not match each other.

Figure 4.14a shows a basic example where the sent and received frames are not synchronized and therefore the PSNR calculation is wrong. The correct method (shown in Figure 4.14b) involves duplicating the frames of the lower layer so that both sequences have the same number of frames. Additionally, another performance metric is defined, namely decodable frame rate. The decodable frame rate is an application-level metric, which is defined as the ratio of the number of successfully decoded frames over the total 
number of frames. As simulations presented in Chapter 3, AODV and QAODV protocols were also evaluated with the aim of evaluating their performance and compare them with AQA-AODV.

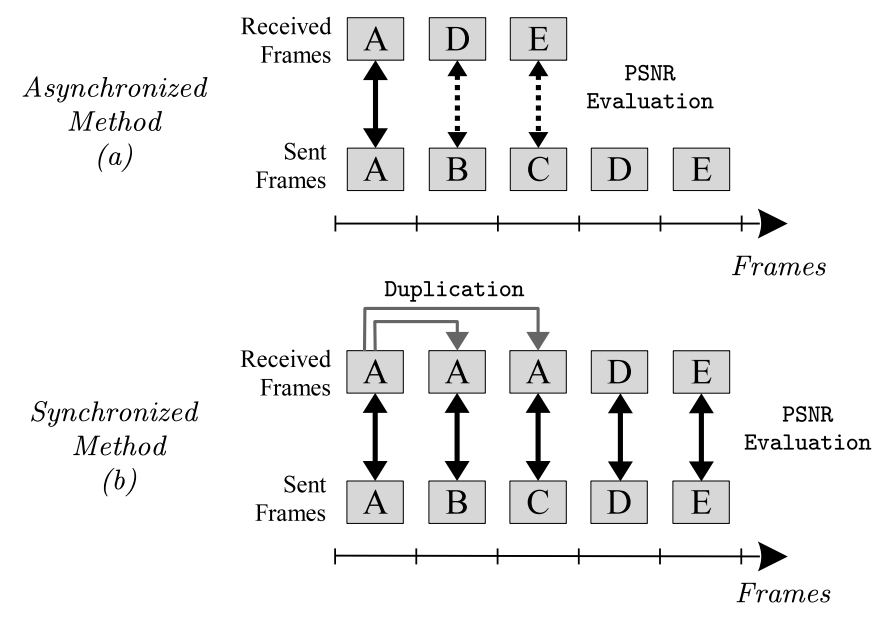

Figure 4.14. Synchronization of frames during the PSNR Calculation.

\subsubsection{Simulations Results}

\subsection{5.a. Scenario 1: static linear topology with a mobile node (roaming node)}

In the first network scenario, the performance of AQA-AODV was tested as function of the number of hops on the path. Initially the source requested a transmission rate of $0.775 \mathrm{Mbps}$, which be maintained constant when AODV and QAODV are used. However, using AQA-AODV, this transmission rate can be changed by the source because of the adaptive feedback scheme. The data rate of $0.775 \mathrm{Mbps}$ is the required rate to transmit all SVC layers and achieve the maximum video quality, such as was described in Section 4.5.1 (see Figure 4.12).

In Figure 4.15 is depicted the variation of the Available Bandwidth (BWav) as the number of hops is increased. This estimation was calculated by the algorithm implemented in AQA-AODV, which is consistent with the study presented in Chapter 2. In addition to the available bandwidth curve, in Figure 4.15 also is presented the total network throughput achieved using the three evaluated protocols. This figure shows that the throughput achieved with AQA-AODV is slightly lower to the throughput achieved using AODV and very close to the available bandwidth. As source moves away from the destination node, increasing the number of hops, the video source adapts its data rate transmitting only the layers that can be supported by the route. In any case, the throughput exceeds the effective available bandwidth, avoiding network congestion. In contrast, using a conventional technique of transmission in MANETs (such as AODV), the source does not know the available bandwidth and it injects packets to the network (during the entire simulation) with a fixed rate of $0.775 \mathrm{Mbps}$ without adaptation. This 
data rate only can be supported during the first 10 seconds of the simulation (i.e, when the route has 1 hop of length) such as is shown by the $B W a v$ curve. Therefore, there is a significant increase in network congestion and packet loss as the number of hops increases (see Figure 4.16).

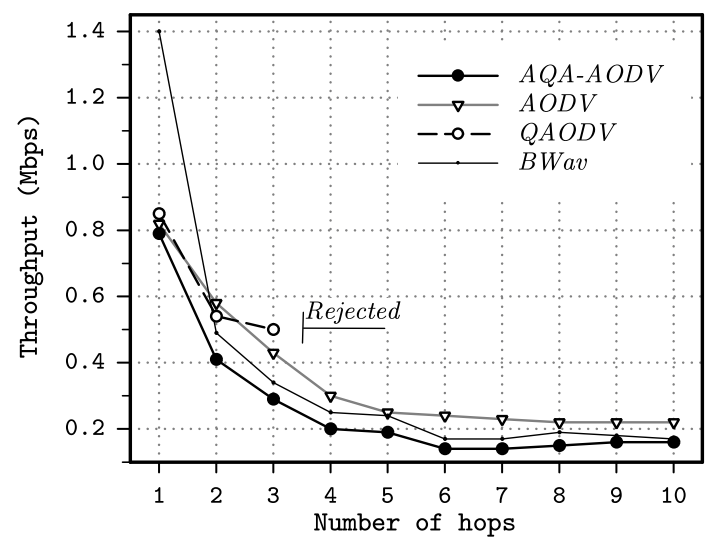

Figure 4.15. Available Bandwidth and Throughput achieved during simulation as a function of the number of hops.

The results of AODV show that source node sends more packets to the network exceeding the available bandwidth of the route. However, a high percentage of these packets are eliminated due to congestion. In contrast, using AQA-AODV the source sends packets to the network with a lower rate; therefore, network congestion is significantly reduced. In other words, a significant decrease in the rate of lost packets (Figure 4.16) and the average delay (Figure 4.17) is reached as a result of the adaptive feedback scheme and the route recovery mechanism of AQA-AODV. This fact provides better conditions to video streaming applications as demonstrated by the results of the video evaluation, which will be discussed later.

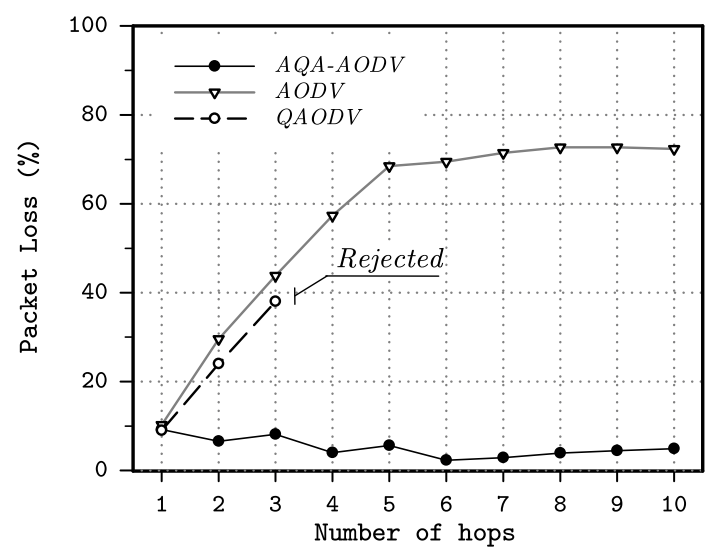

Figure 4.16. Packet Loss as a function of the route length 


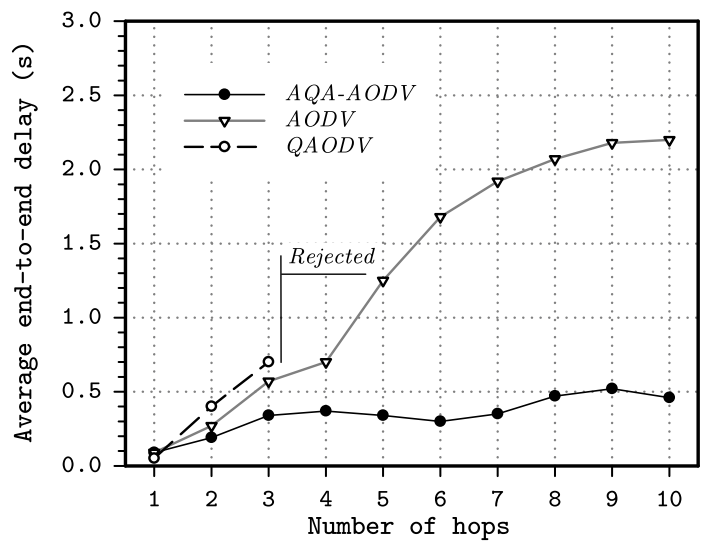

Figure 4.17. Average end-to-end delay during the network simulation

Regarding QAODV, its admission control system only accepts the traffic flow of 0.775 Mbps when the number of hops between source and destination is less than 3 . In these cases, QAODV presents a similar behaviour to AODV in terms of throughput, delay and packet loss. In the figures, the label "rejected" was added on the curves of QAODV in order to denote that the traffic flow is rejected by the admission control system when the route cannot support the requirements of the traffic flow.

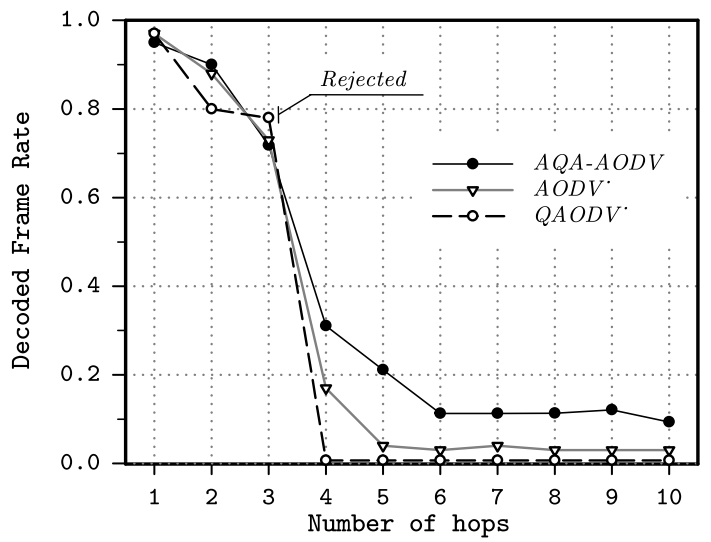

Figure 4.18. Decoded frame rate as function of the number of hops

Concerning video transmission, Figure 4.18 illustrates the decoded frame rate and Figure 4.19 shows the average PSNR obtained during the network simulation. As seen in these figures, when the route has one hop, the quality of the received video is close to the maximum value that can be reached. This fact is due to highest layer $\left(L_{19}\right)$ and its dependent lower layers can be supported by the route capacity. However, as can be seen in Figure 4.15, the available bandwidth decreases as the number of hops increases. Therefore, video application should adjust the transmission rate in order to avoid network congestion. For example, when there are two or three hops between source and destination, using the proposed solution (SVC/AQA-AODV) the video application adjusts its data rate avoiding a high rate of dropped packets. Nevertheless, only a slight 
difference in the Y-PSNR is obtained with AODV and QAODV. Although some packets were lost in this segment of the simulation (mainly using AODV and QAODV), the robustness of the layered scheme of SVC provides an effective compensation.

As observed in Figure 4.18, for the segments of the simulation where the route has four or five hops, the decoded frame rate drastically decreases for the three protocols. In the case of AQA-AODV, this reduction is mainly caused by bit-rate adaptation performed by the video application, which sends only the packets belonging to the layers that can be supported by the route. For instance, the available bandwidth when the route has 4 hops is $250 \mathrm{Kbps}$; therefore, the video application only sends the data of the layer $L_{3}\left(T_{3} Q_{0}\right)$ and its dependent lower layers. Layer $L_{2}\left(T_{2} Q_{0}\right)$ and its dependent lower layers are sent when there are five hops ( $240 \mathrm{Kbps}$ of $B W a v$ ) in the route. In the case of AODV, the low rate of decoded frames is caused by the high rate of lost packets and the number of packets that have been discarded by SVCEval-RA tool after the play-out buffer deadline due to the high transmission delay. Using QAODV, its admission control rejects the traffic flow and the video transmission is cancelled. In any case, the decoded frame rate is always higher with AQA-AODV, about $50 \%$ higher than AODV. Moreover, Figure 4.19 clearly shows that AQA-AODV provides the highest video quality (an average Y-PSNR of $34 \mathrm{~dB}$ for 4 hops and $32 \mathrm{~dB}$ for 5 hops). When the route has six hops o more, the available bandwidth is more restrictive but network congestion is avoided due to the accurate adaptation of the data rate using the combination of the adaptive SVC technique and AQA-AODV. For this segment of the simulation, the highest SVC layer transmitted by the video source is the layer $L_{3}$, which has an aggregate bit rate of $202 \mathrm{Kbps}$ according to the analysis presented in Figure 4.12. Consequently, a larger number of frames are successfully decoded improving the quality of the received video in $10 \mathrm{~dB}$ in the worst case, compared to AODV (see Figure 4.19).

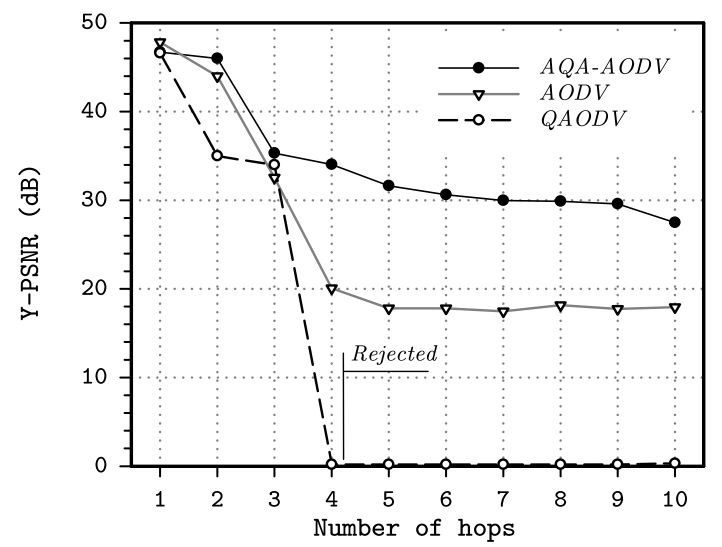

Figure 4.19. Average Y-PSNR as function of the number of hops

In general terms, the results of this first network scenario demonstrate that the combination of the adaptive SVC scheme and the QoS mechanisms of AQA-AODV provides an efficient network-adaptive strategy. The adaptive scheme implemented in the proposed solution allows the traffic source to transmit only the SVC layers that can 
be efficiently supported by network. This fact provides better conditions to video streaming with an acceptable quality minimizing the pauses or video gaps caused by losses. Using AQA-AODV feedback about the current network status is provided to the source application in order to set the layers that can be transmitted. Without this information, the video may not be adapted, causing congestion in the network and a large number of dropped packets.

\subsection{5.b. Scenario 2: mobile topology}

In order to demonstrate that the feedback scheme of AQA-AODV is an efficient method for video streaming over MANETs, a simulation study was conducted. In the simulated network scenario, 30 nodes move in a rectangular area of $1000 \mathrm{~m} \times 1000 \mathrm{~m}$ according to the random waypoint model. In this scenario the nodes starts its journey from a random location to a random destination with a randomly chosen speed (uniformly distributed between $0-5 \mathrm{~m} / \mathrm{s}$ ). Once the destination is reached, another random destination is targeted after a pause. Pause time, which affects the relative speeds of the mobiles, was varied from 0 to 120 seconds. A pause time of 0 seconds corresponds to the worst scenario because wireless nodes are all the time moving during the simulation. Transmission range for each node is $250 \mathrm{~m}$ and channel capacity is $2 \mathrm{Mbps}$. Simulations are run for 200 seconds and each data point represents an average of at least ten runs with identical traffic models, but different randomly generated mobility scenarios. Identical mobility and traffic scenarios are used across protocols. The traffic flow consists of a video stream of 2506 frames, such as was described in Section 4.5.1. In order to avoid the spatial distribution change problem, the video stream starts being transmitted after 70 seconds of simulation with a data rate of $775 \mathrm{Kbps}$, which correspond to the highest encoded bit rate of the video stream. In addition to the video flow, five flows of $10 \mathrm{Kbps}$ are introduced randomly as background traffic in the network. These traffic flows are CBR (Constant Bit Rate) over UDP.

This simulation scenario was intended to test the impact of the mobility of the nodes on the performance of AQA-AODV and on the video streaming quality. In order to evaluate the quality of the received video several measurements were done, involving network and video metrics, such as packet loss rate, delay, decoded frame rate and Y-PSNR. These parameters are related to the objective quality of the reconstructed videos. The results of the video evaluation using AQA-AODV, QAODV and AODV are shown below.

Figure 4.20 and Figure 4.21 show the results of the simulations in which the packet loss and average end to end delay are plotted versus the pause time. In terms of packet loss (Figure 4.20), AQA-AODV shows an important improvement over AODV, which reaches very high packet losses as mobility of the nodes increases. More specifically, AQA-AODV outperforms AODV by about 40 percent at lower pause times (higher mobility) and 30 percent for higher pause times. The relative performance of AODV and AQA-AODV with respect to average end-to-end delay is similar to that with packet loss rate. With AODV, the maximum average delay reaches $800 \mathrm{~ms}$ for a time pause of $0 \mathrm{~s}$ whereas using AQAAODV always is maintained a delay below $97 \mathrm{~ms}$ (about 8 times lower than using AODV). The reason of the high values of the delay and packet loss for AODV is that the established routes between the sender and receiver nodes during the simulation time 
cannot support the transmission rate of $775 \mathrm{Kbps}$. Moreover, because of the lack of QoS mechanisms in AODV that allow the video application to adapt its data rate, a high level of traffic congestion is caused.

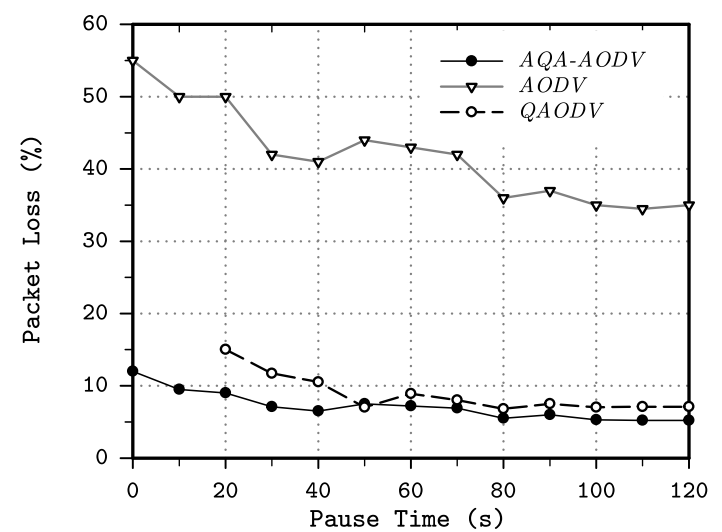

Figure 4.20. Packet Loss for a mobile scenario with different pause times

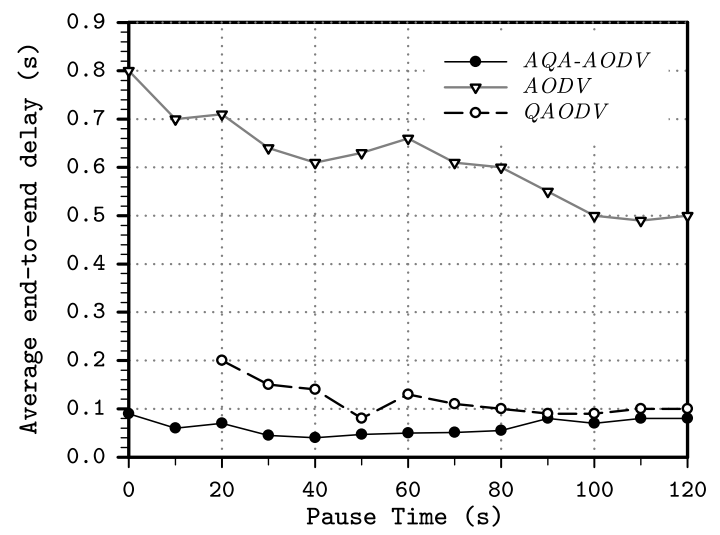

Figure 4.21. Average end-to-end delay as function of pause time

With reference to QAODV, although this protocol shows a similar performance to AQAAODV, only in very few cases the video sequence could be completely transmitted. For example, for pause times below $20 \mathrm{~s}$ the transmission of the video could not be started since the admission control of this protocol rejects the video traffic flow. For pause times above $20 \mathrm{~s}$, the transmission of the video packets is performed only for a short time interval, then QAODV rejects the traffic flow and the video transmission is cancelled. Hence, the points of the curve of QAODV represent measurements taken during the periods in which the video packets are streamed to the destination node. While the communication between source and destination is maintained, the obtained results with QAODV, in terms of packet loss and delay, seem to have a better behaviour, compared to AODV. Also can be observed a slight increase of these metrics, compared 
to AQA-AODV, which may be caused by the delay of the route recovery mechanism of QAODV, which has a worse performance than the one of AQA-AODV such as demonstrated by measurements of the CLS (Connection Setup Latency) presented in the analysis presented in Chapter 3.

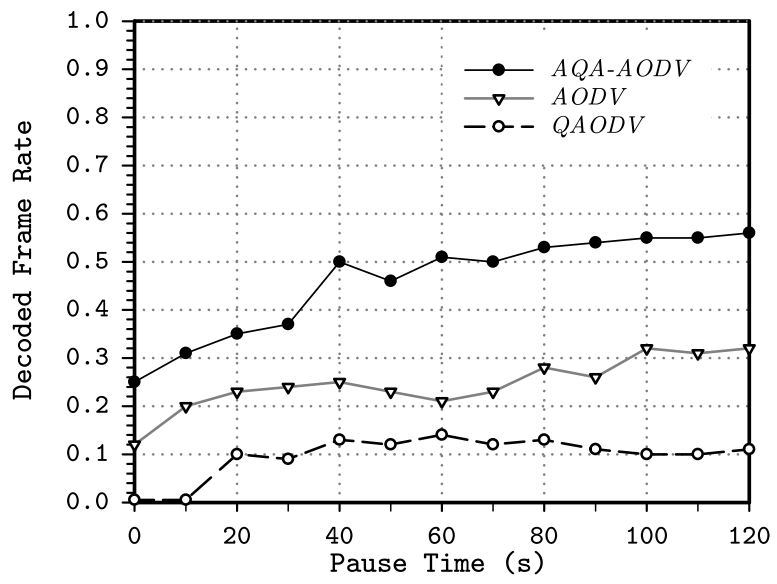

Figure 4.22. Decoded Frame Rate as a function of the pause time

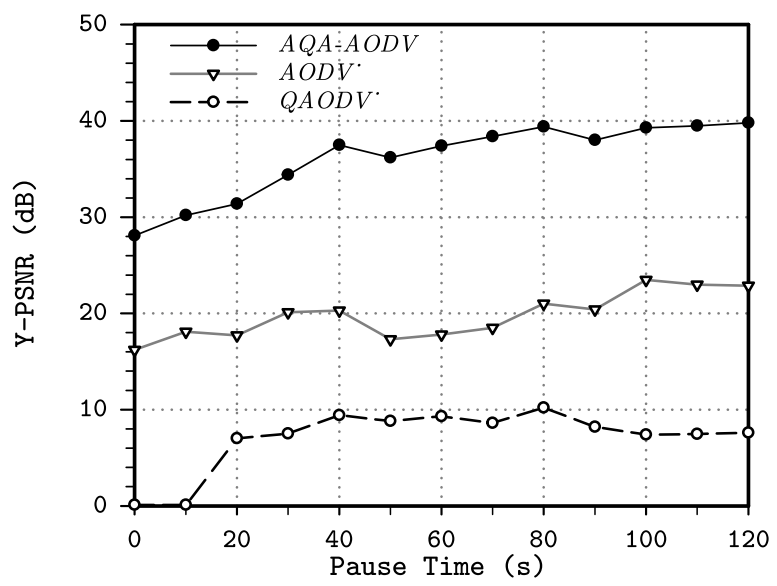

Figure 4.23. Average Y-PSNR as a function of the pause time

As far as the video quality evaluation is concerned, Figure 4.22 and Figure 4.23 report the decoded frame rate and the PSNR for the three protocols. The results in Figure 4.23 show that under all mobility levels, AQA-AODV overall outperforms AODV and QAODV. Using AQA-AODV there was a high variation of the decoded frame rate with the increase of the mobility. For example, under low mobility conditions, the decoded frame rate is 0.25 and for high mobility is 0.55 , i.e., a difference of $54 \%$. This variation is mainly a consequence of the removal process of temporal layers from the video stream, performed by the video application in order to adapt the bit rate to the network state. On the other hand, the low values for the decoded frame rates using AODV are caused 
by both $i$ ) packet losses due to erroneous transmission over the wireless ad hoc network, and $i$ ) packets discarded at the playout buffer because they were received too late at the destination node to be played out.

In order to get a better insight into how quality degradation is distributed for a given video streaming depending on the available bandwidth, a specific scenario was analysed with more detail. This sample scenario corresponds to that in which the pause time was set to 80s. Figure 4.24 reports the corresponding results for this sample scenario. The results have been divided in two parts, Figure 4.24a shows the results until frame 2250 and Figure 4.24b shows the results from frame 2251 to 2506. The top graphs in Figure 4.24a and Figure 4.24b show the PSNR per frame of the video stream as a function of the frame index. The bottom graphs illustrate the available bandwidth of the route between source and destination node. Both graphs are aligned to capture the variation of PSNR according to changes in the available bandwidth. Bandwidth curve also contains some negative spikes, which are caused by the link failures.

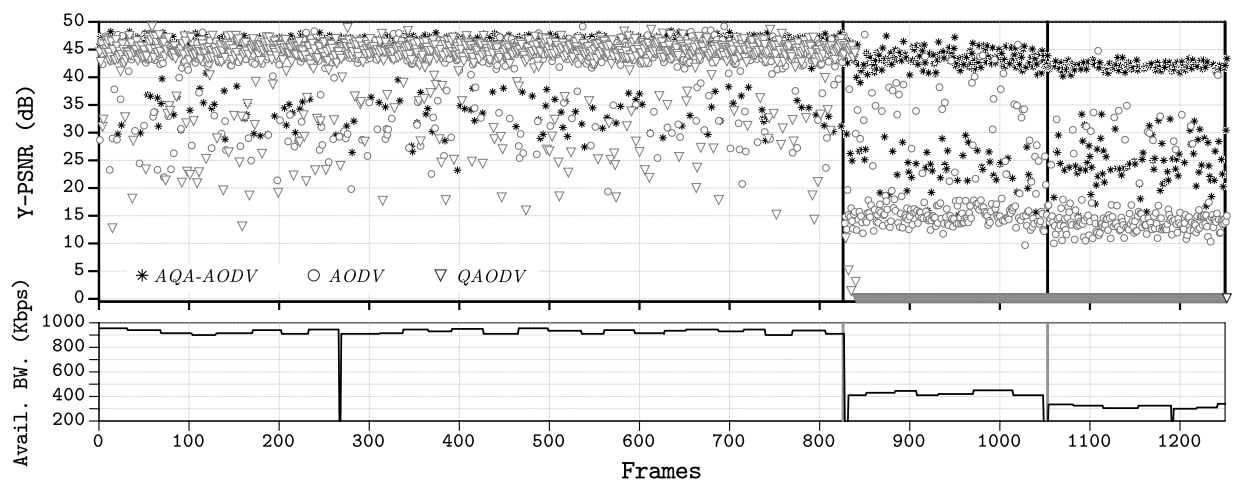

(a)

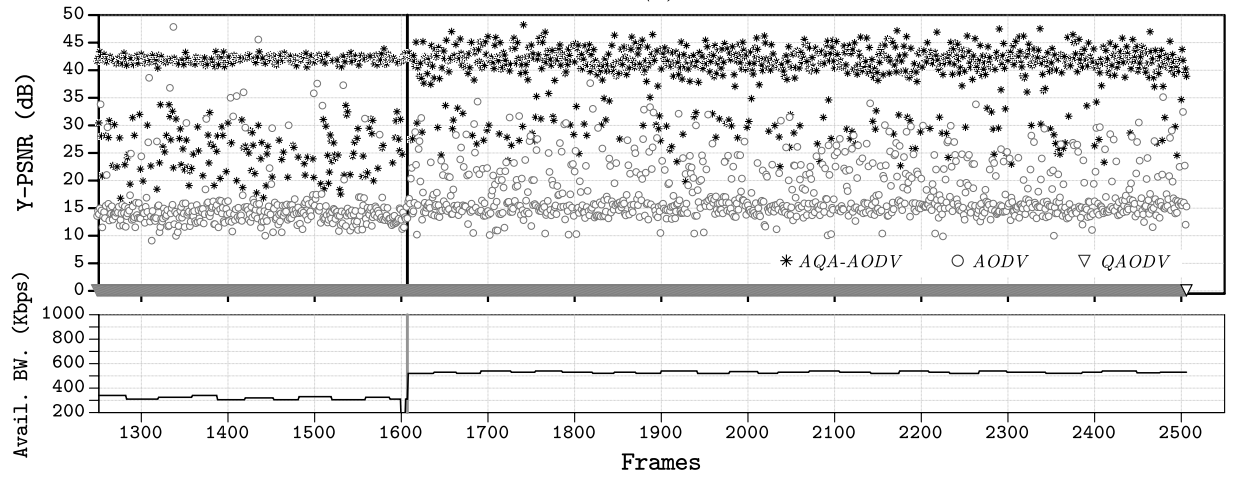

(b)

Figure 4.24. Y-PSNR and Bandwidth Available as a function of the frame index (mobile topology, pause time $=80 \mathrm{~s}$ ). Results from frame 0 to 2250 (a) and from frame 2251 to 2506 (b)

In Figure 4.24a and Figure 4.24b can be distinguished four intervals, such as indicated by the vertical markers on the graphs. In the first interval (i.e. between frames 0 and 826) the available bandwidth is above $900 \mathrm{Kbps}$; thus, all SVC layers are transmitted. This fact 
is because the bit rate required to send the highest layer and its dependent lower layers (i.e. $775 \mathrm{Kbps}$ ) can be supported by the route. For the next two intervals, the available bandwidth decreases to 400 and $200 \mathrm{Kbps}$ respectively. This is due to the mobility of the nodes and multiple access interferences at certain regions of the ad hoc network. During these two intervals, using AODV, the video source continues sending packets at a fixed rate of $775 \mathrm{Kbps}$, which leads to network congestion. Thus, the high amount of dropped packet causes a significant decrease of PSNR. On the other hand, QAODV rejects the traffic flow since it cannot support the data rate requested by the video source; therefore, video streaming is cancelled. Whereas AQA-AODV allow video application to decrease its bit rate transmitting the layer $L_{14}\left(T_{3} Q_{1}\right)$ in the second interval (from frame 826 to frame 1053) and the layer $L_{11}\left(T_{2} Q_{1}\right)$ and its dependent layers in the third interval (frames 1054 to 1607). These layers have a temporal resolution of $12 \mathrm{fps}$ and $6 \mathrm{fps}$, respectively. Due to this fact, a large amount of frames (in the second and third intervals) has a lower PSNR than the frames in the first interval, where the frame rate was $24 \mathrm{fps}$. Subsequently, during the last interval the available bandwidth up to about $530 \mathrm{Kbps}$ and video source (with AQA-AODV) increases its bit rate transmitting the layer $L_{15}\left(T_{3} Q_{2}\right)$ and its dependent layers. In contrast, with AODV only a slight improvement is obtained due to the large frame losses of the above intervals.

In order to provide a subjective measure of the QoE, the Mean Opinion Score (MOS) was used. In general, MOS is a numerical indication of the quality of the media perceived by the end user, i.e., after transmission and decoding. Since MOS is a subjective metric, its assessment requires human interpretation. However, it is very much time consuming. For this reason, usually the MOS can be approximated by estimation from a corresponding objective metric, by means of a mapping table or a formula. In this case, the mapping defined in [98] was adopted, which enables the conversion from PSNR to MOS as illustrated in Table 4.9.

Table 4.9. Possible PSNR to MOS conversion and impairment scale

\begin{tabular}{c|c|l}
\hline \hline PSNR $[\mathbf{d B}]$ & MOS & \multicolumn{1}{c}{ Impairment } \\
\hline$>37$ & 5 (Excellent) & Imperceptible \\
$31-37$ & 4 (Good) & Perceptible, but not annoying \\
$25-31$ & 3 (Fair) & Slightly annoying \\
$20-25$ & 2 (Poor) & Annoying \\
\hline
\end{tabular}

Figure 4.25 shows the cumulative distribution function of the PSNR per frame in the considered scenario. The MOS levels, derived from PSNR values as described in Table 4.9, are also highlighted. Figure 4.25 illustrates how the variation of the network conditions affects the quality of the streamed video. In particular, with AQA-AODV the amount of frames with high PSNR values (i.e., those corresponding to the Excellent MOS level) is much larger than the corresponding amount with AODV and QAODV. On the other hand, with AODV, data loss has a significant effect on quality degradation such as evidenced by the high number of frames with low PSNR values (Bad to Poor MOS levels). 


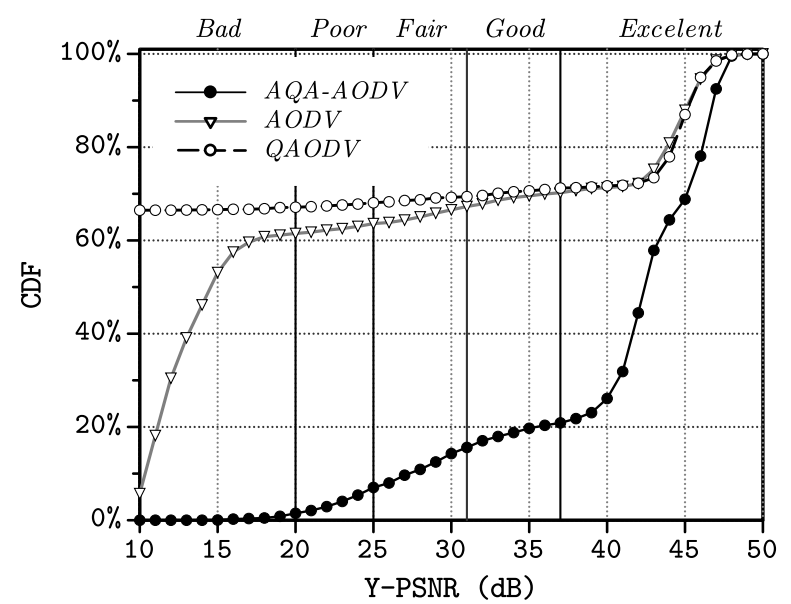

Figure 4.25. Cumulative Distribution Function (CDF) of PSNR per frame (mobile topology, pause time $=80 \mathrm{~s}$ )

\subsubsection{Conclusions}

In general terms, the results of the simulation study demonstrate that the combination of the layered scheme of SVC and the QoS mechanisms of AQA-AODV provides an efficient system for network-adaptive video streaming. The bandwidth estimation algorithms of AQA-AODV gather accurate information about the network resources. This information is then sent to the application layer of the source node in order to transmit only the SVC layers that could be efficiently supported by network. This fact helped or avoided the network congestion providing better conditions to video streaming. For example, in all simulated scenarios, the rate-adaptive strategy implemented using SVC and AQA-AODV shows better performance in terms of packet loss, end-to-end delay and video quality. It is important to note that, even though with AQA-AODV video source sends only some video layers, the decoded frame rate is higher than using AODV, with which the entire video flow is transmitted. Consequently, the quality of the received video (in terms of PSNR) obtained with AQA-AODV also is better than the quality obtained when AODV is used.

The adaptive mechanisms of AQA-AODV provided a rapid response to the abrupt decrease of the available bandwidth; especially in the first scenario, when the route went from having two to four hops. Similarly, even though the mobility conditions in the second scenario affected the performance of the three evaluated protocols, the combination of the adaptive feedback scheme and the fast re-routing algorithm, allow AQA-AODV to minimize the impact of the mobility over the pauses or video gaps.

As discussed in the previous chapter, the conservative admission control of QADOV is not suitable for video streaming over highly dynamic networks as MANETs, despite it can handle certain levels of QoS and avoids network congestion.

On the other hand, the simulation study also shows a suitable integration of the developed framework SVCEval-RA and AQA-AODV, which constitutes a useful 
evaluation tool for performing network simulations that involve rate-adaptive video transmission using scalable video coding (SVC)

\subsection{Summary}

This chapter analyses the use of the proposed protocol AQA-AODV in the provision of rate-adaptive video services. In particular, a realistic and efficient system was created exploiting the layered scheme of SVC and the QoS mechanisms of AQA-AODV. Basically, the operation of the proposed solution consist on the adaptation of the bit rate, carried out by the video source, adding or removing SVC layers from the original video stream based on the estimation of the available bandwidth performed by AQA-AODV. For the evaluation of this rate-adaptive strategy, it was necessary to implement a new simulation framework, called SVCEval-RA. This framework has been developed in order to solve the lack of a video evaluation tool-set that supports simulation experiments that involve rate-adaptive video transmission of using the H.264/SVC standard. In particular, the SVCEval-RA framework works by generating two video traces from a real H.264/SVC encoded video. One trace is used to inform the traffic source about the composition of each SVC layer and another trace is used as input to the network simulator NS-2 in order to provide information about the packetization and the transmission of the video frames. During simulation, the video packet are assembled and delivered to the receiver through the simulated MANET. Moreover, during transmission the source can dynamically adjust its bit rate by filtering the packets belonging a specific SVC layer based on the estimation of the available bandwidth provided by the AQA-AODV protocol. Later, the output of the simulation is utilized to produce the received video stream. Latest version of SVCEVal-RA is available for free download at [108].

Finally, a simulation study over the SVCEval-RA platform was proposed. The aim of this experiment set was to evaluate the combine use of AQA-AODV and SVC coding for supporting adaptive video streaming. The obtained results demonstrate that the QoS mechanisms of AQA-AODV provides a suitable system for resource estimation and measurement. Hence, video application can know the current network status in order to transmit only the SVC layers that can be efficiently supported by network. Without this network-adaptive strategy, the video may not be adapted, causing congestion in the network and a large number of dropped packets, which is much worse than transmitting video using low data rate. Consequently, the quality of the delivered videos has been significantly better than using AODV or QAODV, both in static and in mobile scenarios. 


\section{Chapter 5}

\section{QoS-aware \\ gateway \\ discovery extension for AQA-AODV}

In most practical applications, ad hoc mobile devices demand the access to wired hosts located beyond the limits of the mobile ad hoc network, forming a heterogeneous wired cum wireless network. This interconnection requires a network device known as gateway. The gateway functions as a bridge between the ad hoc environment and the infrastructure-based networks, as Internet. In this chapter, it is described a gateway discovery mechanism implemented as an extension of the AQA-AODV routing protocol to improve interconnection performance between MANET and the Internet

\subsection{Interconnectivity in heterogeneous networks}

MANETs are generally conceived to operate as stand-alone networks, which means that data traffic will be restricted within the MANETs. All nodes in the MANET equally participate in the exchange of routing information, by running the same routing protocol. Most routing protocols for MANETs were originally designed for supporting communications within an autonomous MANET where no intervention of any centralised router exists. Therefore, a mobile ad hoc node cannot obtain the routing information beyond the scope of the MANET. To support communications between mobile ad hoc nodes and host devices in a wired network (e.g. Internet) routing protocols must be modified. The interoperability in heterogeneous networks, i.e. between ad hoc networks and other kinds of networks, such as cellular networks, infrastructure-based WLANs (Wireless Local Area Networks) and, especially, wired networks has an increasing attention. Basically, the communication between wireless ad hoc networks and infrastructure-based networks is established through network devices (Gateways) that understand not only the IP stack protocol, but also the MANET protocol suite (see Figure 5.1). Therefore, a gateway is an interface between MANET and the infrastructure-based networks, as Internet. 


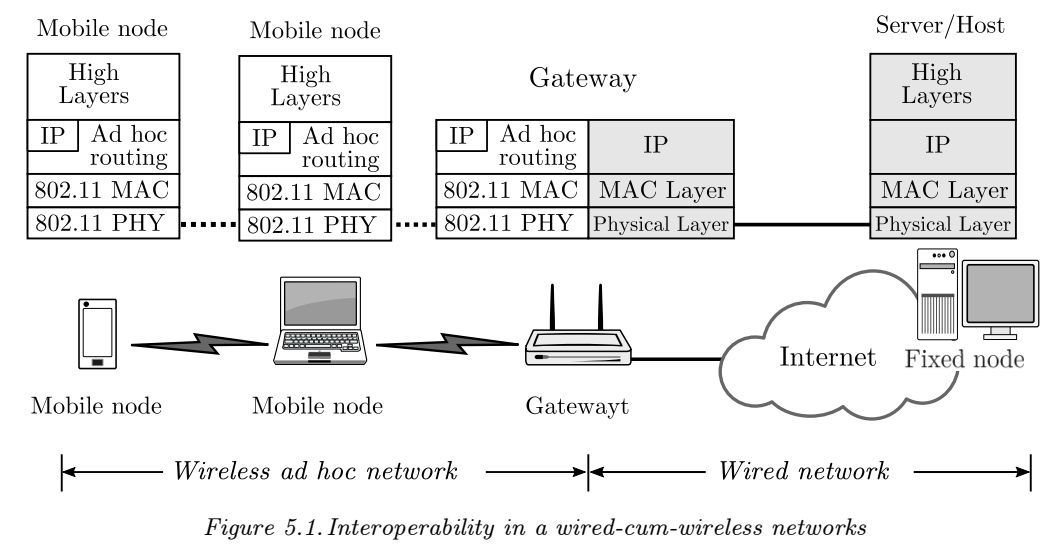

When a mobile node in an ad hoc network wants to establish a communication with a wired node, it has to discover the available gateways and select one of them. The gateway discovery process can be executed following a proactive or reactive method. In a proactive scheme, gateways periodically broadcast an advertisement message throughout ad hoc network. Each node that receives the advertisement message forwards the advertisement to other nodes until the message is flooded in the entire network. This scheme provides good connectivity and lower delay but high routing message overhead is incurred since the gateway advertisements are broadcast even if there is no such demand from the nodes in the MANET. On the other hand, in reactive schemes a mobile node broadcasts a gateway discovery message to discover gateways in the network. Once a gateway receives the discovery message, it unicasts a gateway advertisement message back to the requestor node. Although this scheme achieves the routing overhead, it may increase route discovery delay since mobile nodes have to send a gateway discovery message every time they need a gateway. Few solutions [109] [110] propose hybrid gateway discovery schemes where the dissemination of gateway advertisements is kept limited to a small range of n-hops (proactive area). Nodes outside the coverage area use a reactive scheme to find their gateways.

Three key elements must be considered in the hybrid gateway discovery scheme. The first element is the selection of best gateway when the requesting node receives multiple advertisement messages from gateways. Most of gateway selection schemes proposed only use the hop count metric to select a gateway [111] [112]. Therefore, in these schemes nodes always select the nearest gateway. However, although a route between a mobile node and a certain gateway has fewer hops than other routes, it may have a higher traffic load. The second elements to consider is related to the determination of the optimal proactive area. A large proactive area involves an increase in the routing overhead since routes must be maintained in a wider area. On the contrary, if proactive area is small there will be less maintenance overhead but more delay during the gateway discovery is experienced. The third issue is the adjustment of the frequency at which the advertisement messages are broadcast within the proactive area. In traditional hybrid gateway discovery scheme, the size of the proactive area and the frequency of the 
advertisement messages are set up statically and never updated. This leads to a rigid implementation of the hybrid scheme.

In this chapter is presented a new hybrid gateway discovery algorithm, which was integrated in the AQA-AODV protocol, for supporting the connectivity between MANETs and infrastructure-based networks. The gateway discovery algorithm includes available bandwidth as a metric during the gateway selection routine, which is a more suitable solution for providing multimedia communications. Moreover, the issues of the conventional hybrid scheme were solved adapting dynamically the size of the proactive area and the frequency of the advertisement messages.

\subsection{Related works}

During the last years, several approaches have been proposed for interconnecting wired and wireless networks. Most of the proposed solutions are based on the IETF Draft "Global Connectivity for IPv6 Mobile Ad-hoc Networks" suggested by Wakikawa et al. [113]. This work defines two different schemes for gateway discovery: periodic dissemination of gateway advertisement (GWADV) messages from the gateways and reactive broadcast of gateway solicitation (GWSOL) message from nodes. These reactive and proactive schemes are not dependent on any routing solution. However, this approach does not give any metric to select a gateway. Few solutions [111] [112] proposed a gateway selection method based on the number of physical hops to gateway. Nevertheless, this metric is not always optimal in the long term. The gateway selection proposed by Iqbal and Kabir [109] is based on three parameters: the interface queue size, the total number of neighbours of each node along the route and the hop count. They define a hybrid discovery method where gateways broadcast advertisement messages when they receive a gateway discovery message from a mobile node. The TTL (Time To Live) of the gateway advertisement message is set to a value equal to the distance of the gateway from the requesting node. Another hybrid approach is presented by Adédjouma et al. [114]. They extended the AODV routing protocol with a hybrid scheme for gateway discovery that exploits the opportunistic routing. With this technique, while a mobile node is in transit between two different gateways, it can temporally store messages before retransmitting. Zhang and $\mathrm{Hu}$ [110] apply biologically inspired metaphor to design a hybrid gateway discovery. Moreover, they propose a distributed gateway discovery based on the prediction model of mobility of nodes, which may require high processing power consumption and nodes have to wait longer period of time to select a path to a gateway. The work presented by Yuste et al. [115] propose a novel technique by which the messages that the gateway periodically generates are exclusively forwarded in the areas where the links are expected to remain stable.

However, the solutions discussed above do not perform a dynamic adaptation neither the size of the proactive area nor of the frequency of the advertisement messages. Zama et al. [116] and Triviño et al. [117] have studied the problem of the adjustment of the proactive range and the advertisement frequency, respectively. Their findings suggest and a dynamic adaptation of these parameters according to the networks conditions such as the mobility of nodes, the position and number of mobile nodes. Several 
adaptive strategies have been designed. For instance, Yuste et al. [118] proposed a fuzzy logic system to control the frequency of the advertisement message and an adaptation of the TTL value based on the maximal source coverage. Yan et al. [119] proposes a QoSbased gateway selection mechanism based on three QoS metrics: traffic load of gateway, path quality from mobile node to the gateway and hop count to the gateway.

\subsection{Proposed Gateway discovery process in AQA-AODV}

The implemented gateway discovery mechanism is an adapted algorithm from ideas suggested in the IETF Draft [113]. This document proposes implementing a hybrid gateway discovery in order to minimize the disadvantages of the proactive and reactive strategies. In contrast with the proactive method, overhead and network congestion are reduced since the $G W A D V$ messages of the hybrid approach are propagated only a limited number of hops away from the gateway (advertisement zone) and not along the entire network. On the other hand, hybrid method shows a better performance than the reactive approach, in terms of latency.

In the proposed hybrid discovery strategy, mobile nodes within a limited range (a certain number of hops away from the gateway) perform a proactive discovery while mobile nodes located outside this range use reactive gateway discovery (see Figure 5.2).

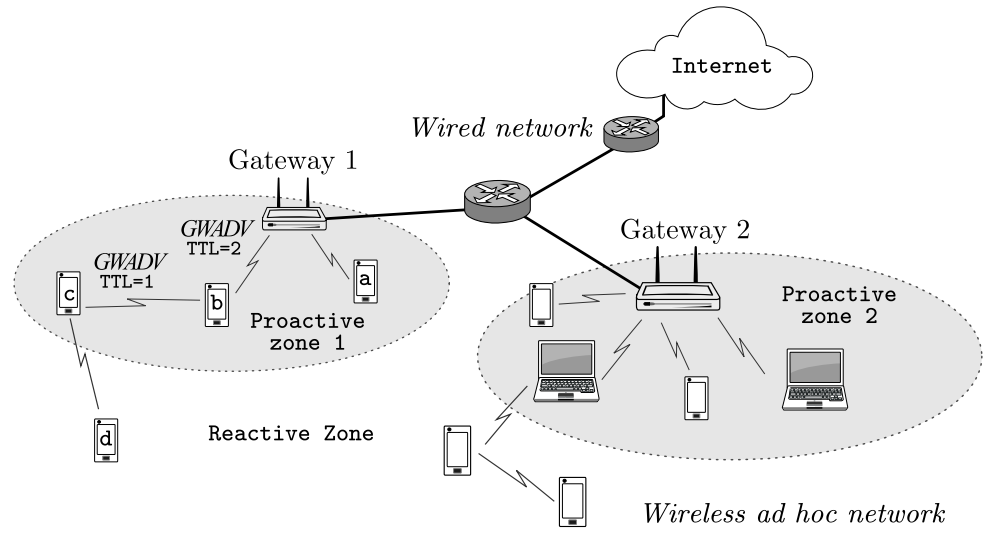

Figure 5.2. Hybrid gateway discovery

\subsubsection{Gateway discovery process}

In the proposed approach, gateways periodically broadcasts a GWADV (Gateway Advertisement) message with a certain $T T L$ value. The TTL parameter defines the range in which proactive discovery method is used (advertisement zone). The mobile nodes that receive the GWADV message update their routing table and then rebroadcast the message. For example, in Figure 5.2, gateway 1 broadcast a GWADV message with TTL equal to 2 . Consequently, mobile nodes " $a$ " and " $b$ " receive this message since they are within the transmission range of gateway 1 . On receiving the GWADV message, they decrement the TTL value from 2 to 1 and forward it. Mobile node " $c$ " receives the 
forwarded $G W A D V$ message. This mobile node again decrements the TTL value, which now becomes 0 . Therefore, the GWADV is not further forwarded and mobile node " $d$ " should use the reactive method for gateway discovery. The mobile nodes registered with gateway 2 follow a similar strategy.

When a mobile node in the MANET needs to establish a route to a fixed node (e.g. located in Internet), firstly it has to find a gateway. Therefore, mobile node looks for a default route (i.e. a route to a gateway) in its routing table. If mobile node finds a default route, it uses this route to transmit packets to the gateway. This fact indicates that mobile node is inside the proactive range. However, if the mobile node is not within the proactive area, it could not find a route to a gateway in its routing table. Consequently, it starts a gateway discovery process sending a Gateway Request message (GWREQ). If other mobile nodes receives this message, they rebroadcast it until the GWREQ message is received by a gateway (see Figure 5.3a). Once a gateway receives a GWREQ message, it responds sending back a unicast reply message, named GWREP (Gateway Reply), to the requesting node. A new field (named $B W$ ) in the GWREP message header was added to the conventional packet header. The $B W$ field is used to store the available bandwidth of the nodes along the route from a gateway to the requesting node. Once an intermediate node between gateway and the requesting node receives a GWREP message, it compares its local available bandwidth with the bandwidth indicated in the GWREP. If its local available bandwidth is lower, it replaces the value stored in the $B W$ field of the GWREP message, with the value of its available bandwidth. Otherwise, the node forwards the GWREP message (see Figure 5.3b). Consequently, this procedure will ensure that the requesting node knows the available bandwidth of the route to each gateway and select the best route towards Internet.

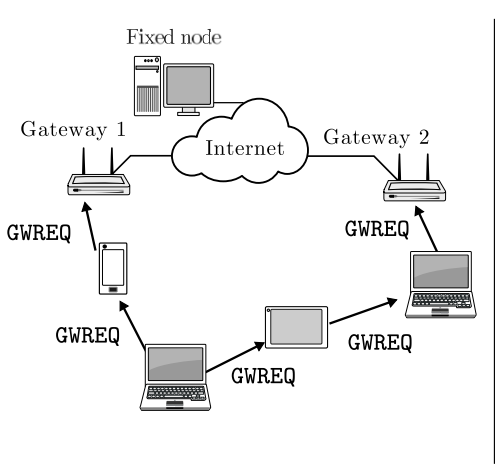

(a)

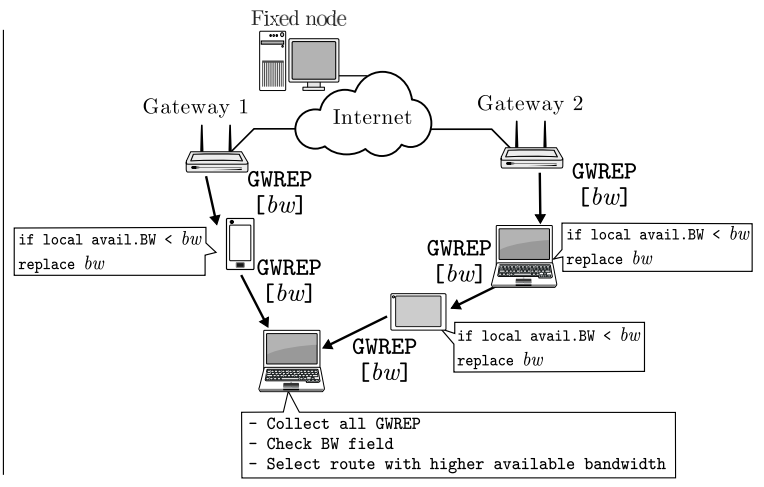

(b)

Figure 5.3. Message sequence in gateway selection procedure

GWREQ and GWREP messages were implemented in AQA-AODV by simply adding an $I$ flag to existing route request $(Q R R E Q)$ and route reply $(Q R R E P)$ packets in order to make the implementation easier. 


\subsection{1.a. Gateway advertisement adaptation}

It is important to note that there are two main issues in the design of a hybrid gateway discovery which may have a high impact in the overhead and the overall network performance [120] [116]. The first issue is how to determine the optimal proactive area (TTL value). If the proactive area is large (i.e. a high TTL value), more overhead is incurred in maintaining routes in a wider area. On the other hand, if proactive area is small, then less maintenance overhead is incurred but more delay during the gateway discovery is experienced.

The second issue is determining the time interval between two consecutive gateway advertisements (known as advertisement interval, $T_{a d v}$ ). A short advertisement interval allow mobile nodes to maintain an updated route to gateways. However, it can saturate the network with $G W A D V$ messages unnecessarily, increasing the protocol overhead both in bandwidth utilization and battery power consumption. On the other hand, a high advertisement interval could lead to the storage of out-of-date routing information in the mobile nodes. This fact implies that mobiles node have to start a reactive process, sending a GWREQ message, when they need to forward packets to the gateway. Therefore, an autonomous and dynamic algorithm to adapt the value of the advertisement interval is strongly recommended.

In the proposed discovery strategy, these two parameters are dynamically adapted depending on the networks conditions. With the aim of adapting the TTL of the GWADV message, it is proposed an adaptive approach based on the distribution of mobile nodes. In the proposed adaptive method, each gateway keeps a mobile node list to record the information of every active mobile node that has an active route to Internet established through it. The information includes mobile node IP address, numbers of hops to the gateway and lifetime (as shown Figure 5.4). This information is easily obtained from the IP header of data packets. Gateway can periodically query the mobile node list in order to calculate the average distance (in number of hops) of the mobile nodes registered in that list. This average distance will be the TTL value of the next GWADV message to be broadcast by the gateway. The average number of hops is an approximate indicator of how the mobile nodes are distributed around a gateway. If the average number of hops decreases means that only few mobile nodes are closed to gateway, then the TTL value should decrease to reduce the overhead by avoiding the dissemination of unnecessary GWADV messages. Otherwise, the TTL value should be increased to cover more mobile nodes and avoid the release of excessive GWREQ messages.

In addition, for the dynamic adaptation of the advertisement interval ( $\left.T_{a d v}\right)$ a modified version of the algorithm presented in [121] has been implemented. With this algorithm, the advertisement interval varies according to the network stability. If the mobiles nodes are near the gateway, routes can be assumed as more stable and therefore, the advertisement interval may be increased. If the nodes remain just a short period of time under the coverage of the gateway, most routes will probably be broken in the short term and GWADV message should be sent more frequently (advertisement interval must be decreased). The minimum value that can be assigned to the advertisement interval $\left(T_{a d v}\right)$ is 3 seconds, in accordance with the recommendations given in the standard [122]. 
And the maximum value that advertisement interval $\left(T_{a d v}\right)$ can reach is 30 seconds according to the outcomes of the study of Yuste et al. [115]. Between these two limits, the value of the advertisement interval is set according to a linear function, which is proportional to a parameter, called Network Stability Factor (NSF), such as shown in Figure 5.5.

\begin{tabular}{|c|c|c|c|}
\hline 1 & Address of mobile node 1 & Hop count & Life time \\
\hline 2 & Address of mobile node 2 & Hop count & Life time \\
$\vdots$ & $\vdots$ & $\vdots$ & $\vdots$ \\
\hline $\mathrm{n}$ & Address of mobile node $\mathrm{n}$ & Hop count & Life time \\
\hline
\end{tabular}

Figure 5.4. Format used for the list of active mobile nodes

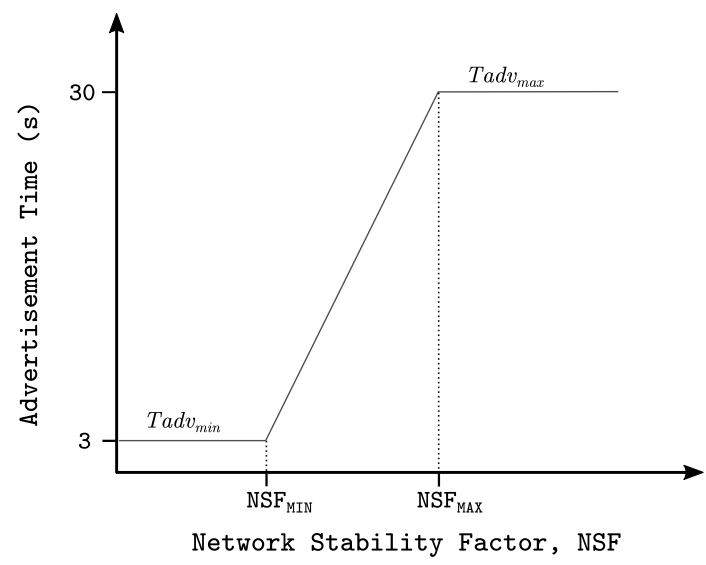

Figure 5.5. Relationship between NSF and the advertisement time

The estimation of NSF is performed by gateways based on the variation of the number of nodes that are under their coverage during two consecutive advertisement intervals. There are several methods for estimating the NSF parameter. In this thesis, a modified version of the method proposed by Trujillo et al. in [121] is used for calculating the NSF parameter, which can be defined as shown Equation (7).

$$
N S F=\frac{2 \times N_{r}-N_{n r}}{N_{G W A D V}(i)+N_{G W A D V}(i+1)}
$$

Where, $N_{G W A D V}(i)$ and $N_{G W A D V}(i+1)$ represent the number of GWADV messages received by gateway at intervals $i$-th and $(i+1) t h$, respectively. $N_{r}$ is the number of remaining nodes that, being under the coverage of the gateway at interval $i$ - $t h$, also are under the coverage of the gateway at interval $(i+1) t h . N_{n r}$ is the number of not remaining nodes, i.e. those nodes that are under the coverage of a Gateway in the $i$-th interval but not at 
interval $(i+1)-t h$. This information is easily collected by gateway analysing the GWADV messages forwarded by the mobiles nodes that are under its coverage.

Figure 5.6 illustrates an example of the estimation of the network stability factor. In Figure 5.6a nodes labelled as $a, b, c$ and $d$ are forwarding the GWADV messages to the gateway during the $i$-th interval. Similarly, in Figure 5.6b the nodes that are forwarding the $G W A D V$ messages during the (i+1)th interval are those labelled as $a, b, c, x$ and $y$.

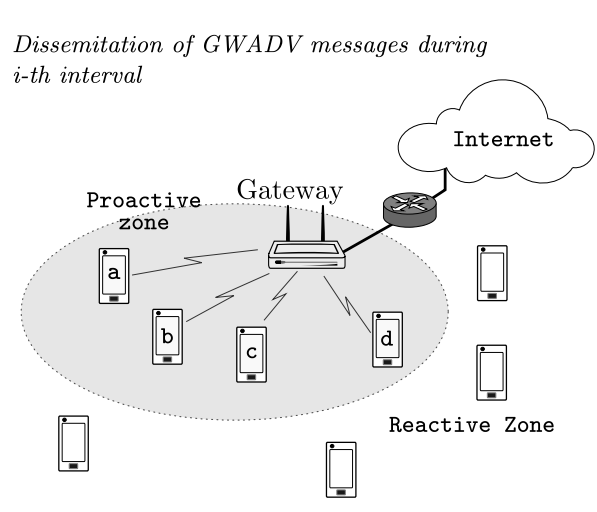

(a)

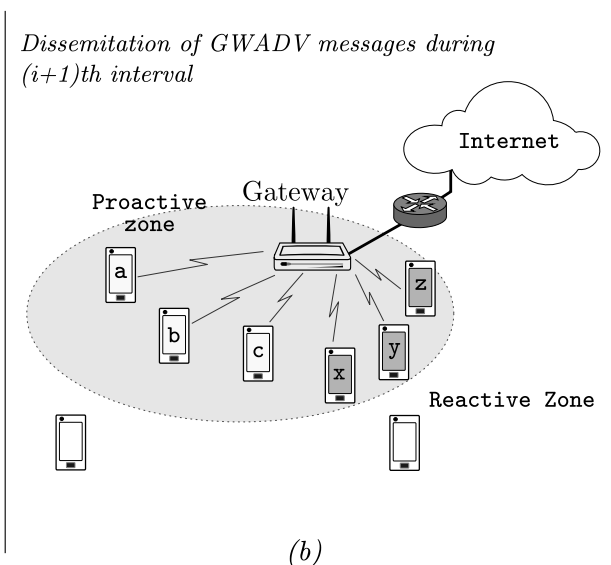

(b)

Figure 5.6. Example for NSF calculation

Table 5.1. Information for the calculation of the network stability factor

\begin{tabular}{c|c|c|c}
\hline \hline $\begin{array}{c}\text { Nodes forwarding } \\
\text { GWADV messages at } \\
\text { interval } \boldsymbol{i} \text {-th }\end{array}$ & $\begin{array}{c}\text { Nodes forwarding } \\
\text { GWADV messages at } \\
\text { interval }(\boldsymbol{i}+1)-t h\end{array}$ & $\begin{array}{c}\text { Remaining } \\
\text { Nodes }\end{array}$ & $\begin{array}{c}\text { Non- } \\
\text { remaining } \\
\text { nodes }\end{array}$ \\
\hline $\mathrm{a}, \mathrm{b}, \mathrm{c}, \mathrm{d}$ & $\mathrm{a}, \mathrm{b}, \mathrm{c}, \mathrm{x}, \mathrm{y}, \mathrm{z}$ & $\mathrm{a}, \mathrm{b}, \mathrm{c}$ & $\mathrm{d}, \mathrm{x}, \mathrm{y}, \mathrm{z}$ \\
\hline
\end{tabular}

Table 5.1 summarizes the information that can be extracted from the example of Figure 5.6. According to Table 5.1, the $N_{r}=3, N_{n r}=4, N_{G W A D V}(i)=4, N_{G W A D V}(i+1)=6$ and using Equation (7), the network stability factor is equal to 0.2 .

The maximum and minimum values for the NSF parameter are 1 and -1 , respectively. A stability factor of 1 represents a network with very low mobility while a value of -1 represents a network with very high mobility and low connectivity. In order to show how the NSF parameter characterizes the network stability, Figure 5.7 provides an example of the variation of $N S F$ versus the number of non-remaining nodes, $N_{n r}$. In this case, $N_{r}$ has been fixed to 15 and $N_{r n}$ varies between 0 and 30 .

As seen in Figure 5.7, when the number of non-remaining nodes increases, the stability factor decreases. This case occurs when nodes have a high mobility and they quickly arrive and leave the coverage areas of the gateways, diminishing the network stability. 
On the contrary, when the mobility of the nodes is low, there will be many remaining nodes and NSF may increase.

As mentioned above, the network stability factor (NSF) is used to calculate a new value of the advertisement interval $T_{a d v}$, following the function illustrated in Figure 5.5 and the values listed in Table 5.2. These values ensure that, when the mobility of the nodes is low (high connectivity) $T_{a d v}$ has a fixed value. And, when the mobility is high (low connectivity), $T_{a d v}$ will have the minimum value.

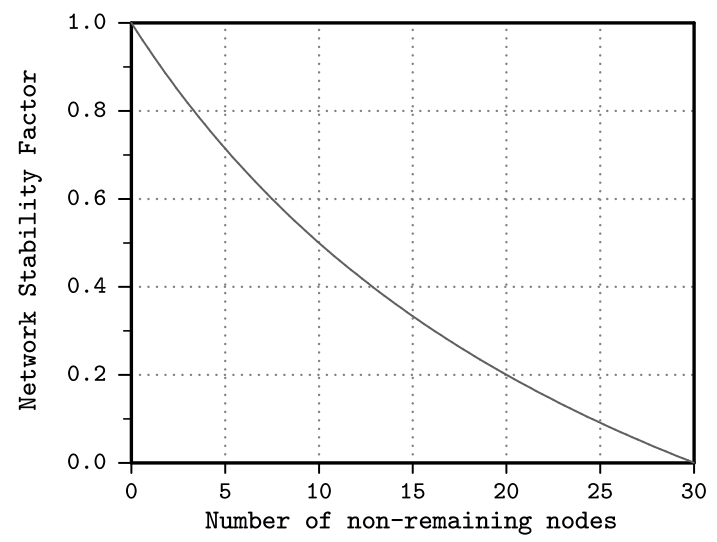

Figure 5.7. Variation of the Network Stability Factor

Table 5.2. Values for the estimation of the advertisement interval

\begin{tabular}{c|c|c|c}
\hline \hline $\boldsymbol{T a d}_{\text {MIN }}$ & $\boldsymbol{T a d v}_{\text {MAX }}$ & $\boldsymbol{N S F}_{\min }$ & $\boldsymbol{N S F}_{\text {max }}$ \\
\hline $3 \mathrm{~s}$ & $30 \mathrm{~s}$ & 0.2 & 0.8 \\
\hline
\end{tabular}

\subsubsection{Route establishment}

Once a mobile node selects a route to a gateway, it can initiate a route discovery process towards the fixed node (i.e. Internet host) using the conventional route discovery packets of AQA-AODV. Specifically, the mobile node sends a unicast QRREQ packet to the fixed node, informing its available bandwidth. This bandwidth value is the minimum value between the bandwidth informed by route during the gateway discovery process and its own estimation of the available bandwidth. When the fixed node receives the QRREQ packet, it configures its route to the gateway, adjusts its bit rate according to the available bandwidth and sends data packets to the requesting mobile node.

\subsection{Performance evaluation}

In this section, the proposed gateway discovery algorithm is analysed comparing it with other similar approaches under the same network conditions. For this evaluation, extensive simulations using the network simulator NS-2 were conducted. The proposed approach (hereinafter cited as AQA-Hybrid) was compared with the well-known 
reactive protocol (also known as AODV+) proposed by Hamidian [111] and the recent strategy (QGWS, QoS-based Gateway Selection) developed by Yan et al. [119].

The metrics used for the performance analysis of the proposed algorithms were:

1. Packet loss rate, which corresponds to the ratio between the number of lost packets and the total number of packets sent by the MANET nodes.

2. Average end-to-end delay, which is defined as the time elapsed since the emission of the data packet from the source until it reaches its final destination and.

3. Normalized routing overhead, that represents the ratio between the number of control packets and the number of received data packets.

\subsection{Simulation Environment}

The simulated scenario consists of 2 gateways, 2 fixed routers, 2 wired hosts and 30 mobile nodes randomly distributed over an area of 1000 × $300 \mathrm{~m}$ (Figure 5.8 illustrates a graphical representation of the simulated scenario). The mobile nodes move according to the random waypoint model with a variable speed from 2 to $10 \mathrm{~m} / \mathrm{s}$. The gateways are located at the $\mathrm{x}$ - and $\mathrm{y}$-coordinates $(100,100)$ and $(900,100)$. The wireless channel capacity for each mobile node is $2 \mathrm{Mb} / \mathrm{s}$, using the IEEE $802.11 \mathrm{~b}$ DCF MAC layer and a transmission range of $250 \mathrm{~m}$. The radio propagation model is Two Ray Ground and queue type is Drop Tail with maximum length of 50.

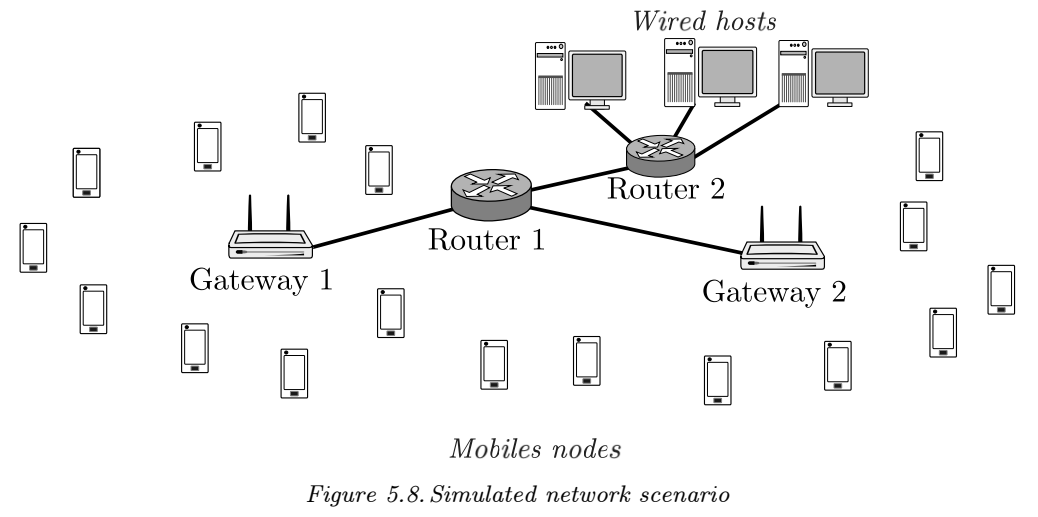

Two scenarios with different traffic load were tested. The first scenario has 10 traffic sources and the second one has 15 sources communicating with nodes in the wired network. All traffic flows are Constant Bit Rate (CBR) streams over UDP with a packet size of 512 bytes. The packet transmission is established between a random sourcedestination pair.

The simulation time was configured to 300 seconds and each data point shown in the figures is the average of 10 simulations with different random seed. In Table 5.3 are described some simulation parameters. 


\subsection{Results}

The results presented in Figure 5.9 shows the percentage of packet loss obtained for the three solutions. Results reveal that the differences between the proposed scheme and other approaches are very significant. Comparing Figure 5.9a and Figure 5.9b, it can be seen that with an increase in the number of sources, the performance of the AODV+ and QGWS approaches decreased. Whereas AQA-Hybrid maintained its percentage of lost packets below 11\% (about 2 and 3 times lower than QGWS and AODV+, respectively). The high packet loss of the AODV+ and QGWS approaches could be caused by network congestion in the mobile nodes. This congestion is generated when intermediate nodes between a mobile node and the gateway, cannot efficiently retransmit the packets due to they do not have enough available bandwidth. Connectivity losses could be an additional explanation for the high packet losses. However, because of the high density of nodes as well as the fact that there are two gateways in the network, it is more probable that there are always routes to gateways. Consequently, the packet losses due to connectivity losses are occasional. The drooped packet rate increases as speed increase because the link lifetimes decrease with the speed. Therefore, routes break easier and the transmission of the data packets through the broken routes will increment the losses.

Table 5.3. Simulation Parameters

\begin{tabular}{l|l}
\hline \hline \multicolumn{1}{c|}{ Parameter } & \multicolumn{1}{c}{ Value } \\
\hline Simulation area & $1000 \times 300 \mathrm{~m}$ \\
Wired link capacity & $10 \mathrm{Mbps}$ \\
Simulation time & $300 \mathrm{~s}$ \\
Mobility pattern & Maximum speed: varies from 2 to $10 \mathrm{~m} / \mathrm{s}$ \\
& Pause time: $10 \mathrm{~s}$ \\
& Scenario 1: 10 CBR sources \\
Data traffic & Scenario 2: 15 CBR sources \\
& Data rate 50 Kbps \\
Number of mobile nodes & Packet size 512 Bytes \\
& 30 \\
Advertisement interval & Adaptive from 3 to 30 s in AQA-Hybrid \\
& 5 s in QGWS and AODV+ approaches. \\
Size of proactive area & Adaptive size in AQA-Hybrid \\
& 3 hops in QGWS and AODV+. \\
\hline
\end{tabular}

As expected, the AODV+ proposal presents a high delay, which is significantly reduced using the hybrid approaches. Moreover, the delay associated to AQA-Hybrid is evidently lower than delay obtained by the other analysed approaches. Figure 5.10 shows that the average end-to-end delay of AQA-Hybrid is always below $0.12 \mathrm{~s}$ in both scenarios. There are similar trend in relation to the packet loss metric since as the number of sources increases, the difference in the performance of the three approaches also increases. Even though the traffic load increases, AQA-Hybrid remains a similar performance due to its adaptive scheme since the traffic sources can adapt its data rate to the available bandwidth avoiding network congestion. Consequently, the delay associated to the 
detection and repair of the link failures is also reduced. The values of delay and packet loss obtained with AODV+ and QGWS allow us to infer that these proposals are not suitable for supporting multimedia traffic.

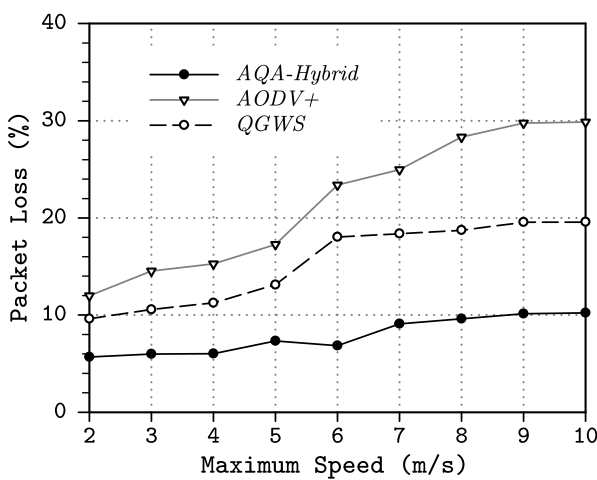

(a)

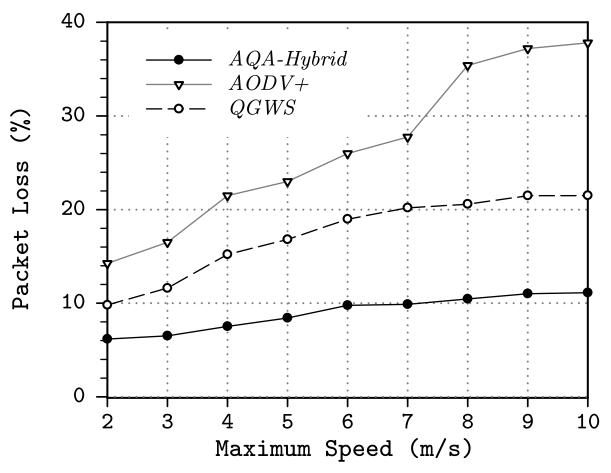

(b)

Figure 5.9. Packet Loss with variable node speed in both scenarios: (a) 10 sources and (b) 15 sources

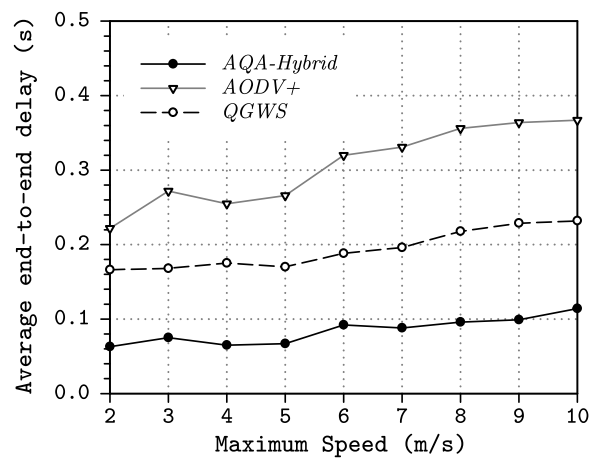

(a)

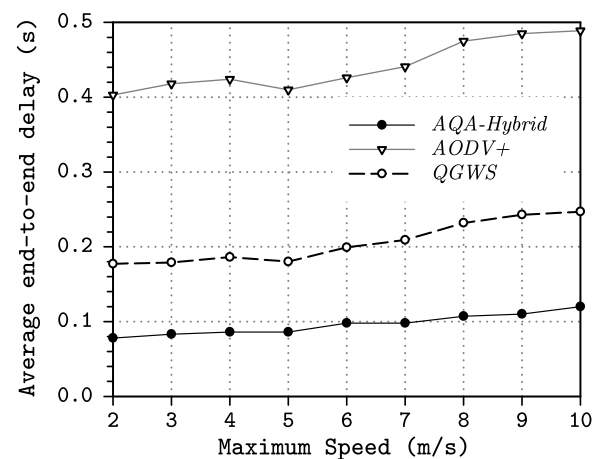

(b)

Figure 5.10. Delay as a function of the node speed in both scenarios: (a) 10 sources and (b) 15 sources

Regarding routing overhead, the results are presented in Figure 5.11. As a consequence of the stability of the routes, in the scenario with 10 sources and for lower speed values the AODV+ approach obtains the best performance in terms of routing overhead since stable routes prevent the execution of the reactive gateway discovery process. However, as the node speed increases, the number of gateway request packets in the reactive scheme of AODV+ also increases, due to an increment of the broken routes. Thus, for higher speed values the hybrid approaches (QGWS and AQA-Hybrid) present a better performance in both scenarios. When the number of sources is increased (Figure 5.11b), the differences between the approaches also increase because more sources require to perform the reactive gateway discovery through the whole network. On the other hand, under all mobility levels in both scenarios AQA-Hybrid overall outperforms QGWS due to the dynamic adjustment of the frequency of gateway advertisement messages as well 
as the adaptation of the TTL value of the advertisement messages according to the node mobility, which allow the gateways to reduce the number of control packets.

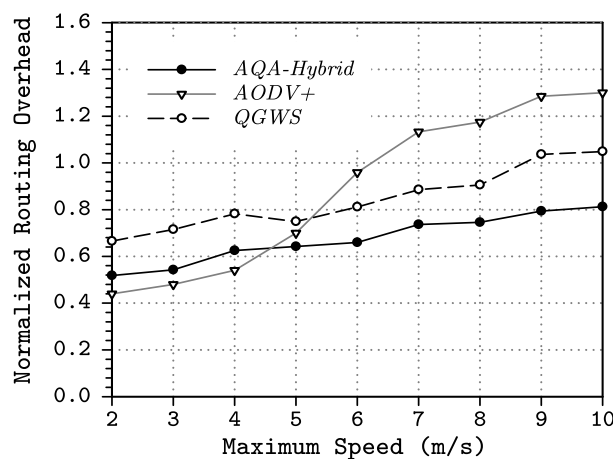

(a)

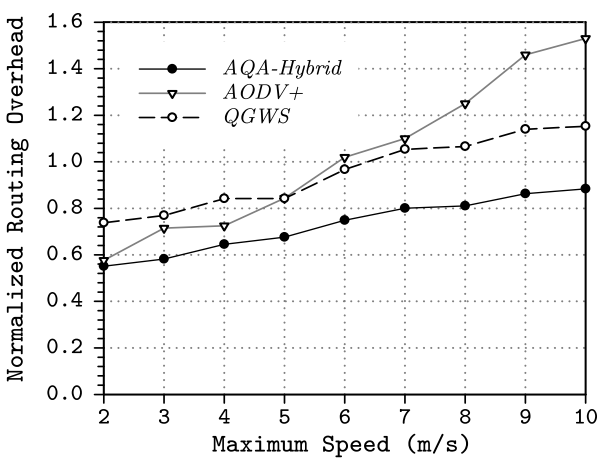

(b)

Figure 5.11. Routing overhead with variable node speed in both scenarios: (a) 10 sources and (b) 15 sources

\subsubsection{Conclusions}

The gateway discovery algorithm proposed in this chapter not only enables AQA-AODV to establish connections to infrastructure-based networks but also it provides an efficient QoS-aware solution to exchange video content between MANETs and Internet. Moreover, with the expected application of MANETs in the newer wireless network architectures, such as smart cities and the Internet of things, the gateway discovery mechanisms become an essential feature for the routing protocols over MANETs. Compared with other hybrid approaches, the proposed gateway discovery method incorporates the available bandwidth as a metric during the gateway selection. Moreover, it dynamically adapts the size of the proactive area and the frequency of the advertisement messages in order to make more efficient the dissemination of control packets.

\subsection{Summary}

A new hybrid gateway discovery strategy has been proposed in this chapter as an extension of the AQA-AODV protocol in order to improve the interoperability of MANETs and infrastructure-based networks. As described in Chapter 3, AQA-AODV is a routing protocol suitable for purely ad hoc environments that includes a mechanism for estimation of the available bandwidth and a feedback scheme to provide information to the application about the available bandwidth. With this information, traffic source may dynamically adapt its data rate to network conditions. With the hybrid discovery procedure added to AQA-AODV, it is not only able to establish routes according to the QoS requirements of the requesting node in a MANET, but also is able to establish communications with a host in a wired network through gateways. 
The main characteristics of the gateway discovery algorithm proposed in this thesis can be summarized as follows. The first one is its hybrid design where the proactive and reactive methods are combined. Consequently, gateways maintain routing information sending advertisement messages to mobile nodes inside a limited range (proactive area). Those nodes located outside that area must execute a reactive gateway discovery process. The second element is the incorporation of the available bandwidth in the selection of the best gateway. Finally, the third main feature is the dynamic adaptation of some operational parameters, such as the sending frequency of the advertisement messages and the size of the area where these advertisement messages are disseminated.

A performance evaluation of the proposed gateway discovery scheme was conducted in several networks scenarios together with other similar approaches. The simulation results show that the most substantial improvement of the proposed solution is the ability of reduces network congestion (i.e. packet loss and delay) without increasing routing overhead. 


\section{References}

[1] Wiley: Mobile Ad Hoc Networking: The Cutting Edge Directions, 2nd Edition - Stefano Basagni, Marco Conti, Silvia Giordano, et al. 2013.

[2] M. K. Gulati and K. Kumar, 'QoS routing protocols for mobile ad hoc networks: a survey', Int. J. Wirel. Mob. Comput., vol. 5, no. 2, pp. 107-118, Jan. 2012.

[3] S. Zheng, L. Li, and Y. Li, 'A QoS Routing Protocol for Mobile Ad Hoc Networks Based on Multipath', J. Netw., vol. 7, no. 4, 2012.

[4] L. Ullah Khan, S. A. Mahmud, M. H. Zafar, G. M. Khan, and H. S. Al-Raweshidy, 'M-AODV: Modified Ad Hoc On-demand distance vector routing scheme', in 2014 9th International Symposium on Communication Systems, Networks Digital Signal Processing (CSNDSP), 2014, pp. 18-22.

[5] J. Zhou, L. Liu, Y. Deng, and S. Huang, 'A QoS Routing Protocol with Bandwidth Allocation in Multichannel Ad Hoc Networks', Wirel. Pers. Commun., vol. 75, no. 1, pp. 273-291, Mar. 2014.

[6] M. Sanabani, R. Alsaqour, and S. Kurkushi, 'A reverse and enhanced aodv routing protocol for manets', ARPN J. Eng. Appl. Sci., vol. 9, no. 2, pp. 153-159, 2014.

[7] T. Coelho, A. Costa, J. Macedo, and M. J. Nicolau, 'Improving Stability in QoS Routing for AdHoc Networks', in Ad Hoc Networks, N. Mitton, A. Gallais, M. E. Kantarci, and S. Papavassiliou, Eds. Springer International Publishing, 2014, pp. 121-133.

[8] Y. Xu, J. D. Deng, M. Nowostawski, and M. K. Purvis, 'Optimized routing for video streaming in multi-hop wireless networks using analytical capacity estimation', J. Comput. Syst. Sci., vol. 81, no. 1, pp. 145-157, Feb. 2015.

[9] S. Pudlewski, N. Cen, Z. Guan, and T. Melodia, 'Video Transmission Over Lossy Wireless Networks: A Cross-Layer Perspective', IEEE J. Sel. Top. Signal Process., vol. 9, no. 1, pp. 6-21, Feb. 2015.

[10] S. Guo, M. Derakhshani, M. H. Falaki, U. Ismail, R. Luk, E. A. Oliver, S. U. Rahman, A. Seth, M. A. Zaharia, and S. Keshav, 'Design and implementation of the KioskNet system', Comput. Netw., vol. 55, no. 1, pp. 264-281, Jan. 2011.

[11] IIT-Madras, 'SARI (Sustainable Access in Rural India) Project'. [Online]. Available: http://edev.media.mit.edu/SARI/. [Accessed: 04-Apr-2015].

[12] Avri Doria, 'Providing connectivity to the Saami nomadic community'. [Online]. Available: http://pure.ltu.se/portal/en/publications/providing-connectivity-to-the-saami-nomadiccommunity(08269d80-ec0d-11db-88eb-000ea68e967b).html. [Accessed: 04-Apr-2015].

[13] G. Theraulaz and E. Bonabeau, 'A Brief History of Stigmergy', Artif. Life, vol. 5, no. 2, pp. 97116, Apr. 1999. 
[14] IEEE Standard, 'IEEE -1609 WG - Dedicated Short Range Communication Working Group'. [Online]. Available: https://standards.ieee.org/develop/wg/1609_WG.html. [Accessed: 04Apr-2015].

[15] M. Chen, S. Gonzalez, A. Vasilakos, H. Cao, and V. C. Leung, 'Body Area Networks: A Survey', Mob Netw Appl, vol. 16, no. 2, pp. 171-193, Apr. 2011.

[16] S. Ditlea, 'The PC goes ready-to-wear', IEEE Spectr., vol. 37, no. 10, pp. 34-39, Oct. 2000.

[17] E. Jovanov and A. Milenkovic, 'Body Area Networks for Ubiquitous Healthcare Applications: Opportunities and Challenges', J. Med. Syst., vol. 35, no. 5, pp. 1245-1254, Feb. 2011.

[18] E. Jovanov, A. Milenkovic, C. Otto, and P. C. de Groen, 'A wireless body area network of intelligent motion sensors for computer assisted physical rehabilitation', J. NeuroEngineering Rehabil., vol. 2, no. 1, pp. 1-10, Mar. 2005.

[19] C. Otto, A. Milenković, C. Sanders, and E. Jovanov, 'System Architecture of a Wireless Body Area Sensor Network for Ubiquitous Health Monitoring', J Mob Multimed, vol. 1, no. 4, pp. 307-326, Jan. 2005.

[20] P. J. Bennett, K. Soga, I. Wassell, P. Fidler, K. Abe, Y. Kobayashi, and M. Vanicek, 'Wireless sensor networks for underground railway applications: case studies in Prague and London', Smart Struct. Syst., vol. 6, no. 5-6, pp. 619-639, 2010.

[21] F. Stajano, N. Hoult, I. Wassell, P. Bennett, C. Middleton, and K. Soga, 'Smart bridges, smart tunnels: Transforming wireless sensor networks from research prototypes into robust engineering infrastructure', Ad Hoc Netw., vol. 8, no. 8, pp. 872-888, 2010.

[22] H. Schaffers, N. Komninos, M. Pallot, B. Trousse, M. Nilsson, and A. Oliveira, 'Smart Cities and the Future Internet: Towards Cooperation Frameworks for Open Innovation', in The Future Internet, J. Domingue, A. Galis, A. Gavras, T. Zahariadis, D. Lambert, F. Cleary, P. Daras, S. Krco, H. Müller, M.-S. Li, H. Schaffers, V. Lotz, F. Alvarez, B. Stiller, S. Karnouskos, S. Avessta, and M. Nilsson, Eds. Springer Berlin Heidelberg, 2011, pp. 431-446.

[23] S. Alawadhi, A. Aldama-Nalda, H. Chourabi, J. R. Gil-Garcia, S. Leung, S. Mellouli, T. Nam, T. A. Pardo, H. J. Scholl, and S. Walker, 'Building Understanding of Smart City Initiatives', in Electronic Government, H. J. Scholl, M. Janssen, M. A. Wimmer, C. E. Moe, and L. S. Flak, Eds. Springer Berlin Heidelberg, 2012, pp. 40-53.

[24] 'iCity Project -Works for the quality of citizen's live', 2012. [Online]. Available: http://www.icityproject.eu/. [Accessed: 06-Apr-2015].

[25] 'MOBINCITY Project - Smart mobility in smart city', 2013. [Online]. Available: http://www.mobincity.eu/. [Accessed: 06-Apr-2015].

[26] G. S. Khekare and A. V. Sakhare, 'A smart city framework for intelligent traffic system using VANET', in Automation, Computing, Communication, Control and Compressed Sensing (iMac4s), 2013 International Multi-Conference on, 2013, pp. 302-305.

[27] V. Kostakos, T. Ojala, and T. Juntunen, 'Traffic in the Smart City: Exploring City-Wide Sensing for Traffic Control Center Augmentation', Internet Comput. IEEE, vol. 17, no. 6, pp. 22-29, 2013.

[28] L. Atzori, A. Iera, and G. Morabito, 'The internet of things: A survey', Comput. Netw., vol. 54, no. 15, pp. 2787-2805, 2010.

[29] E. Borgia, 'The Internet of Things vision: Key features, applications and open issues', Comput. Commun., vol. 54, pp. 1-31, Dec. 2014.

[30] G. Bianchi, 'Performance analysis of the IEEE 802.11 distributed coordination function', IEEE J. Sel. Areas Commun., vol. 18, no. 3, pp. 535-547, Mar. 2000.

[31] P. Gupta and P. R. Kumar, 'The capacity of wireless networks', IEEE Trans. Inf. Theory, vol. 46, no. 2, pp. 388-404, Mar. 2000. 
[32] K. Sanzgiri, I. D. Chakeres, and E. M. Belding-Royer, 'Determining intra-flow contention along multihop paths in wireless networks', in Proceedings of the First International Conference on Broadband Networks, 2004, pp. 611-620.

[33] J. L. Bordim, A. V. Barbosa, M. F. Caetano, and P. S. Barreto, 'IEEE802.11b/g Standard: Theoretical Maximum Throughput', in 2010 First International Conference on Networking and Computing (ICNC), 2010, pp. 197-201.

[34] J. Jun, P. Peddabachagari, and M. Sichitiu, 'Theoretical maximum throughput of IEEE 802.11 and its applications', in Second IEEE International Symposium on Network Computing and Applications, 2003. NCA 2003, 2003, pp. 249-256.

[35] T. C. Thang, H. T. Le, H. X. Nguyen, A. T. Pham, J. W. Kang, and Y. M. Ro, 'Adaptive video streaming over HTTP with dynamic resource estimation', J. Commun. Netw., vol. 15, no. 6, pp. 635-644, Dec. 2013.

[36] K. Evensen, T. Kupka, H. Riiser, P. Ni, R. Eg, C. Griwodz, and P. al Halvorsen, 'Adaptive Media Streaming to Mobile Devices: Challenges, Enhancements, and Recommendations', Adv. Multimed., vol. 2014, pp. 10:10-10:10, Jan. 2014.

[37] C. Timmerer, C. Griwodz, A. C. Begen, T. Stockhammer, and B. Girod, 'Adaptive Media Streaming.', IEEE J. Sel. Areas Commun., vol. 32, no. 4, pp. 681-683, 2014.

[38] Ce Zhu and Yuenan Li, Advanced Video Communications over Wireless Networks. .

[39] ITU-T, 'Rec. H.264 \& ISO/IEC 14496-10 AVC. Advanced Video Coding for Generic Audiovisual Services'. 2013.

[40] S. Xiang, L. Cai, and J. Pan, 'Adaptive scalable video streaming in wireless networks', in Proceedings of the 3rd Multimedia Systems Conference, New York, NY, USA, 2012, pp. 167172.

[41] E. Yaacoub, F. Filali, and A. Abu-Dayya, 'QoE Enhancement of SVC Video Streaming over Vehicular Networks Using Cooperative LTE/802.11p Communications', IEEE J. Sel. Top. Signal Process., vol. PP, no. 99, pp. 1-1, 2014.

[42] K. Birkos, C. Tselios, T. Dagiuklas, and S. Kotsopoulos, 'Peer selection and scheduling of H. 264 SVC video over wireless networks', in Wireless Communications and Networking Conference (WCNC), 2013 IEEE, 2013, pp. 1633-1638.

[43] Video Traces Research Group, 'YUV Sequences'. [Online]. Available: http://trace.eas.asu.edu/yuv/index.html. [Accessed: 06-Jan-2015].

[44] C.-H. Ke, C.-K. Shieh, W.-S. Hwang, and A. Ziviani, 'An Evaluation Framework for More Realistic Simulations of MPEG Video Transmission.', J. Inf. Sci. Eng., vol. 24, no. 2, pp. 425440, 2008.

[45] Z. Wang, W. Wang, Y. Xia, Z. Wan, J. Wang, L. Li, and C. Cai, 'Visual Quality Assessment after Network Transmission Incorporating NS2 and Evalvid', Sci. World J., vol. 2014, p. e267403, Apr. 2014.

[46] C. Perkins, E. Belding-Royer, and S. Das, 'Ad hoc On-Demand Distance Vector (AODV) Routing, RFC 3561'. Network Working Group. Internet Engineering Task Force (IETF), 2003.

[47] T. Clausen and P. Jacquet, 'Optimized Link State Routing Protocol (OLSR), RFC 3626'. Network Working Group. Internet Engineering Task Force (IETF), 2003.

[48] D. Johnson, Y. Hu, and D. Maltz, 'The Dynamic Source Routing Protocol (DSR) for Mobile Ad Hoc Networks for IPv4 RFC'. Network Working Group. Internet Engineering Task Force (IETF), 2007.

[49] C. Perkins and P. Bhagwat, 'Highly Dynamic Destination-Sequenced Distance-Vector Routing (DSDV) for Mobile Computers', in Proceedings of the Conference on Communications Architectures, Protocols and Applications, New York, NY, USA, 1994, pp. 234-244. 
[50] C. Perkins, S. Ratliff, and J. Dowdell, 'Dynamic MANET On-demand (AODVv2) Routing (IETF Internet Draft - 05)'. Internet Engineering Task Force, 2014.

[51] P. Samar, M. R. Pearlman, and Z. J. Haas, 'Independent Zone Routing: An Adaptive Hybrid Routing Framework for Ad Hoc Wireless Networks', IEEEACM Trans Netw, vol. 12, no. 4, pp. 595-608, Aug. 2004.

[52] J. Wang, E. Osagie, P. Thulasiraman, and R. K. Thulasiram, 'HOPNET: A hybrid ant colony optimization routing algorithm for mobile ad hoc network', Ad Hoc Netw., vol. 7, no. 4, pp. 690-705, Jun. 2009.

[53] P. Arce Vila, 'Hierarchical routing and cross-layer mechanisms for improving video streaming quality of service over mobile wireless ad hoc networks', Editorial Universitat Politècnica de València, 2014.

[54] K. Xu, X. Hong, and M. Gerla, 'Landmark routing in ad hoc networks with mobile backbones', J. Parallel Distrib. Comput., vol. 63, no. 2, pp. 110-122, Feb. 2003.

[55] C.-C. Chiang, H.-K. Wu, W. Liu, and M. Gerla, 'Routing in clustered multihop, mobile wireless networks with fading channel', in IEEE Singapore International Conference on Networks (SICON '97), Singapore, 1997, vol. 97, pp. 197-211.

[56] M. Tarique and K. E. Tepe, 'Minimum energy hierarchical dynamic source routing for Mobile Ad Hoc Networks', Ad Hoc Netw., vol. 7, no. 6, pp. 1125-1135, Aug. 2009.

[57] V. Rishiwal, M. Yadav, S. K. Bajapai, and S. Verma, 'Power aware routing in ad hoc wireless networks', J. Comput. Sci. Technol., vol. vol. 9, no. 2, 2009.

[58] Y. Ahn, Y. Zhao, U. He, and J. Choi, 'Efficient Ad Hoc Routing Technique for Connecting Geographically Distributed Heterogeneous Networks', in Internet of Things, Y. Wang and X. Zhang, Eds. Springer Berlin Heidelberg, 2012, pp. 9-16.

[59] C.-H. Chou, K.-F. Ssu, and H. C. Jiau, 'Dynamic route maintenance for geographic forwarding in mobile ad hoc networks', Comput. Netw., vol. 52, no. 2, pp. 418-431, Feb. 2008.

[60] V. C. Giruka and M. Singhal, 'A self-healing On-demand Geographic Path Routing Protocol for mobile ad-hoc networks', Ad Hoc Netw., vol. 5, no. 7, pp. 1113-1128, Sep. 2007.

[61] C. Gui and P. Mohapatra, 'Hierarchical multicast techniques and scalability in mobile Ad Hoc networks', Ad Hoc Netw., vol. 4, no. 5, pp. 586-606, Sep. 2006.

[62] L. Sanna and L. Atzori, 'Group multicast routing problem: A genetic algorithms based approach', Comput. Netw., vol. 51, no. 14, pp. 3989-4004, Oct. 2007.

[63] C. Liu, M. Yarvis, W. S. Conner, and X. Guo, 'Guaranteed on-demand discovery of nodedisjoint paths in ad hoc networks', Comput. Commun., vol. 30, no. 14-15, pp. 2917-2930, Oct. 2007.

[64] L. Reddeppa Reddy and S. V. Raghavan, 'SMORT: Scalable multipath on-demand routing for mobile ad hoc networks', Ad Hoc Netw., vol. 5, no. 2, pp. 162-188, Mar. 2007.

[65] S. Y. Han, B. Shin, and D. Lee, 'An application-driven path discovery mechanism for MANET routing protocols', in Communications (ICC), 2014 IEEE International Conference on, 2014, pp. 2822-2827.

[66] X. Zhen and Y. Wenzhong, 'Bandwidth-aware routing for TDMA-based mobile ad hoc networks', in International Conference on Information Networking (ICOIN), Bangkok, Thailand, 2013, pp. 637-642.

[67] C. Perkins and E. Belding-Royer, 'Quality of Service for Ad hoc On-Demand Distance Vector Routing (IETF Internet Draft)'. Mobile Ad Hoc Networking Working Group. Internet Engineering Task Force (IETF), 2004.

[68] R. Mu and W. Zhang, 'QAODV: Improved security routing protocol of AODV', J. Netw., vol. 8, no. 10, pp. 2400-2405, 2013. 
[69] L. Liu, L. Zhu, L. Lin, and Q. Wu, 'Improvement of AODV Routing Protocol with QoS Support in Wireless Mesh Networks', Phys. Procedia, vol. 25, pp. 1133-1140, 2012.

[70] L. Chen and W. B. Heinzelman, 'QoS-aware routing based on bandwidth estimation for mobile ad hoc networks', IEEE J. Sel. Areas Commun., vol. 23, no. 3, pp. 561 - 572, 2005.

[71] V. Kumar and G. Ilanchezhiapandian, 'Enhancement of AODV Routing protocol to provide QOS for MANET', in Proceedings of the National Conference on Emerging Trends in Computing Science, 2010.

[72] A. Moussaoui and A. Boukeream, 'A survey of routing protocols based on link-stability in mobile ad hoc networks', J. Netw. Comput. Appl., vol. 47, pp. 1-10, Jan. 2015.

[73] Q. Xue and A. Ganz, 'Ad hoc QoS on-demand routing (AQOR) in mobile ad hoc networks', J. Parallel Distrib. Comput., vol. 63, no. 2, pp. 154-165, 2003.

[74] R. de Renesse, V. Friderikos, and H. Aghvami, 'Towards Providing Adaptive Quality of Service in Mobile Ad-Hoc Networks', in IEEE 63rd Vehicular Technology Conference, Melbourne, Australia, 2006, vol. 2, pp. 518-522.

[75] H. Huang, H. Yin, J. Zhang, and F. Qian, 'Delay-oriented power-aware routing in wireless ad hoc networks', Trans. Emerg. Telecommun. Technol., 2013.

[76] Y. Ping and W. Ying, 'A revised AODV protocol with QoS for Mobile Ad hoc Network', in IEEE International Conference on Computer Science and Information Technology, 2009, pp. 241 -244 .

[77] G. I. Ivascu, S. Pierre, and A. Quintero, 'QoS routing with traffic distribution in mobile ad hoc networks', Comput. Commun., vol. 32, no. 2, pp. 305-316, 2009.

[78] Y. Zhang and T. A. Gulliver, 'Quality of service for ad hoc on-demand distance vector routing', in IEEE International Conference on Wireless And Mobile Computing, Networking And Communications, Canada, 2005, vol. 3, pp. $192-196$.

[79] N. Sarma and S. Nandi, 'Route Stability Based QoS Routing in Mobile Ad Hoc Networks', Wirel. Pers. Commun., vol. 54, no. 1, pp. 203-224, 2010.

[80] H. Liu and L. Cheng, 'Available Bandwidth Estimation Strategy Based on the Network Allocation Vector', J. Netw., vol. 7, no. 12, pp. 2089-2095, Dec. 2012.

[81] C. Sarr, C. Chaudet, G. Chelius, and I. G. Lassous, 'A node-based available bandwidth evaluation in IEEE 802.11 ad hoc networks', in Proceedings of the 11th International Conference on Parallel and Distributed Systems, 2005, vol. 2, pp. 68-72.

[82] Haitao Zhao, Jibo Wei, Shan Wang, and Yong Xi, 'Available Bandwidth Estimation and Prediction in Ad hoc Networks', in Mobile Ad-Hoc Networks: Protocol Design, X. Wang, Ed. InTech, 2011, pp. 61-84.

[83] A. Munaretto and M. Fonseca, 'Routing and quality of service support for mobile ad hoc networks', Comput. Netw., vol. 51, no. 11, pp. 3142-3156, Aug. 2007.

[84] S. H. Shah, K. Chen, and K. Nahrstedt, 'Dynamic bandwidth management in single-hop ad hoc wireless networks', Mob. Netw. Appl., vol. 10, no. 1-2, pp. 199-217, 2005.

[85] 'The Network Simulator (NS-2)'. [Online]. Available: http://www.isi.edu/nsnam/ns. [Accessed: 06-Feb-2015].

[86] H. Schwarz, D. Marpe, and T. Wiegand, 'Overview of the Scalable Video Coding Extension of the H.264/AVC Standard', IEEE Trans. Circuits Syst. Video Technol., vol. 17, no. 9, pp. 11031120, 2007.

[87] I. Unanue, I. Urteaga, R. Husemann, J. Del Ser, V. Roesler, A. Rodríguez, and P. Sánchez, 'A Tutorial on H. 264/SVC Scalable Video Coding and its Tradeoff between Quality, Coding Efficiency and Performance', Recent Adv. Video Coding, pp. 1-24, 2011. 
[88] Y. Wang and M. Claypool, 'RealTracer-Tools for Measuring the Performance of RealVideo on the Internet', Multimed. Tools Appl., vol. 27, no. 3, pp. 411-430, Dec. 2005.

[89] R. Karki, T. Seenivasan, M. Claypool, and R. Kinicki, 'Performance Analysis of Home Streaming Video Using Orb', in Proceedings of the 20th International Workshop on Network and Operating Systems Support for Digital Audio and Video, New York, NY, USA, 2010, pp. 111-116.

[90] M. Dai, Y. Zhang, and D. Loguinov, 'A unified traffic model for MPEG-4 and H. 264 video traces', IEEE Trans. Multimed., vol. 11, no. 5, pp. 1010-1023, 2009.

[91] M. T. Ivrlač, L. U. Choi, E. Steinbach, and J. A. Nossek, 'Models and analysis of streaming video transmission over wireless fading channels', Signal Process. Image Commun., vol. 24, no. 8, pp. 651-665, Sep. 2009.

[92] F. Espina, D. Morato, M. Izal, and E. Magaña, 'Analytical model for MPEG video frame loss rates and playback interruptions on packet networks', Multimed. Tools Appl., vol. 72, no. 1, pp. 361-383, Sep. 2014.

[93] S. Tanwir and H. Perros, 'A Survey of VBR Video Traffic Models', IEEE Commun. Surv. Tutor., vol. 15, no. 4, pp. 1778-1802, Fourth 2013.

[94] S. Tanwir and H. G. Perros, VBR Video Traffic Models. John Wiley \& Sons, 2014.

[95] D. Fiems, B. Steyaert, and H. Bruneel, 'A genetic approach to Markovian characterisation of H.264 scalable video', Multimed. Tools Appl., vol. 58, no. 1, pp. 125-146, 2012.

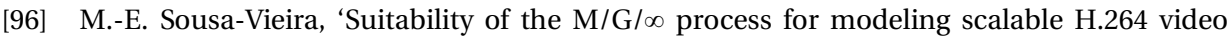
traffic', in Analytical and Stochastic Modeling Techniques and Applications, Springer, 2011, pp. 149-158.

[97] G. Van der Auwera, P. T. David, M. Reisslein, and L. J. Karam, 'Traffic and quality characterization of the H. 264/AVC scalable video coding extension', Adv. Multimed., vol. 2008, no. 2, p. 1, 2008.

[98] J. Klaue, B. Rathke, and A. Wolisz, 'Evalvid-A framework for video transmission and quality evaluation', in Computer Performance Evaluation. Modelling Techniques and Tools, Springer, 2003, pp. 255-272.

[99] A. Lie and J. Klaue, 'Evalvid-RA: trace driven simulation of rate adaptive MPEG-4 VBR video', Multimed. Syst. J., vol. 14, no. 1, pp. 33-50, 2008.

[100] A. Detti, G. Bianchi, C. Pisa, F. S. Proto, P. Loreti, W. Kellerer, S. Thakolsri, and J. Widmer, 'SVEF: an open-source experimental evaluation framework for H.264 scalable video streaming', in IEEE Symposium on Computers and Communications, 2009, pp. 36-41.

[101] T. A. Le and H. Nguyen, 'End-to-end transmission of scalable video contents: performance evaluation over EvalSVC-a new open-source evaluation platform', Multimed. Tools Appl., vol. 72, no. 2, pp. 1239-1256, Sep. 2014.

[102] C.-H. Ke, 'myEvalSVC-an Integrated Simulation Framework for Evaluation of H. 264/SVC Transmission', KSII Trans. Internet Inf. Syst. TIIS, vol. 6, no. 1, pp. 377-392, 2012.

[103] Moving Pictures Experts Group and ITU-T Video Coding Experts Group, 'H. 264/SVC reference software (JSVM 9.19.14) and Manual'. Moving Pictures Experts Group and ITU-T Video Coding Experts Group, 2011.

[104] H. Sohn, H. Yoo, W. De Neve, C. S. Kim, and Y. M. Ro, 'Full-Reference Video Quality Metric for Fully Scalable and Mobile SVC Content', IEEE Trans. Broadcast., vol. 56, no. 3, pp. 269280, Sep. 2010.

[105] S. Akhshabi, A. C. Begen, and C. Dovrolis, 'An experimental evaluation of rate-adaptation algorithms in adaptive streaming over HTTP', in Proceedings of the second annual ACM conference on Multimedia systems, 2011, pp. 157-168. 
[106] 'YUV video repository'. [Online]. Available: ftp://ftp.tnt.unihannover.de/pub/svc/testsequences/. [Accessed: 10-Jan-2013].

[107] Xiph.Org Foundation, 'Video Test Media. DerfCollection', Video Test Media. Derf Collection. [Online]. Available: https://media.xiph.org/video/derf/.

[108] W. Castellanos, 'SVCEval-RA - An Evaluation Framework for Adaptive Scalable Video Streaming', SourceForge Project, 18-Aug-2014. [Online]. Available: http://sourceforge.net/projects/svceval-ra/. [Accessed: 14-Jan-2015].

[109] S. M. Asif-Iqbal and M. H. Kabir, 'Hybrid Scheme for Discovering and Selecting Internet Gateway in Mobile Ad Hoc Network', Int. J. Wirel. Mob. Netw., vol. 3, no. 4, pp. 83-101, Aug. 2011.

[110] G. Zhang and J. Hu, 'Adaptive Distributed Gateway Discovery with Swarm Intelligence in Hybrid Wireless Networks', Int. J. Future Gener. Commun. Netw., vol. 7, no. 5, pp. 137-150, Oct. 2014.

[111] A. Hamidian, 'Supporting Internet access and quality of service in distributed wireless ad hoc networks', Lund University, 2009.

[112] Y. He, Y. Ahn, and J. Choi, 'Gateway-Based Modified AODV for MANET and Internet Interconnection', in Proceedings of the Fourth International Conference on Signal and Image Processing 2012 (ICSIP 2012), 2013, pp. 179-192.

[113] R. Wakikawa, J. T. Malinen, C. E. Perkins, A. Nilsson, and A. J. Tuominen, 'Global connectivity for IPv6 Mobile Ad Hoc Networks. Internet Draft (work in progress)'. Internet Engineering Task Force, 2006.

[114] S. A. Adédjouma, P. A. Chokki, and T. K. Dagba, 'An Extended AODV Protocol to Support Mobility in Hybrid Networks', 2013.

[115] A. J. Yuste, A. Triviño, F. D. Trujillo, and E. Casilari, 'Improved Scheme for Adaptive Gateway Discovery in Hybrid MANET', in 2010 IEEE 30th International Conference on Distributed Computing Systems Workshops (ICDCSW), 2010, pp. 270-275.

[116] R. U. Zaman, A. V. Reddy, and others, 'A Survey of Adaptive Gateway Discovery Mechanisms in Heterogeneous Networks', Int. J. Comput. Netw. Inf. Secur. IJCNIS, vol. 5, no. 7, p. 34, 2013.

[117] A. Triviño, B. Ruiz-Villalobos, E. Casilari, and A. J. Yuste-Delgado, 'Study on the Need for Adaptive Gateway Discovery in MANETs', in Proceedings of the 2009 International Conference on Wireless Communications and Mobile Computing: Connecting the World Wirelessly, New York, NY, USA, 2009, pp. 1091-1095.

[118] A. J. Yuste, A. Triviño, and E. Casilari, 'Type-2 fuzzy decision support system to optimise MANET integration into infrastructure-based wireless systems', Expert Syst. Appl., vol. 40, no. 7, pp. 2552-2567, Jun. 2013.

[119] Y. Yan, L. Ci, Z. Wang, and W. He, 'QoS-based gateway selection in MANET with Internet connectivity', in 2013 15th International Conference on Advanced Communication Technology (ICACT), 2013, pp. 195-199.

[120] S. Majumder and Asaduzzaman, 'A hybrid gateway discovery method for mobile ad hoc networks', in 2014 International Conference on Informatics, Electronics Vision (ICIEV), 2014, pp. 1-6.

[121] F. D. Trujillo, A. Triviño, E. Casilari, A. Diaz-Estrella, and A. J. Yuste, 'A stability approach to improve MANET-internet connection', in 23rd International Symposium on Computer and Information Sciences, 2008. ISCIS '08, 2008, pp. 1-6.

[122] T. Narten, W. A. Simpson, E. Nordmark, and H. Soliman, 'Neighbor Discovery for IP version 6 (IPv6) - IETF RFC 4861'. 2007. 



\section{Appendix A}

\section{List of Publications}

The publications generated from the research work of this thesis can be listed as follows.

\section{Journal Papers}

1. P. Acelas, P. Arce, J. C. Guerri, and W. Castellanos, "Evaluation of the MDC and FEC over the quality of service and quality of experience for video distribution in ad hoc networks", Multimedia Tools and Applications, vol. 68, no. 3, pp. 969989, 2014.

\section{Papers under evaluation}

1. W. Castellanos, J. C. Guerri and P Arce, "A QoS-aware routing protocol with adaptive feedback scheme for video streaming for mobile networks", Computer Communications 2015, http://www.journals.elsevier.com/computercommunications/, submitted, under revision.

2. W. Castellanos, J. C. Guerri and P Arce, "SVCEval-RA: An Evaluation Framework for Adaptive Scalable Video Streaming”, Multimedia Tools and Applications, 2015, submitted, under evaluation.

3. W. Castellanos, J. C. Guerri and P Arce, "Available bandwidth estimation for adaptive video streaming in mobile ad hoc networks", Journal of Applied Research and Technology, 2015, http://www.elsevier.es/en-revista-journal-ofapplied-research-and-81, submitted, under evaluation.

4. W. Castellanos, J. C. Guerri and P Arce, "Performance Evaluation of Scalable Video Streaming in Mobile Ad hoc Networks", IEEE Latin America Transactions, 2015, submitted, under evaluation.

5. W. Castellanos, J. C. Guerri, M. Chacón. “A hybrid gateway discovery algorithm for supporting QoS communications in heterogeneous networks", Revista de la 
Facultad de Ingeniería, Universidad de Antioquia, 2015, submitted, under evaluation.

6. W. Castellanos, J. C. Guerri, M. Chacón, "Improving video transmission over heterogeneous networks", Revista de la Facultad de Ingeniería, Universidad Central de Venezuela, 2015, submitted, under evaluation.

\section{Conference papers}

1. W. Castellanos, P. Arce, P. Acelas, and J. C. Guerri, "Route recovery algorithm for QoS-aware routing in MANETs" in Proc. of the ICST Conference on Mobile Lightweight Wireless Systems (MOBILIGHT '11), Bilbao, Spain, pp. 81-93, May 2011.

2. P. Acelas, P. Arce, W. Castellanos, and J. C. Guerri, "Estudio sobre el Efecto de la Codificación de Vídeo por Multidescripción en Redes MANET" presented at XV Simposium Nacional de la Unión Científica Internacional de Radio (URSI '10), Bilbao, Spain, Sep. 2010.

3. W. Castellanos, P. Acelas, P. Arce, and J. C. Guerri, "AQA-AODV: QoS-aware $A O D V$ protocol with adaptive feedback scheme", presented at the ICST Conference on Heterogeneous Networking for Quality, Reliability, Security and Robustness (QSHINE '09), Las Palmas, Spain, Nov. 2009.

\section{Book Chapters}

1. J. C. Guerri, P. Arce, P. Acelas, W. E. Castellanos, and F. Fraile, "Routing and Coding Enhancements to Improve QoS of Video Transmissions in Future Ad Hoc Networks", Multimedia Services and Streaming for Mobile Devices: Challenges and Innovations, IGI Global, pp. 244-261, 2012.

Additionally, during the elaboration of the thesis, the author realized a research stay at the Biomechanical Institute of Valencia where has participated in the following projects:

- ISTOPPFALLS: ICT based System to Predict and Prevent Falls (2011-2014), FP7ICT-2011-7-287361

- HARKEN: Heart and respiration in-car embedded nonintrusive sensors (20112014), FP7-SME-2011-286265

- PPUSIM: Pay per Use for a Sustainable Intelligent Mobility, (2012-2014), Instituto de la Mediana y Pequeña Industria Valenciana. 